\title{
ASYMPTOTIC BEHAVIOR OF NONLINEAR ELLIPTIC SYSTEMS ON VARYING DOMAINS*
}

\author{
JUAN CASADO DIAZ ${ }^{\dagger}$ AND ADRIANA GARRONI ${ }^{\ddagger}$
}

\begin{abstract}
We consider a monotone operator of the form $A u=-\operatorname{div}(a(x, D u))$, with $\Omega \subseteq \mathbf{R}^{n}$ and $a: \Omega \times \mathbf{M}^{M \times N} \rightarrow \mathbf{M}^{M \times N}$, acting on $W_{0}^{1, p}\left(\Omega, \mathbf{R}^{M}\right)$. For every sequence $\left(\Omega_{h}\right)$ of open subsets of $\Omega$ and for every $f \in W^{-1, p^{\prime}}\left(\Omega, \mathbf{R}^{M}\right), 1 / p+1 / p^{\prime}=1$, we study the asymptotic behavior, as $h \rightarrow+\infty$, of the solutions $u_{h} \in W_{0}^{1}\left(\Omega_{h}, \mathbf{R}^{M}\right)$ of the systems $A u_{h}=f$ in $W^{-1, p^{\prime}}\left(\Omega_{h}, \mathbf{R}^{M}\right)$, and we determine the general form of the limit problem.
\end{abstract}

Key words. homogenization, perforated domains, Dirichlet systems

AMS subject classifications. 35B27, 35B40

PII. S0036141097329627

1. Introduction. In this paper we study the asymptotic behavior of the solutions of elliptic nonlinear systems, of $M$ equations and $N$ variables, on varying domains with Dirichlet boundary conditions. Namely, let $\Omega$ be a bounded open subset of $\mathbf{R}^{N}$ and let $1<p<+\infty$. We regard $A$ as a vector monotone operator defined from $W^{1, p}\left(\Omega, \mathbf{R}^{M}\right)$ to $W^{-1, p^{\prime}}\left(\Omega, \mathbf{R}^{M}\right)$, mapping $u \in W^{1, p}\left(\Omega, \mathbf{R}^{M}\right)$ in $A u=-\operatorname{div}(a(x, D u)) \in W^{-1, p^{\prime}}\left(\Omega, \mathbf{R}^{M}\right)$. The function $a: \Omega \times \mathbf{M}^{M \times N} \mapsto \mathbf{M}^{M \times N}$ is a Carathéodory function which satisfies the standard assumptions of strong monotonicity and Hölder continuity (see conditions (i)-(iv) in section 5). Given an arbitrary sequence of open subsets $\Omega_{n}$ of $\Omega$ and given an arbitrary $f \in W^{-1, p^{\prime}}\left(\Omega, \mathbf{R}^{M}\right)$, we consider the solutions $u_{n}$ of the following systems with Dirichlet boundary condition

$$
u_{n} \in W_{0}^{1, p}\left(\Omega_{n}, \mathbf{R}^{M}\right), \quad A u_{n}=f \quad \text { in } \Omega_{n} .
$$

We set $u_{n}=0$ in $\Omega \backslash \Omega_{n}$ and regard the sequence $\left(u_{n}\right)$ as a sequence in $W_{0}^{1, p}\left(\Omega, \mathbf{R}^{M}\right)$. Our results describe the asymptotic behavior of $\left(u_{n}\right)$ as $n \rightarrow \infty$ and characterize the limit function as the solution of a variational "limit problem."

The main result of this paper is given by the following compactness theorem.

THEOREM 1.1. Let $\Omega_{n}$ be an arbitrary sequence of open subsets of $\Omega$. Then there exist a subsequence of $\Omega_{n}$, still denoted by $\Omega_{n}$, a measure $\mu$ in the class $\mathcal{M}_{0}^{p}(\Omega)$ of positive Borel measures not charging set of p-capacity zero, and a vector function $F: \Omega \times \mathbf{R}^{M} \rightarrow \mathbf{R}^{M}$, such that for every $f \in W^{-1, p^{\prime}}\left(\Omega, \mathbf{R}^{M}\right)$ the sequence $\left(u_{n}\right)$ of solutions of problems (1.1) converges weakly in $W_{0}^{1, p}\left(\Omega, \mathbf{R}^{M}\right)$ to the solution $u$ of the variational problem

$$
\left\{\begin{array}{l}
u \in W_{0}^{1, p}\left(\Omega, \mathbf{R}^{M}\right) \cap L_{\mu}^{p}\left(\Omega, \mathbf{R}^{M}\right) \\
\int_{\Omega} a(x, D u) D v d x+\int_{\Omega} F(x, u) v d \mu=\langle f, v\rangle \\
\forall v \in W_{0}^{1, p}\left(\Omega, \mathbf{R}^{M}\right) \cap L_{\mu}^{p}\left(\Omega, \mathbf{R}^{M}\right) .
\end{array}\right.
$$

${ }^{*}$ Received by the editors November 5, 1997; accepted for publication (in revised form) September 9, 1998; published electronically February 24, 2000.

http://www.siam.org/journals/sima/31-3/32962.html

†Departamento de ecuaciones differenciales, C. Tarfia Sin, 41012 Sevilla, Spain (jcasado@ numer.us.es).

‡Dipartimento di Matematica, Università "La Sapienza," Piazzale A. Moro 2, 00185 Roma, Italy (garroni@mat.uniroma1.it). 
By $L_{\mu}^{p}\left(\Omega, \mathbf{R}^{M}\right)$ we denote the standard $L^{p}$ spaces with respect to the measure $\mu$. Note that in this general case the usual "extra term" is given by $\int_{\Omega} F(x, u) v d \mu$.

The problem considered in the present paper has been studied, under various degree of generality, by many authors, with several approaches and in different frameworks. Most of the known results are given under assumptions involving the geometry or the capacity of the closed sets $\Omega \backslash \Omega_{n}$, which in general imply that the measure $\mu$ in the limit problem is a Radon measure (see, for instance, [20], [22], and [7] for the linear case, and [24], [25], [26], [27], [21], and [3], for monotone operators).

The class $\mathcal{M}_{0}^{p}(\Omega)$ described above also includes measures which take the value $+\infty$ on large families of sets; in this way, Dirichlet problems in subdomains of $\Omega$ can be written in the form (1.2) for a suitable choice of $\mu$. Indeed, it is easy to see that, if $E$ is a closed subset of $\Omega$ and the measure $\mu$ is defined by

$$
\mu(B)= \begin{cases}0 & \text { if } C_{p}(B \cap E)=0 \\ +\infty & \text { otherwise }\end{cases}
$$

for every Borel subset $B$ of $\Omega$, where $C_{p}$ denotes the $p$-capacity, then problem (1.2) is equivalent to

$$
u \in W_{0}^{1, p}\left(\Omega \backslash E, \mathbf{R}^{M}\right), \quad A u=f \quad \text { in } \Omega \backslash E .
$$

In view of the latter remark, the compactness result above will be proved in a more general formulation (see Theorem 6.4) for a sequence of problems of the type

$$
\left\{\begin{array}{l}
u_{n} \in W_{0}^{1, p}\left(\Omega, \mathbf{R}^{M}\right) \cap L_{\mu_{n}}^{p}\left(\Omega, \mathbf{R}^{M}\right), \\
\int_{\Omega} a\left(x, D u_{n}\right) D v d x+\int_{\Omega} F_{n}\left(x, u_{n}\right) v d \mu_{n}=\langle f, v\rangle \\
\forall v \in W_{0}^{1, p}\left(\Omega, \mathbf{R}^{M}\right) \cap L_{\mu_{n}}^{p}\left(\Omega, \mathbf{R}^{M}\right),
\end{array}\right.
$$

which for a suitable choice of $\left(\mu_{n}\right)$ in $\mathcal{M}_{0}^{p}(\Omega)$ reduce to (1.1), and includes also Schrödinger systems with positive oscillating potentials. A further motivation for the study of problem (1.4) is given by the recent applications to a relaxed formulation of some optimal design problems (see, for instance, [2]).

The compactness result in the setting of (1.4) was first proved for the scalar case $M=1$, using $\Gamma$-convergence techniques, in [13] and [14] when $p=2$ and $A$ is a symmetric linear elliptic operator, and in [10] if $A$ is $p-1$ homogeneous, under the assumption that it is the subdifferential of a convex functional. These results were generalized using Tartar's energy method in [11] for the general scalar linear case, and subsequently for the nonlinear case under an assumption of homogeneity of order $p-1$ for the operator $A$ (see [15] and [16]). In these cases the extra term which appears in the limit problem is proved to be of the form $\int_{\Omega}|u|^{p-2} u v, d \mu$. The case of systems is much less investigated. Previous results have been obtained only in the framework of linear symmetric elliptic operators in [18]. Further reference on this subject can be found in the book [9] and in the papers [11] and [16], which contain a wide bibliography.

Our result provides a description of the limiting behavior of sequences of Dirichlet boundary value problems not only for monotone operators of Leray-Lions type, but also covering the case of systems related to linear possibly nonsymmetric operators or nonlinear homogeneous operators, which were not included in previous results. The proof follows the lines of [3], where Theorem 1.1 is obtained in the scalar case, under 
some additional assumptions on the sequence $\left(\Omega_{n}\right)$ which imply in particular that the measure $\mu$ in the limit problem is bounded. The idea of the proof is essentially to compare our sequence of problems with a sequence of model problems for which the behavior is known (for instance scalar problems with the $p$-Laplace operator).

In section 2 we recall some preliminary results and notation and in section 3 we state some known results in the study of the asymptotic behavior of scalar problems with the $p$-Laplace operator.

Section 4 is dedicated to a careful study of the behavior of a sequence of "correctors" for the $p$-Laplace operator, as introduced in [16]. In section 5 we state the problem and we prove, following the line of [1] and [16], that a sequence of solutions of problems (1.4) which converges weakly in $W^{1, p}\left(\Omega, \mathbf{R}^{M}\right)$ converges also strongly in $W^{1, r}\left(\Omega, \mathbf{R}^{M}\right)$ for every $r<p$ (see Proposition 5.4 ). In section 6 we prove the compactness result. In section 7 we prove a correctors result, in the general context of nonlinear monotone vector operators. Indeed, the sequence of gradients $\left(D u_{n}\right)$ of solutions of problems (1.1) converges to $D u$ a priori only weakly in $L^{p}$ by Theorem 1.1. Hence to obtain a strong convergence it is necessary to add a further sequence which depends only on the limit function $u$. The construction of such a sequence is provided by Theorem 7.1 and is new also in the case of linear systems. For previous correctors results, see, e.g., [7], [11], [3]. Section 8 is devoted to the analysis of some special cases. In particular we obtain a simpler form for the extra term and for the correctors in the linear case and in the homogeneous case, in agreement with the previous scalar results. The structure of the extra term is proved to depend only on the asymptotic behavior of the function $a(x, \xi)$ for $\xi \rightarrow \infty$ (see section 9 ). In the last section our result is applied also to the treatment of asymptotic problems in a class of pseudomonotone operators. The extension to the general pseudomonotone operators for the scalar case can be found in [5]. Throughout the paper we treat in detail only the case $p \geq 2$. The case $1 \leq p<2$ can be treated in a similar way, after proper modification on the growth and coerciveness hypotheses for the operator $A$. The changes in the proofs can easily be performed using Proposition 3.2 of [17].

2. Notation and preliminaries. Let $N$ and $M$ be two positive integers, $N \geq 2$; by $\mathbf{M}^{M \times N}$ we denote the space of $M \times N$ real matrices.

Let $\Omega$ be a bounded open subset of $\mathbf{R}^{N}$. We denote by $W_{0}^{1, p}\left(\Omega, \mathbf{R}^{M}\right)$ and $W^{1, p}\left(\Omega, \mathbf{R}^{M}\right), 1<p<+\infty$, the usual Sobolev spaces (of $\mathbf{R}^{M}$-valued functions) and by $W^{-1, p^{\prime}}\left(\Omega, \mathbf{R}^{M}\right), 1 / p^{\prime}+1 / p=1$, the dual of $W_{0}^{1, p}\left(\Omega, \mathbf{R}^{M}\right)$. By $W_{c}^{1, p}\left(\Omega, \mathbf{R}^{M}\right)$ and $W_{\text {loc }}^{1, p}\left(\Omega, \mathbf{R}^{M}\right)$ we denote respectively the space of all functions in $W^{1, p}\left(\Omega, \mathbf{R}^{M}\right)$ with compact support in $\Omega$ and the space of all functions which belong to $W^{1, p}\left(U, \mathbf{R}^{M}\right)$ for every open set $U \subset \subset \Omega$. When $p=2$ we adopt the standard notation $H^{1}\left(\Omega, \mathbf{R}^{M}\right)$, $H_{0}^{1}\left(\Omega, \mathbf{R}^{M}\right)$, and $H^{-1}\left(\Omega, \mathbf{R}^{M}\right)$.

By $L_{\mu}^{p}\left(\Omega, \mathbf{R}^{M}\right), 1 \leq p \leq+\infty$, we denote the usual Lebesgue space with respect to the measure $\mu$. If $\mu$ is the Lebesgue measure, we use the standard notation $L^{p}\left(\Omega, \mathbf{R}^{M}\right)$.

When we consider space of scalar functions $(M=1)$, we omit $\mathbf{R}^{M}$ in the notations above.

Let $u \in W^{1, p}(\Omega)$ and $k \in \mathbf{R}$. By $T_{k} u$ we shall denote the truncation at the level $k$ which is the function in $W^{1, p}(\Omega)$ defined by $T_{k} u=(-k) \wedge u \vee k$.

Let $A$ be an open set in $\mathbf{R}^{N}, u: A \rightarrow \mathbf{R}^{M}$ and $a, b \in \mathbf{R}$; we shall denote by $\{a \leq|u| \leq b\}_{A}$ the set of all $x \in A$ such that $a \leq|u(x)| \leq b$. When $A=\Omega$ we shall omit $\Omega$ in the notation above.

We shall use $o_{m, n}$ (respectively, $o_{n}$ ) to denote a sequence of real numbers such that $\lim _{m \rightarrow \infty} \lim _{n \rightarrow \infty} o_{m, n}=0$ (respectively, $\lim _{n \rightarrow \infty} o_{n}=0$ ). 
If $E \subseteq \Omega$, the (harmonic) p-capacity of $E$ in $\Omega$, denoted by $C_{p}(E)$, is defined as the infimum of

$$
\int_{\Omega}|D u|^{p} d x
$$

over the set of all functions $u \in W_{0}^{1, p}(\Omega)$ such that $u \geq 1$ almost everywhere (a.e.) in a neighborhood of $E$.

We say that a property $\mathcal{P}(x)$ holds p-quasi everywhere (abbreviated as p-q.e.) in a set $E$ if it holds for all $x \in E$ except for a subset $N$ of $E$ of $p$-capacity zero. The expression $\mu$-almost everywhere (abbreviated as $\mu$-a.e.) refers, as usual, to the analogous property for a Borel measure $\mu$.

A function $u: \Omega \rightarrow \mathbf{R}^{M}$ is said to be p-quasi continuous if for every $\varepsilon>0$ there exists a set $A \subseteq \Omega$, with $C_{p}(A)<\varepsilon$, such that the restriction of $u$ to $\Omega \backslash A$ is continuous.

It is well known that every $u \in W^{1, p}\left(\Omega, \mathbf{R}^{M}\right)$ has a $p$-quasi continuous representative, which is uniquely defined up to a set of $p$-capacity zero. In the following we shall always identify $u$ with its $p$-quasi continuous representative, so that the pointwise values of a function $u \in W^{1, p}\left(\Omega, \mathbf{R}^{M}\right)$ are defined $p$-q.e. in $\Omega$.

A subset $A$ of $\Omega$ is said to be p-quasi open in $\Omega$ if for every $\varepsilon>0$ there exists an open subset $A_{\varepsilon}$ of $\Omega$, with $C_{p}\left(A_{\varepsilon}\right)<\varepsilon$, such that $A \cup A_{\varepsilon}$ is open. It is easy to see that if a function $u: \Omega \rightarrow \mathbf{R}$ is $p$-quasi continuous, then the set $\{u>c\}$ is $p$-quasi open for every $c \in \mathbf{R}$. For all these properties of $p$-quasi continuous representatives of Sobolev functions we refer to [28, Chapter 3].

By a nonnegative Borel measure in $\Omega$ we mean a countably additive set function defined in the Borel $\sigma$-field of $\Omega$ and with values in $[0,+\infty]$. By a nonnegative Radon measure in $\Omega$ we mean a nonnegative Borel measure which is finite on every compact subset of $\Omega$. We shall always identify a nonnegative Borel measure with its completion.

We say that a Radon measure $\nu$ on $\Omega$ belongs to $W^{-1, p^{\prime}}(\Omega)$ if there exists $f \in$ $W^{-1, p^{\prime}}(\Omega)$ such that

$$
\langle f, \varphi\rangle=\int_{\Omega} \varphi d \nu \quad \forall \varphi \in C_{0}^{\infty}(\Omega),
$$

where $\langle\cdot, \cdot\rangle$ denotes the duality pairing between $W^{-1, p^{\prime}}(\Omega)$ and $W_{0}^{1, p}(\Omega)$. We shall always identify $f$ and $\nu$. Note that, by the Riesz theorem, for every positive functional $f \in W^{-1, p^{\prime}}(\Omega)$, there exists a Radon measure $\nu$ such that (2.1) holds.

We denote by $\mathcal{M}_{0}^{p}(\Omega)$ the class of all Borel measures which vanish on the sets of $p$-capacity zero and satisfy the following condition:

$$
\mu(B)=\inf \{\mu(A): A \text { p-quasi open, } B \subseteq A \subseteq \Omega\}
$$

for every Borel set $B \subseteq \Omega$. It is well known that every Radon measure which belongs to $W^{-1, p^{\prime}}(\Omega)$ belongs also to $\mathcal{M}_{0}^{p}(\Omega)$ (see [28, section 4.7]).

3. Preliminary results on the relaxed Dirichlet problem with the $p$ Laplace operator. Let $\Omega$ be a bounded open subset of $\mathbf{R}^{N}, N \geq 2$. Let $2 \leq p<+\infty$ and let $\mu \in \mathcal{M}_{0}^{p}(\Omega)$. In the following we shall consider the space $W_{0}^{1, p}(\Omega) \cap L_{\mu}^{p}(\Omega)$ of all functions $u \in W_{0}^{1, p}(\Omega)$ such that $\int_{\Omega}|u|^{p} d \mu<+\infty$. With the norm

$$
\|u\|_{W_{0}^{1, p}(\Omega) \cap L_{\mu}^{p}(\Omega)}=\left(\int_{\Omega}|D u|^{p} d x+\int_{\Omega}|u|^{p} d \mu\right)^{\frac{1}{p}}
$$


the space $W_{0}^{1, p}(\Omega) \cap L_{\mu}^{p}(\Omega)$ is a reflexive Banach space.

Let $f$ be a functional belonging to $\left(W_{0}^{1, p}(\Omega) \cap L_{\mu}^{p}(\Omega)\right)^{\prime}$ (the dual space of $W_{0}^{1, p}(\Omega) \cap$ $\left.L_{\mu}^{p}(\Omega)\right)$ and let us consider the following variational problem:

$$
\left\{\begin{array}{l}
u \in W_{0}^{1, p}(\Omega) \cap L_{\mu}^{p}(\Omega), \\
\int_{\Omega}|D u|^{p-2} D u D v d x+\int_{\Omega}|u|^{p-2} u v d \mu=\langle f, v\rangle \\
\forall v \in W_{0}^{1, p}(\Omega) \cap L_{\mu}^{p}(\Omega) .
\end{array}\right.
$$

Since the operator from $W_{0}^{1, p}(\Omega) \cap L_{\mu}^{p}(\Omega)$ to $\left(W_{0}^{1, p}(\Omega) \cap L_{\mu}^{p}(\Omega)\right)^{\prime}$ mapping $u \in W_{0}^{1, p}(\Omega) \cap$ $L_{\mu}^{p}(\Omega)$ to the functional defined by $\int_{\Omega}|D u|^{p-2} D u D v d x+\int_{\Omega}|u|^{p-2} u v d \mu$ for every $v \in W_{0}^{1, p}(\Omega) \cap L_{\mu}^{p}(\Omega)$ is a maximal monotone operator and the space $W_{0}^{1, p}(\Omega) \cap L_{\mu}^{p}(\Omega)$ is reflexive, we get that there exists a unique solution $u$ of problem (3.1).

Remark 3.1. It is easy to see that the dual of $W_{0}^{1, p}(\Omega) \cap L_{\mu}^{p}(\Omega),\left(W_{0}^{1, p}(\Omega) \cap\right.$ $\left.L_{\mu}^{p}(\Omega)\right)^{\prime}$, coincides with $W^{-1, p^{\prime}}(\Omega)+L_{\mu}^{p^{\prime}}(\Omega)$, so that, in particular, an element of the space $W^{-1, p^{\prime}}(\Omega)$ can be seen as an element of $\left(W_{0}^{1, p}(\Omega) \cap L_{\mu}^{p}(\Omega)\right)^{\prime}$. In what follows, with a slight abuse of notation, $\langle f, v\rangle$ will denote the duality pairing between $\left(W_{0}^{1, p}(\Omega) \cap L_{\mu}^{p}(\Omega)\right)^{\prime}$ and $W_{0}^{1, p}(\Omega) \cap L_{\mu}^{p}(\Omega)$, in the general case $f \in\left(W_{0}^{1, p}(\Omega) \cap L_{\mu}^{p}(\Omega)\right)^{\prime}$, and the duality pairing between $W^{-1, p^{\prime}}(\Omega)$ and $W_{0}^{1, p}(\Omega)$, in the case $f \in W^{-1, p^{\prime}}(\Omega)$.

Many results similar to those given in the linear case (comparison principle, compactness, etc.) have been proved by Dal Maso and Murat (see [16] and [15]) for nonlinear problems of the type (3.1) (in general for nonlinear homogeneous operators).

Proposition 3.2. Let $f_{1}, f_{2} \in W^{-1, p^{\prime}}(\Omega)$ and let $\mu_{1}, \mu_{2} \in \mathcal{M}_{0}^{p}(\Omega)$. Let $u_{1}$, $u_{2} \in W_{0}^{1, p}(\Omega)$ be the solutions of problem (3.1) corresponding to $f_{1}, \mu_{1}$ and to $f_{2}, \mu_{2}$. If $0 \leq f_{1} \leq f_{2}$ and $\mu_{2} \leq \mu_{1}$ in $\Omega$, then $0 \leq u_{1} \leq u_{2}$ p-q.e. in $\Omega$.

Proof. See [15, Proposition 2.7].

In the space $\mathcal{M}_{0}^{p}(\Omega)$ it is possible to introduce a notion of convergence relative to the $p$-Laplace operator, $\Delta_{p} u=\operatorname{div}\left(|D u|^{p-2} D u\right)$.

Definition 3.3. Let $\left(\mu_{n}\right)$ be a sequence of measures of $\mathcal{M}_{0}^{p}(\Omega)$ and let $\mu \in$ $\mathcal{M}_{0}^{p}(\Omega)$. We say that $\left(\mu_{n}\right) \gamma^{-\Delta_{p}}$-converges to the measure $\mu$ if, for every $f \in W^{-1, p^{\prime}}(\Omega)$, the sequence $\left(u_{n}\right)$ of solutions of problems

$$
\left\{\begin{array}{l}
u_{n} \in W_{0}^{1, p}(\Omega) \cap L_{\mu_{n}}^{p}(\Omega), \\
\int_{\Omega}\left|D u_{n}\right|^{p-2} D u_{n} D v d x+\int_{\Omega}\left|u_{n}\right|^{p-2} u_{n} v d \mu_{n}=\langle f, v\rangle \\
\forall v \in W_{0}^{1, p}(\Omega) \cap L_{\mu_{n}}^{p}(\Omega)
\end{array}\right.
$$

converges weakly in $W_{0}^{1, p}(\Omega)$ to the solution u of problem (3.1).

THEOREM 3.4. Every sequence of measures in $\mathcal{M}_{0}^{p}(\Omega)$ contains a $\gamma^{-\Delta_{p}}$-convergent subsequence.

Proof. See [10, Theorem 2.1] or [15, Theorem 6.5].

Many properties of the measure $\mu \in \mathcal{M}_{0}^{p}(\Omega)$ can be studied by means of the 
solution $w$ of the problem

$$
\left\{\begin{array}{l}
w \in W_{0}^{1, p}(\Omega) \cap L_{\mu}^{p}(\Omega), \\
\int_{\Omega}|D w|^{p-2} D w D v d x+\int_{\Omega}|w|^{p-2} w v d \mu=\int_{\Omega} v d x \\
\forall v \in W_{0}^{1, p}(\Omega) \cap L_{\mu}^{p}(\Omega) .
\end{array}\right.
$$

By the comparison principle (Proposition 3.2), the function $w$ is bounded in $L^{\infty}(\Omega)$ by a constant which does not depend on $\mu$ (see [15, section 2]) and satisfies $w \geq 0$ $p$-q.e. in $\Omega$.

TheOREM 3.5. Let $\mu \in \mathcal{M}_{0}^{p}(\Omega)$, let $w$ be the solution of problem (3.3) and let $\nu=1+\Delta_{p} w$ in the sense of $W^{-1, p^{\prime}}(\Omega)$. Then $\nu$ is a nonnegative Radon measure of $W^{-1, p^{\prime}}(\Omega)$ and

$$
\nu(B \cap\{w>0\})=\int_{B} w^{p-1} d \mu
$$

for every Borel set $B \subseteq \Omega$. Reciprocally, we have

$$
\mu(B)= \begin{cases}\int_{B} \frac{1}{w^{p-1}} d \nu & \text { if } C_{p}(B \cap\{w=0\})=0, \\ +\infty & \text { if } C_{p}(B \cap\{w=0\})>0\end{cases}
$$

for any Borel set $B \subseteq \Omega$.

Proof. See [15, Theorem 5.1] and [11, Proposition 3.4] for the linear case.

The next proposition gives two density results which will be useful in what follows.

Proposition 3.6. Let $\mu \in \mathcal{M}_{0}^{p}(\Omega)$ and let $w$ the solution of problem (3.3). Then

(a) the set $\left\{w \psi: \psi \in C_{0}^{\infty}(\Omega)\right\}$ is dense in $W_{0}^{1, p}(\Omega) \cap L_{\mu}^{p}(\Omega)$ and hence in $W_{\mathrm{loc}}^{1, p}(\Omega) \cap L_{\mu}^{p}(\Omega)$.

(b) the set $\Lambda$ of all functions of the form $w \sum_{i=1}^{l} a_{i} 1_{K_{i}}$ where $a_{i} \in \mathbf{R}$ and $K_{i}$ are closed subsets of $\Omega$ such that $w=0 \mu$-a.e. on $K_{i} \cap K_{j}$, with $i \neq j$, is dense in $L_{\mu}^{p}(\Omega)$.

Proof. The proof of part (a) is given in [15, Proposition 5.5]. In order to prove part (b), we consider the measure $\lambda=w^{p} \mu$. Since $w$ belongs to $L_{\mu}^{p}(\Omega)$, the measure $\lambda$ is a Borel bounded measure and therefore the set of all step functions of the form $\sum_{i=1}^{l} a_{i} 1_{K_{i}}$, where $a_{i} \in \mathbf{R}$ and $K_{i}$ are closed subsets of $\Omega$ such that, for $i \neq j$, $\lambda\left(K_{i} \cap K_{j}\right)=0$, is dense in $L_{\lambda}^{p}(\Omega)$. If $u$ belongs to $L_{\mu}^{p}(\Omega)$, then $u / w$ belongs to $L_{\lambda}^{p}(\Omega)$, and for every $\zeta \in \Lambda$ we have

$$
\int_{\Omega}\left|\zeta-\frac{u}{w}\right|^{p} d \lambda=\int_{\Omega}|w \zeta-u|^{p} d \mu
$$

which gives part (b).

Finally the solutions of problems (3.3) are useful to characterize the $\gamma^{-\Delta_{p}}$-convergence in $\mathcal{M}_{0}^{p}(\Omega)$. Let $\left(\mu_{n}\right)$ be a sequence of measures in $\mathcal{M}_{0}^{p}(\Omega)$, and let $w_{n}$ be the solutions of the problems

$$
\left\{\begin{array}{l}
w_{n} \in W_{0}^{1, p}(\Omega) \cap L_{\mu_{n}}^{p}(\Omega), \\
\int_{\Omega}\left|D w_{n}\right|^{p-2} D w_{n} D v d x+\int_{\Omega}\left|w_{n}\right|^{p-2} w_{n} v d \mu_{n}=\int_{\Omega} v d x \\
\forall v \in W_{0}^{1, p}(\Omega) \cap L_{\mu_{n}}^{p}(\Omega) .
\end{array}\right.
$$


The following result characterizes the $\gamma^{-\Delta_{p}}$-convergence in terms of the convergence of the functions $w_{n}$.

THEOREM 3.7. The following conditions are equivalent:

(a) $\left(w_{n}\right)$ converges to $w$ weakly in $W_{0}^{1, p}(\Omega)$;

(b) $\left(\mu_{n}\right) \gamma^{-\Delta_{p}}$-converges to $\mu$.

Proof. See [15, Theorem 6.3] and [11, Theorem 4.3] for the linear case.

Remark 3.8. If $\left(\mu_{n}\right) \gamma^{-\Delta_{p}}$-converges to $\mu$, then the sequence $\left(w_{n}\right)$ converges to $w$ strongly in $W_{0}^{1, r}(\Omega)$ for every $1 \leq r<p$ and hence, a subsequence of $\left(D w_{n}\right)$ converges to $D w$ pointwise a.e. in $\Omega$ (see [15, Theorem 6.8$]$ ).

4. Sequences in the spaces $\boldsymbol{W}^{\mathbf{1}, \boldsymbol{p}} \cap \boldsymbol{L}_{\boldsymbol{\mu}_{n}}^{\boldsymbol{p}}$. In this section $\left(\mu_{n}\right)$ will be a sequence

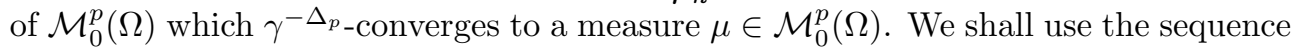
$\left(w_{n}\right)$ of the solutions of problems (3.5) to investigate the behavior of an arbitrary sequence $\left(u_{n}\right)$, with $u_{n} \in W^{1, p}(\Omega) \cap L_{\mu_{n}}^{p}(\Omega)$, which converges weakly in $W^{1, p}(\Omega)$. By Remark 3.8 we may assume that $\left(w_{n}\right)$ and $\left(D w_{n}\right)$, respectively, converge to $w$ and $D w$ pointwise a.e. in $\Omega$.

Let us prove some technical lemmas that will be useful in the remainder of this paper.

Lemma 4.1. Let $\Omega^{\prime}$ be an open subset of $\Omega$. For every $\varphi \in W_{0}^{1, p}\left(\Omega^{\prime}\right) \cap L^{\infty}\left(\Omega^{\prime}\right)$ we have

$$
\lim _{n \rightarrow \infty}\left(\int_{\Omega^{\prime}}\left|D w_{n}\right|^{p} \varphi d x+\int_{\Omega^{\prime}}\left|w_{n}\right|^{p} \varphi d \mu_{n}\right)=\int_{\Omega^{\prime}}|D w|^{p} \varphi d x+\int_{\Omega^{\prime}}|w|^{p} \varphi d \mu .
$$

Proof. Let $\varphi \in W_{0}^{1, p}\left(\Omega^{\prime}\right) \cap L^{\infty}\left(\Omega^{\prime}\right)$ and let us extend $\varphi$ to $\Omega$ by setting $\varphi=0$ in $\Omega \backslash \Omega^{\prime}$. Thus $w_{n} \varphi$ belongs to $W_{0}^{1, p}(\Omega)$, and we can take it as test function in (3.3). Therefore by Remark 3.8 we obtain

$$
\begin{aligned}
\lim _{n \rightarrow \infty} & \left(\int_{\Omega^{\prime}}\left|D w_{n}\right|^{p} \varphi d x+\int_{\Omega^{\prime}}\left|w_{n}\right|^{p} \varphi d \mu_{n}\right) \\
& =\lim _{n \rightarrow \infty}\left(\int_{\Omega^{\prime}} w_{n} \varphi d x-\int_{\Omega^{\prime}}\left|D w_{n}\right|^{p-2} D w_{n} D \varphi w_{n} d x\right) \\
& =\int_{\Omega^{\prime}} w \varphi d x-\int_{\Omega}|D w|^{p-2} D w D \varphi w d x=\int_{\Omega^{\prime}}|D w|^{p} \varphi d x+\int_{\Omega^{\prime}}|w|^{p} \varphi d \mu,
\end{aligned}
$$

which concludes the proof.

Lemma 4.2. Let $\Omega^{\prime}$ be an open subset of $\Omega$. For every $\varphi, \psi \in W^{1, p}\left(\Omega^{\prime}\right) \cap L^{\infty}\left(\Omega^{\prime}\right)$, with $\varphi$ or $\psi$ in $W_{0}^{1, p}\left(\Omega^{\prime}\right)$, we have

$$
\lim _{n \rightarrow \infty}\left(\int_{\Omega^{\prime}}\left|D\left(w_{n} \psi\right)\right|^{p} \varphi d x+\int_{\Omega^{\prime}}\left|w_{n} \psi\right|^{p} \varphi d \mu_{n}\right)=\int_{\Omega^{\prime}}|D(w \psi)|^{p} \varphi d x+\int_{\Omega^{\prime}}|w \psi|^{p} \varphi d \mu .
$$

Proof. Let $\varphi, \psi \in W^{1, p}\left(\Omega^{\prime}\right) \cap L^{\infty}\left(\Omega^{\prime}\right)$, with $\varphi$ or $\psi$ in $W_{0}^{1, p}\left(\Omega^{\prime}\right)$. Since for every $\xi_{1}, \xi_{2} \in \mathbf{R}^{N}$ and for every $p \geq 2$ the following inequality holds

$$
\left.|| \xi_{1}\right|^{p}-\left|\xi_{2}\right|^{p}\left|\leq p\left(\left|\xi_{1}\right|+\left|\xi_{2}\right|\right)^{p-1}\right| \xi_{1}-\xi_{2} \mid
$$

we have

$$
|| \psi D w_{n}+\left.w_{n} D \psi\right|^{p}-\left|\psi D w_{n}\right|^{p}\left|\leq p\left(\left|\psi D w_{n}+w_{n} D \psi\right|+\left|\psi D w_{n}\right|\right)^{p-1}\right| w_{n} D \psi \mid,
$$


where by Remark 3.8 the left-hand side converges pointwise to ||$\psi D w+\left.w D \psi\right|^{p}-$ $|\psi D w|^{p} \mid$ and the right-hand side is uniformly integrable. Then $\left(\left|D\left(w_{n} \psi\right)\right|^{p}-\left|\psi D w_{n}\right|^{p}\right)$ converges to $|D(w \psi)|^{p}-|\psi D w|^{p}$ strongly in $L^{1}\left(\Omega^{\prime}\right)$. This implies that

$\int_{\Omega^{\prime}}\left|D\left(w_{n} \psi\right)\right|^{p} \varphi d x=\int_{\Omega^{\prime}}|D(w \psi)|^{p} \varphi d x-\int_{\Omega^{\prime}}|D w|^{p}|\psi|^{p} \varphi d x+\int_{\Omega^{\prime}}\left|D w_{n}\right|^{p}|\psi|^{p} \varphi d x+o_{n}$

and therefore the conclusion follows from Lemma 4.1.

Lemma 4.3. Let $\Omega^{\prime}$ be an open subset of $\Omega$, let $u \in W^{1, p}\left(\Omega^{\prime}\right) \cap L_{\mu}^{p}\left(\Omega^{\prime}\right)$, and let $\left(\psi_{m}\right)$ be a sequence of functions in $C_{0}^{\infty}\left(\Omega^{\prime}\right)$ such that $\left(w \psi_{m}\right)$ converges to u strongly in $W_{\mathrm{loc}}^{1, p}\left(\Omega^{\prime}\right) \cap L_{\mu}^{p}\left(\Omega^{\prime}\right)$. Then

$$
\lim _{m \rightarrow \infty} \lim _{n \rightarrow \infty}\left(\int_{\Omega^{\prime}}\left|D\left(w_{n} \psi_{m}-u\right)\right|^{p} \varphi d x+\int_{\Omega^{\prime}}\left|w_{n} \psi_{m}\right|^{p} \varphi d \mu_{n}\right)=\int_{\Omega^{\prime}}|u|^{p} \varphi d \mu
$$

for every $\varphi \in W_{c}^{1, p}\left(\Omega^{\prime}\right) \cap L^{\infty}\left(\Omega^{\prime}\right)$.

Proof. As in the proof of Lemma 4.2, $\left(\left|D\left(w_{n} \psi_{m}-u\right)\right|^{p}-\left|D\left(w_{n} \psi_{m}\right)\right|^{p}\right)$ converges to $\left|D\left(w \psi_{m}-u\right)\right|^{p}-\left|D\left(w \psi_{m}\right)\right|^{p}$ strongly in $L^{1}\left(\Omega^{\prime}\right)$ as $n \rightarrow \infty$. Let $\varphi \in W_{c}^{1, p}\left(\Omega^{\prime}\right) \cap L^{\infty}\left(\Omega^{\prime}\right)$; thus, by Lemma 4.2, we get

$$
\begin{gathered}
\int_{\Omega^{\prime}}\left|D\left(w_{n} \psi_{m}-u\right)\right|^{p} \varphi d x+\int_{\Omega^{\prime}}\left|w_{n} \psi_{m}\right|^{p} \varphi d \mu_{n}=\int_{\Omega^{\prime}}\left(\left|D\left(w_{n} \psi_{m}-u\right)\right|^{p}-\left|D\left(w_{n} \psi_{m}\right)\right|^{p}\right) \varphi d x \\
+\int_{\Omega^{\prime}}\left|D\left(w_{n} \psi_{m}\right)\right|^{p} \varphi d x+\int_{\Omega^{\prime}}\left|w_{n} \psi_{m}\right|^{p} \varphi d \mu_{n}=\int_{\Omega^{\prime}}\left(\left|D\left(w \psi_{m}-u\right)\right|^{p}-\left|D\left(w \psi_{m}\right)\right|^{p}\right) \varphi d x \\
\quad+\int_{\Omega^{\prime}}\left|D\left(w \psi_{m}\right)\right|^{p} \varphi d x+\int_{\Omega^{\prime}}\left|w \psi_{m}\right|^{p} \varphi d \mu+o_{n}=\int_{\Omega^{\prime}}|u|^{p} \varphi d \mu+o_{m, n} .
\end{gathered}
$$

The conclusion follows by taking the limit first as $n \rightarrow \infty$ and then as $m \rightarrow \infty$.

Let $\Omega^{\prime}$ be an open subset of $\Omega$. The following theorem shows that if a sequence $\left(u_{n}\right)$, with $u_{n} \in W^{1, p}\left(\Omega^{\prime}\right) \cap L_{\mu_{n}}^{p}\left(\Omega^{\prime}\right)$, converges weakly in $W^{1, p}\left(\Omega^{\prime}\right)$ to a function $u \in W^{1, p}\left(\Omega^{\prime}\right)$, and there exists a constant $C>0$ such that

$$
\int_{\Omega^{\prime}}\left|u_{n}\right|^{p} d \mu_{n} \leq C
$$

for every $n \in \mathbf{N}$, then the function $u$ belongs to $L_{\mu}^{p}\left(\Omega^{\prime}\right)$.

THEOREM 4.4. Let $\left(u_{n}\right)$ be a sequence such that $u_{n} \in W^{1, p}\left(\Omega^{\prime}\right) \cap L_{\mu_{n}}^{p}\left(\Omega^{\prime}\right)$. Suppose that $\left(u_{n}\right)$ converges weakly in $W^{1, p}\left(\Omega^{\prime}\right)$ to some function $u$. Then

$$
\liminf _{n \rightarrow \infty}\left(\int_{\Omega^{\prime}}\left|D u_{n}\right|^{p} d x+\int_{\Omega^{\prime}}\left|u_{n}\right|^{p} d \mu_{n}\right) \geq \int_{\Omega^{\prime}}|D u|^{p} d x+\int_{\Omega^{\prime}}|u|^{p} d \mu .
$$

In particular, if (4.5) holds, then $u \in W^{1, p}\left(\Omega^{\prime}\right) \cap L_{\mu}^{p}\left(\Omega^{\prime}\right)$.

The result of Theorem 4.4 can be obtained as a direct consequence of the $\Gamma$ convergence of the functionals $\int_{\Omega^{\prime}}\left|D u_{n}\right|^{p} d x+\int_{\Omega^{\prime}}\left|u_{n}\right|^{p} d \mu_{n}$ to the functional $\int_{\Omega^{\prime}}|D u|^{p} d x+$ $\int_{\Omega^{\prime}}|u|^{p} d \mu$ proved in [10]. For the sake of completeness we shall give an alternative proof of Theorem 4.4 which does not involve $\Gamma$-convergence theory. Before proving Theorem 4.4, let us prove two preliminary lemmas.

LEMma 4.5. Let $\left(u_{n}\right)$ be a sequence such that $u_{n} \in W^{1, p}\left(\Omega^{\prime}\right) \cap L_{\mu_{n}}^{p}\left(\Omega^{\prime}\right)$ and such that (4.5) holds. Suppose that $\left(u_{n}\right)$ converges weakly in $W^{1, p}\left(\Omega^{\prime}\right)$ to some function $u$. Then $\{u=0\}_{\Omega^{\prime}} \supseteq\{w=0\}_{\Omega^{\prime}}$. 
Proof. Taking into account the decomposition $u_{n}=u_{n}^{+}-u_{n}^{-}$, where $u_{n}^{+}$and $u_{n}^{-}$ denote respectively the positive and the negative part of $u_{n}$, it is not restrictive to assume $u_{n} \geq 0$ p-q.e. in $\Omega^{\prime}$.

We shall prove first the result in the special case where the functions $u_{n}$ and $u$ belong to $W_{0}^{1, p}\left(\Omega^{\prime}\right)$, and we shall suppose, also, that there exists a constant $K>0$ such that $u_{n} \leq K p$-q.e. in $\Omega^{\prime}$ and hence $u \leq K$ p-q.e. in $\Omega^{\prime}$.

For every $m \in \mathbf{N}$ let us consider the sequence $\left(u_{n}^{m}\right)$ of the solutions of the problems

$$
\left\{\begin{array}{l}
u_{n}^{m} \in W_{0}^{1, p}\left(\Omega^{\prime}\right) \cap L_{\mu_{n}}^{p}\left(\Omega^{\prime}\right), \\
\int_{\Omega^{\prime}}\left|D u_{n}^{m}\right|^{p-2} D u_{n}^{m} D v d x+\int_{\Omega^{\prime}}\left|u_{n}^{m}\right|^{p-2} u_{n}^{m} v d \mu_{n} \\
\quad=m \int_{\Omega^{\prime}}\left(\left|u_{n}\right|^{p-2} u_{n}-\left|u_{n}^{m}\right|^{p-2} u_{n}^{m}\right) v d x \\
\forall v \in W_{0}^{1, p}\left(\Omega^{\prime}\right) \cap L_{\mu_{n}}^{p}\left(\Omega^{\prime}\right),
\end{array}\right.
$$

which, extended to $\Omega$ by setting $u_{n}^{m}=0$ in $\Omega \backslash \Omega^{\prime}$, are also the solutions of the following equivalent problems:

$$
\left\{\begin{array}{l}
u_{n}^{m} \in W_{0}^{1, p}(\Omega) \cap L_{\hat{\mu}_{n}}^{p}(\Omega), \\
\int_{\Omega}\left|D u_{n}^{m}\right|^{p-2} D u_{n}^{m} D v d x+\int_{\Omega}\left|u_{n}^{m}\right|^{p-2} u_{n}^{m} v d \hat{\mu}_{n} \\
\quad=m \int_{\Omega}\left(\left|u_{n}\right|^{p-2} u_{n}-\left|u_{n}^{m}\right|^{p-2} u_{n}^{m}\right) v d x \\
\forall v \in W_{0}^{1, p}(\Omega) \cap L_{\hat{\mu}_{n}}^{p}(\Omega),
\end{array}\right.
$$

where $\hat{\mu}_{n}$ is the measure defined by

$$
\hat{\mu}_{n}(B)= \begin{cases}\mu_{n}(B) & \text { if } C_{p}\left(B \cap\left(\Omega \backslash \Omega^{\prime}\right)\right)=0, \\ +\infty & \text { if } C_{p}\left(B \cap\left(\Omega \backslash \Omega^{\prime}\right)\right)>0\end{cases}
$$

for any Borel set $B \subseteq \Omega$. By the comparison principle (Proposition 3.2) we have that

$$
0 \leq u_{n}^{m} \leq m^{\frac{1}{p-1}} K w_{n} \quad p \text {-q.e. in } \Omega .
$$

By taking in (4.7) $u_{n}^{m}-u_{n}$ as a test function we get

$$
\begin{gathered}
\int_{\Omega^{\prime}}\left(\left|D u_{n}^{m}\right|^{p-2} D u_{n}^{m}-\left|D u_{n}\right|^{p-2} D u_{n}\right) D\left(u_{n}^{m}-u_{n}\right) d x \\
+\int_{\Omega^{\prime}}\left(\left|u_{n}^{m}\right|^{p-2} u_{n}^{m}-\left|u_{n}\right|^{p-2} u_{n}\right)\left(u_{n}^{m}-u_{n}\right) d \mu_{n} \\
+m \int_{\Omega^{\prime}}\left(\left|u_{n}^{m}\right|^{p-2} u_{n}^{m}-\left|u_{n}\right|^{p-2} u_{n}\right)\left(u_{n}^{m}-u_{n}\right) d x \\
=-\int_{\Omega^{\prime}}\left|D u_{n}\right|^{p-2} D u_{n} D\left(u_{n}^{m}-u_{n}\right) d x-\int_{\Omega^{\prime}}\left|u_{n}\right|^{p-2} u_{n}\left(u_{n}^{m}-u_{n}\right) d \mu_{n} .
\end{gathered}
$$

Since for every $\xi_{1}, \xi_{2} \in \mathbf{R}^{N}$ and for every $p \geq 2$ we have

$$
\left(\left|\xi_{1}\right|^{p-2} \xi_{1}-\left|\xi_{2}\right|^{p-2} \xi_{2}\right)\left(\xi_{1}-\xi_{2}\right) \geq 2^{2-p}\left|\xi_{1}-\xi_{2}\right|^{p},
$$


applying Young's inequality in (4.10) we get

$$
\begin{gathered}
2^{2-p} \int_{\Omega^{\prime}}\left|D\left(u_{n}^{m}-u_{n}\right)\right|^{p} d x+2^{2-p} \int_{\Omega^{\prime}}\left|u_{n}^{m}-u_{n}\right|^{p} d \mu_{n}+2^{2-p} m \int_{\Omega^{\prime}}\left|u_{n}^{m}-u_{n}\right|^{p} d x \\
\leq \frac{1}{\varepsilon^{p^{\prime}} p^{\prime}}\left(\int_{\Omega^{\prime}}\left|D u_{n}\right|^{p} d x+\int_{\Omega^{\prime}}\left|u_{n}\right|^{p} d \mu_{n}\right) \\
+\frac{\varepsilon^{p}}{p}\left(\int_{\Omega^{\prime}}\left|D\left(u_{n}^{m}-u_{n}\right)\right|^{p} d x+\int_{\Omega^{\prime}}\left|u_{n}^{m}-u_{n}\right|^{p} d \mu_{n}\right)
\end{gathered}
$$

where $\varepsilon>0$ is an arbitrary real number. Since $\left(u_{n}\right)$ is bounded in $W_{0}^{1, p}\left(\Omega^{\prime}\right)$ and $(4.5)$ holds, by choosing $\varepsilon$ small enough we obtain that there exists a constant $C>0$ such that

$$
\int_{\Omega^{\prime}}\left|D\left(u_{n}^{m}-u_{n}\right)\right|^{p} d x+m \int_{\Omega^{\prime}}\left|u_{n}^{m}-u_{n}\right|^{p} d x \leq C .
$$

By (4.12) we have that the sequence $\left(u_{n}^{m}\right)$ is bounded in $W_{0}^{1, p}\left(\Omega^{\prime}\right)$, uniformly in $m$ and $n$. Then for every $m \in \mathbf{N}$ there exists a subsequence of $\left(u_{n}^{m}\right)$ (we can choose the subsequence independent of $m$ ) which converges to a function $u^{m}$ weakly in $W_{0}^{1, p}\left(\Omega^{\prime}\right)$. By the weak lower semicontinuity of the norm, the sequence $\left(u^{m}\right)$ is also bounded in $W_{0}^{1, p}\left(\Omega^{\prime}\right)$. Moreover by (4.12) we get

$$
\int_{\Omega^{\prime}}\left|u^{m}-u\right|^{p} d x=\lim _{n \rightarrow \infty} \int_{\Omega^{\prime}}\left|u_{n}^{m}-u_{n}\right|^{p} d x \leq \frac{C}{m},
$$

and hence $\left(u^{m}\right)$ converges weakly to $u$ in $W_{0}^{1, p}\left(\Omega^{\prime}\right)$. By (4.9) we have that $\left|u^{m}\right| \leq$ $m^{1 /(p-1)} K w$ p-q.e. in $\Omega^{\prime}$ and hence $u^{m}$ belongs to the set $\mathcal{K}=\left\{v \in W_{0}^{1, p}\left(\Omega^{\prime}\right): v=\right.$ $0 p$-q.e. in $\left.\{w=0\}_{\Omega^{\prime}}\right\}$. Since $\mathcal{K}$ is convex and closed in $W_{0}^{1, p}\left(\Omega^{\prime}\right)$, it is weakly closed. Therefore $u \in \mathcal{K}$ and hence $\{u=0\}_{\Omega^{\prime}} \supseteq\{w=0\}_{\Omega^{\prime}}$.

Finally let us consider the general case where the sequence $\left(u_{n}\right)$ is not bounded in $L^{\infty}\left(\Omega^{\prime}\right)$ but $u_{n} \in W^{1, p}\left(\Omega^{\prime}\right) \cap L_{\mu_{n}}^{p}\left(\Omega^{\prime}\right)$, satisfies (4.5), and converges weakly in $W^{1, p}\left(\Omega^{\prime}\right)$ to $u$. Let $\Phi$ be a function in $W_{0}^{1, \infty}\left(\Omega^{\prime}\right)$, with $\Phi>0$ in $\Omega^{\prime}$, and for every $n \in \mathbf{N}$ let $T_{1} u_{n}$ be the truncation at the level 1 of $u_{n}$. Since $\Phi T_{1} u_{n} \in W_{0}^{1, p}\left(\Omega^{\prime}\right) \cap L_{\mu_{n}}^{p}\left(\Omega^{\prime}\right)$ and the sequence $\left(\Phi T_{1} u_{n}\right)$ satisfies $(4.5)$, is bounded in $L^{\infty}\left(\Omega^{\prime}\right)$, and converges weakly in $W_{0}^{1, p}\left(\Omega^{\prime}\right)$ to $\Phi T_{1} u$, by the previous step we can conclude that $\left\{\Phi T_{1} u=0\right\}_{\Omega^{\prime}} \supseteq\{w=$ $0\}_{\Omega^{\prime}}$ and hence $\{u=0\}_{\Omega^{\prime}} \supseteq\{w=0\}_{\Omega^{\prime}}$.

Lemma 4.6. Let $\left(v_{n}\right)$, with $v_{n} \in W^{1, p}\left(\Omega^{\prime}\right) \cap L_{\mu_{n}}^{p}\left(\Omega^{\prime}\right)$, be a sequence which converges to a function $v$ weakly in $W^{1, p}\left(\Omega^{\prime}\right)$, and suppose that there exists a constant $C>0$ such that

$$
\int_{\Omega^{\prime}}\left|v_{n}\right|^{p} d \mu_{n} \leq C
$$

for every $n \in \mathbf{N}$. Then we have

$$
\begin{gathered}
\lim _{n \rightarrow \infty}\left(\int_{\Omega^{\prime}} \varphi\left|D\left(w_{n} \psi\right)\right|^{p-2} D\left(w_{n} \psi\right) D v_{n} d x+\int_{\Omega^{\prime}} \varphi\left|w_{n} \psi\right|^{p-2} w_{n} \psi v_{n} d \mu_{n}\right) \\
=\int_{\Omega^{\prime}} \varphi|D(w \psi)|^{p-2} D(w \psi) D v d x+\int_{\Omega^{\prime}} \varphi|w \psi|^{p-2} w \psi v d \mu
\end{gathered}
$$

for every $\psi \in W^{1, p}\left(\Omega^{\prime}\right) \cap L^{\infty}\left(\Omega^{\prime}\right)$ and for every $\varphi \in W_{0}^{1, p}\left(\Omega^{\prime}\right) \cap L^{\infty}\left(\Omega^{\prime}\right)$. 
Proof. Let $\psi \in W^{1, p}\left(\Omega^{\prime}\right) \cap L^{\infty}\left(\Omega^{\prime}\right)$ and $\varphi \in W_{0}^{1, p}\left(\Omega^{\prime}\right) \cap L^{\infty}\left(\Omega^{\prime}\right)$. Since for every $p \geq 2$ the following inequality holds

$$
\left.|| \xi_{1}\right|^{p-2} \xi_{1}-\left|\xi_{2}\right|^{p-2} \xi_{2}\left|\leq(p-1)\left(\left|\xi_{1}\right|+\left|\xi_{2}\right|\right)^{p-2}\right| \xi_{1}-\xi_{2} \mid
$$

for every $\xi_{1}, \xi_{2} \in \mathbf{R}^{N}$, as in Lemma 4.2 , we can conclude that $\left(\left|D\left(w_{n} \psi\right)\right|^{p-2} D\left(w_{n} \psi\right)-\right.$ $\left.\left|\psi D w_{n}\right|^{p-2} \psi D w_{n}\right)$ converges to $|D(w \psi)|^{p-2} D(w \psi)-|\psi D w|^{p-2} \psi D w$ strongly in $L^{p^{\prime}}\left(\Omega^{\prime}, \mathbf{R}^{N}\right)$. Thus

$$
\begin{gathered}
\lim _{n \rightarrow \infty}\left(\int_{\Omega^{\prime}} \varphi\left|D\left(w_{n} \psi\right)\right|^{p-2} D\left(w_{n} \psi\right) D v_{n} d x-\int_{\Omega^{\prime}} \varphi\left|\psi D w_{n}\right|^{p-2} \psi D w_{n} D v_{n} d x\right) \\
=\int_{\Omega^{\prime}}\left(|D(w \psi)|^{p-2} D(w \psi)-|\psi D w|^{p-2} \psi D w\right) D v \varphi d x .
\end{gathered}
$$

In order to conclude the proof it is enough to show that

$$
\begin{gathered}
\lim _{n \rightarrow \infty}\left(\int_{\Omega^{\prime}} \varphi\left|\psi D w_{n}\right|^{p-2} \psi D w_{n} D v_{n} d x+\int_{\Omega^{\prime}} \varphi\left|w_{n} \psi\right|^{p-2} w_{n} \psi v_{n} d \mu_{n}\right) \\
=\int_{\Omega^{\prime}} \varphi|\psi D w|^{p-2} \psi D w D v d x+\int_{\Omega^{\prime}} \varphi|\psi|^{p-2} \psi v d \nu
\end{gathered}
$$

where $\nu \in W^{-1, p^{\prime}}(\Omega)$ is the Radon measure defined in Theorem 3.5. Indeed, since by Lemma 4.5 we have that $\{v=0\}_{\Omega^{\prime}} \supseteq\{w=0\}_{\Omega^{\prime}}$, by (3.4) we get

$$
\int_{\Omega^{\prime}} \varphi|\psi|^{p-2} \psi v d \nu=\int_{\{w>0\}_{\Omega^{\prime}}} \varphi|\psi|^{p-2} \psi v d \nu=\int_{\Omega^{\prime}} \varphi w^{p-1}|\psi|^{p-2} \psi v d \mu
$$

therefore the conclusion follows from (4.16) and (4.17).

It remains to prove (4.17). Let us consider $\phi \in W_{0}^{1, \infty}\left(\Omega^{\prime}\right)$. Taking $\phi v_{n} \in$ $W_{0}^{1, p}\left(\Omega^{\prime}\right) \cap L_{\mu_{n}}^{p}\left(\Omega^{\prime}\right) \subset W_{0}^{1, p}(\Omega) \cap L_{\mu_{n}}^{p}(\Omega)$ as a test function in problem (3.5), and taking into account that $\nu=1+\Delta_{p} w$ in $W^{-1, p^{\prime}}(\Omega)$ (Theorem 3.5), we obtain

$$
\begin{gathered}
\lim _{n \rightarrow \infty} \int_{\Omega^{\prime}} \phi\left|D w_{n}\right|^{p-2} D w_{n} D v_{n} d x+\int_{\Omega^{\prime}} \phi\left|w_{n}\right|^{p-2} w_{n} v_{n} d \mu_{n} \\
=\lim _{n \rightarrow \infty} \int_{\Omega^{\prime}} \phi v_{n} d x-\int_{\Omega^{\prime}}\left|D w_{n}\right|^{p-2} D w_{n} D \phi v_{n} d x \\
=\int_{\Omega^{\prime}} \phi v d x-\int_{\Omega^{\prime}}|D w|^{p-2} D w D \phi v d x=\int_{\Omega^{\prime}} \phi|D w|^{p-2} D w D v d x+\int_{\Omega^{\prime}} \phi v d \nu .
\end{gathered}
$$

We have to prove that (4.18) holds for every $\phi \in W_{0}^{1, p}\left(\Omega^{\prime}\right) \cap L^{\infty}\left(\Omega^{\prime}\right)$. Let $\phi \in$ $W_{0}^{1, p}\left(\Omega^{\prime}\right) \cap L^{\infty}\left(\Omega^{\prime}\right)$. Since $\nu$ is a Radon measure in $W^{-1, p^{\prime}}(\Omega)$, it is possible to construct a sequence $\left(\phi_{m}\right)$ of functions in $W_{0}^{1, \infty}\left(\Omega^{\prime}\right)$ bounded in $L^{\infty}\left(\Omega^{\prime}\right)$, which converges to $\phi$ a.e. and $\nu$-a.e. in $\Omega^{\prime}$. By (4.18) we have

$$
\begin{gathered}
\left.\left|\int_{\Omega^{\prime}} \phi\right| D w_{n}\right|^{p-2} D w_{n} D v_{n} d x+\int_{\Omega^{\prime}} \phi\left|w_{n}\right|^{p-2} w_{n} v_{n} d \mu_{n}-\int_{\Omega^{\prime}} \phi|D w|^{p-2} D w D v d x-\int_{\Omega^{\prime}} \phi v d \nu \mid \\
\leq\left.\left|\int_{\Omega^{\prime}}\left(\phi-\phi_{m}\right)\right| D w_{n}\right|^{p-2} D w_{n} D v_{n} d x+\int_{\Omega^{\prime}}\left(\phi-\phi_{m}\right)\left|w_{n}\right|^{p-2} w_{n} v_{n} d \mu_{n} \mid \\
\quad+\left.\left|\int_{\Omega^{\prime}}\left(\phi_{m}-\phi\right)\right| D w\right|^{p-2} D w D v d x+\int_{\Omega^{\prime}}\left(\phi_{m}-\phi\right) v d \nu \mid+o_{n} .
\end{gathered}
$$


By the dominated convergence theorem the limit as $m \rightarrow \infty$ of the second term in the right-hand side of (4.19) is zero. It remains to estimate the first term of the right-hand side of (4.19). Since $\left(\phi_{m}\right)$ is bounded in $L^{\infty}\left(\Omega^{\prime}\right)$, by Hölder's inequality, (4.13), and Lemma 4.1 we obtain

$$
\begin{gathered}
\left.\left|\int_{\Omega^{\prime}}\left(\phi-\phi_{m}\right)\right| D w_{n}\right|^{p-2} D w_{n} D v_{n} d x+\int_{\Omega^{\prime}}\left(\phi-\phi_{m}\right)\left|w_{n}\right|^{p-2} w_{n} v_{n} d \mu_{n} \mid \\
\leq C\left(\int_{\Omega^{\prime}}\left|D w_{n}\right|^{p}\left|\phi-\phi_{m}\right|^{\frac{p}{(p-1)}} d x+\int_{\Omega^{\prime}}\left|w_{n}\right|^{p}\left|\phi-\phi_{m}\right|^{\frac{p}{(p-1)}} d \mu_{n}\right)^{\frac{p-1}{p}} \\
=C\left(\int_{\Omega^{\prime}}|D w|^{p}\left|\phi-\phi_{m}\right|^{\frac{p}{(p-1)}} d x+\int_{\Omega^{\prime}}|w|^{p}\left|\phi-\phi_{m}\right|^{\frac{p}{(p-1)}} d \mu\right)^{\frac{p-1}{p}}+o_{n}=o_{m, n},
\end{gathered}
$$

where $C$ is a positive constant independent of $n$ and $m$ and where for the last limit we used the dominated convergence theorem. Finally (4.17) follows immediately from (4.18) by choosing $\phi=\varphi|\psi|^{p-2} \psi$.

We are now in a position to prove Theorem 4.4.

Proof of Theorem 4.4. If $\liminf _{n \rightarrow \infty} \int_{\Omega^{\prime}}\left|u_{n}\right|^{p} d \mu_{n}=+\infty$, then inequality (4.6) is trivially satisfied; otherwise it is not restrictive to suppose that (4.5) holds. Let $\psi \in W^{1, p}\left(\Omega^{\prime}\right) \cap L^{\infty}\left(\Omega^{\prime}\right)$, and let $\varphi \in W_{0}^{1, p}\left(\Omega^{\prime}\right) \cap L^{\infty}\left(\Omega^{\prime}\right)$ with $\varphi \geq 0$. Since for every $\xi_{1}, \xi_{2} \in \mathbf{R}^{N}$, by the convexity of the function $|\cdot|^{p}$, the following inequality holds:

$$
\left|\xi_{1}\right|^{p}-\left|\xi_{2}\right|^{p} \geq p\left|\xi_{2}\right|^{p-2} \xi_{2}\left(\xi_{1}-\xi_{2}\right)
$$

we have

$$
\begin{gathered}
\int_{\Omega^{\prime}} \varphi\left|D u_{n}\right|^{p} d x+\int_{\Omega^{\prime}} \varphi\left|u_{n}\right|^{p} d \mu_{n} \geq \int_{\Omega^{\prime}} \varphi\left|D\left(w_{n} \psi\right)\right|^{p} d x+\int_{\Omega^{\prime}} \varphi\left|w_{n} \psi\right|^{p} d \mu_{n} \\
+p \int_{\Omega^{\prime}}\left|D\left(w_{n} \psi\right)\right|^{p-2} D\left(w_{n} \psi\right) D\left(u_{n}-w_{n} \psi\right) \varphi d x+p \int_{\Omega^{\prime}}\left|w_{n} \psi\right|^{p-2} w_{n} \psi\left(u_{n}-w_{n} \psi\right) \varphi d \mu_{n} .
\end{gathered}
$$

By Lemmas 4.2 and 4.6 we get

$$
\begin{gathered}
\liminf _{n \rightarrow \infty}\left(\int_{\Omega^{\prime}} \varphi\left|D u_{n}\right|^{p} d x+\int_{\Omega^{\prime}} \varphi\left|u_{n}\right|^{p} d \mu_{n}\right) \\
\geq \int_{\Omega^{\prime}} \varphi|D(w \psi)|^{p} d x+\int_{\Omega^{\prime}} \varphi|w \psi|^{p} d \mu \\
+p \int_{\Omega^{\prime}}|D(w \psi)|^{p-2} D(w \psi) D(u-w \psi) \varphi d x+p \int_{\Omega^{\prime}}|w \psi|^{p-2} w \psi(u-w \psi) \varphi d \mu .
\end{gathered}
$$

Assume that $u \in L^{\infty}\left(\Omega^{\prime}\right)$. Let $\varepsilon>0$ and let us choose in (4.21) $\psi=\frac{u}{w \vee \varepsilon}$ and $\varphi=\phi R_{\varepsilon}(w)$, with $0 \leq \phi \leq 1, \phi \in W_{0}^{1, p}\left(\Omega^{\prime}\right) \cap L^{\infty}\left(\Omega^{\prime}\right)$, and $R_{\varepsilon}: \mathbf{R} \mapsto \mathbf{R}$ defined by

$$
R_{\varepsilon}(s)= \begin{cases}0 & \text { if } s \leq \varepsilon \\ \frac{s}{\varepsilon}-1 & \text { if } \varepsilon \leq s \leq 2 \varepsilon \\ 1 & \text { if } 2 \varepsilon \leq s<+\infty\end{cases}
$$

Since $w \psi=u$ p-q.e. in $\{w>\varepsilon\}$ and $\phi R_{\varepsilon}(w)=0$ p-q.e. in $\{w \leq \varepsilon\}$, by (4.21) we have

$$
\begin{gathered}
\liminf _{n \rightarrow \infty}\left(\int_{\Omega^{\prime}}\left|D u_{n}\right|^{p} d x+\int_{\Omega^{\prime}}\left|u_{n}\right|^{p} d \mu_{n}\right) \\
\geq \int_{\Omega^{\prime} \cap\{w>\varepsilon\}} R_{\varepsilon}(w) \phi|D u|^{p} d x+\int_{\Omega^{\prime} \cap\{w>\varepsilon\}} R_{\varepsilon}(w) \phi|u|^{p} d \mu,
\end{gathered}
$$


which, by the monotone convergence theorem, implies

$$
\begin{aligned}
& \liminf _{n \rightarrow \infty}\left(\int_{\Omega^{\prime}}\left|D u_{n}\right|^{p} d x+\int_{\Omega^{\prime}}\left|u_{n}\right|^{p} d \mu_{n}\right) \\
& \geq \int_{\Omega^{\prime} \cap\{w>0\}} \phi|D u|^{p} d x+\int_{\Omega^{\prime} \cap\{w>0\}} \phi|u|^{p} d \mu
\end{aligned}
$$

for every $\phi \in W_{0}^{1, p}\left(\Omega^{\prime}\right) \cap L^{\infty}\left(\Omega^{\prime}\right)$ with $0 \leq \phi \leq 1$. Since $D u=0$ a.e. in $\{u=0\}_{\Omega^{\prime}}$ and by Lemma $4.5\{u=0\}_{\Omega^{\prime}} \supseteq\{w=0\}_{\Omega^{\prime}}$, estimate (4.23) may be written as

$$
\liminf _{n \rightarrow \infty}\left(\int_{\Omega^{\prime}}\left|D u_{n}\right|^{p} d x+\int_{\Omega^{\prime}}\left|u_{n}\right|^{p} d \mu_{n}\right) \geq \int_{\Omega^{\prime}} \phi|D u|^{p} d x+\int_{\Omega^{\prime}} \phi|u|^{p} d \mu .
$$

Thus, by the monotone convergence theorem, we deduce that $u \in L_{\mu}^{p}\left(\Omega^{\prime}\right)$ and (4.6) holds. If $u$ does not belong to $L^{\infty}\left(\Omega^{\prime}\right)$, it is enough to apply the previous step to the sequence of truncations $T_{k}\left(u_{n}\right)$ with $k \in \mathbf{N}$. Then we have

$$
\begin{gathered}
\liminf _{n \rightarrow \infty}\left(\int_{\Omega^{\prime}}\left|D u_{n}\right|^{p} d x+\int_{\Omega^{\prime}}\left|u_{n}\right|^{p} d \mu_{n}\right) \geq \liminf _{n \rightarrow \infty}\left(\int_{\Omega^{\prime}}\left|D T_{k}\left(u_{n}\right)\right|^{p} d x+\int_{\Omega^{\prime}}\left|T_{k}\left(u_{n}\right)\right|^{p} d \mu_{n}\right) \\
\geq \int_{\Omega^{\prime}}\left|D T_{k}(u)\right|^{p} d x+\int_{\Omega^{\prime}}\left|T_{k}(u)\right|^{p} d \mu .
\end{gathered}
$$

We conclude the proof by the monotone convergence theorem, taking the limit as $k \rightarrow \infty$.

5. Relaxed Dirichlet problems with monotone operators. Let $A$ be the monotone operator defined from $W^{1, p}\left(\Omega, \mathbf{R}^{M}\right)$ to $W^{-1, p^{\prime}}\left(\Omega, \mathbf{R}^{M}\right)$, with $2 \leq p<+\infty$ and $M \geq 2$, mapping $u \in W^{1, p}\left(\Omega, \mathbf{R}^{M}\right)$ in $A u=-\operatorname{div}(a(x, D u)) \in W^{-1, p^{\prime}}\left(\Omega, \mathbf{R}^{M}\right)$, where $a: \Omega \times \mathbf{M}^{M \times N} \mapsto \mathbf{M}^{M \times N}$ is a Carathéodory function. We shall assume that the function $a$ satisfies the following conditions:

(i) there exists a constant $\alpha>0$ such that

$$
\left(a\left(x, \xi_{1}\right)-a\left(x, \xi_{2}\right)\right)\left(\xi_{1}-\xi_{2}\right) \geq \alpha\left|\xi_{1}-\xi_{2}\right|^{p}
$$

for every $\xi_{1}, \xi_{2} \in \mathbf{M}^{M \times N}$ and for a.e. $x \in \Omega$;

(ii) there exists a constant $\beta>0$ and a function $h \in L^{\frac{p}{p-2}}(\Omega)$ such that

$$
\left|a\left(x, \xi_{1}\right)-a\left(x, \xi_{2}\right)\right| \leq \beta\left(h(x)+\left(\left|\xi_{1}\right|+\left|\xi_{2}\right|\right)^{p-2}\right)\left|\xi_{1}-\xi_{2}\right|
$$

for every $\xi_{1}, \xi_{2} \in \mathbf{M}^{M \times N}$ and for a.e. $x \in \Omega$;

(iii) $a(x, 0)=0$ a.e. in $\Omega$.

These hypotheses imply in particular that the following conditions hold:

(iv) there exists a constant $\eta>0$ and a function $k \in L^{p^{\prime}}(\Omega)$ such that

$$
|a(x, \xi)| \leq k(x)+\eta|\xi|^{p-1}
$$

for every $\xi \in \mathbf{M}^{M \times N}$ and for a.e. $x \in \Omega$;

(v) $a(x, \xi) \xi \geq \alpha|\xi|^{p}$ for every $\xi \in \mathbf{M}^{M \times N}$ and a.e. $x \in \Omega$.

We shall see in section 10 that the results proved in what follows hold for a class of operators which satisfy more general conditions than (i)-(iv) above.

Given three positive constants $c_{1}, c_{2}$, and $\sigma$, with $0<\sigma \leq 1$, let us define the class $\mathcal{F}\left(c_{1}, c_{2}, \sigma\right)$ of all vector functions $F: \Omega \times \mathbf{R}^{M} \mapsto \mathbf{R}^{M}$ such that the following properties are satisfied: 
(I) $F(x, s)$ is a Borel function;

(II) for every $s_{1}, s_{2} \in \mathbf{R}^{M}$ and for every $x \in \Omega$ we have

$$
\left(F\left(x, s_{1}\right)-F\left(x, s_{2}\right)\right)\left(s_{1}-s_{2}\right) \geq c_{1}\left|s_{1}-s_{2}\right|^{p} ;
$$

(III) for every $s_{1}, s_{2} \in \mathbf{R}^{M}$ and for every $x \in \Omega$ we have

$$
\left|F\left(x, s_{1}\right)-F\left(x, s_{2}\right)\right| \leq c_{2}\left(\left|s_{1}\right|+\left|s_{2}\right|\right)^{p-1-\sigma}\left|s_{1}-s_{2}\right|^{\sigma} ;
$$

(IV) $F(x, 0)=0$ for every $x \in \Omega$.

As consequence of properties (III) and (IV) we have that the function $F$ also satisfies

(V) $|F(x, s)| \leq c_{2}|s|^{p-1}$ for every $s \in \mathbf{R}^{M}$ and for every $x \in \Omega$, and by properties (II) and (IV) we get

(VI) $F(x, s) s \geq c_{1}|s|^{p}$ for every $s \in \mathbf{R}^{M}$ and for every $x \in \Omega$.

In the following we shall fix a constant $L>0$ and we shall denote by $\mathcal{F}(L)$ the class $\mathcal{F}(\alpha, L, 1)$, where $\alpha$ is the positive constant which appears in condition (i) above.

From now on by $C$ we shall denote a positive constant, depending only on $\alpha, \beta$, $L$, and $p$, which can change from line to line.

Let $f \in W^{-1, p^{\prime}}\left(\Omega, \mathbf{R}^{M}\right)$, let $\left(\mu_{n}\right)$ be a sequence of $\mathcal{M}_{0}^{p}(\Omega)$, and let $F_{n} \in \mathcal{F}(L)$. Let us consider the following nonlinear systems with boundary Dirichlet condition:

$$
\left\{\begin{array}{l}
u_{n} \in W_{0}^{1, p}\left(\Omega, \mathbf{R}^{M}\right) \cap L_{\mu_{n}}^{p}\left(\Omega, \mathbf{R}^{M}\right), \\
\int_{\Omega} a\left(x, D u_{n}\right) D v d x+\int_{\Omega} F_{n}\left(x, u_{n}\right) v d \mu_{n}=\langle f, v\rangle \\
\forall v \in W_{0}^{1, p}\left(\Omega, \mathbf{R}^{M}\right) \cap L_{\mu_{n}}^{p}\left(\Omega, \mathbf{R}^{M}\right) .
\end{array}\right.
$$

Since by Remark $3.1\langle f, \cdot\rangle$ is a functional in $\left(W_{0}^{1, p}\left(\Omega, \mathbf{R}^{M}\right) \cap L_{\mu_{n}}^{p}\left(\Omega, \mathbf{R}^{M}\right)\right)^{\prime}$, by assumptions (i)-(v) and (I)-(VI) the theory of monotone operators (see [23]) assures the existence of a unique solution $u_{n}$ of problem (5.1). From (v) and (VI), taking $u_{n}$ as a test function in (5.1), it is easy to see that the sequence $\left(u_{n}\right)$ is bounded in $W_{0}^{1, p}\left(\Omega, \mathbf{R}^{M}\right)$ for any choice of $\left(\mu_{n}\right)$ and $\left(F_{n}\right)$. Thus, up to a subsequence, the sequence $\left(u_{n}\right)$ converges weakly in $W_{0}^{1, p}\left(\Omega, \mathbf{R}^{M}\right)$ to some function $u \in W_{0}^{1, p}\left(\Omega, \mathbf{R}^{M}\right)$. Our goal is to find the variational problem satisfied by the function $u$. To this aim we shall consider special sequences of test functions $v_{n} \in W_{0}^{1, p}\left(\Omega, \mathbf{R}^{M}\right) \cap L_{\mu_{n}}^{p}\left(\Omega, \mathbf{R}^{M}\right)$ which converge weakly to some function $v \in W_{0}^{1, p}\left(\Omega, \mathbf{R}^{M}\right) \cap L_{\mu}^{p}\left(\Omega, \mathbf{R}^{M}\right)$, and we shall try to take the limit in problem (5.1). This is the energy method of L. Tartar.

In order to prove that the structure of the limit problem is local (i.e., it does not depend on the choice of the domain $\Omega$ and of the boundary data), in what follows, we shall consider a more general situation. Namely, we shall study the asymptotic behavior of an arbitrary sequence $\left(u_{n}\right)$ of solutions of the problems

$$
\left\{\begin{array}{l}
u_{n} \in W^{1, p}\left(\Omega^{\prime}, \mathbf{R}^{M}\right) \cap L_{\mu_{n}}^{p}\left(\Omega^{\prime}, \mathbf{R}^{M}\right), \\
\int_{\Omega^{\prime}} a\left(x, D u_{n}\right) D v d x+\int_{\Omega^{\prime}} F_{n}\left(x, u_{n}\right) v d \mu_{n}=\left\langle f_{n}, v\right\rangle \\
\forall v \in W_{0}^{1, p}\left(\Omega^{\prime}, \mathbf{R}^{M}\right) \cap L_{\mu_{n}}^{p}\left(\Omega^{\prime}, \mathbf{R}^{M}\right),
\end{array}\right.
$$

where $\Omega^{\prime}$ is an open subset of $\Omega, f_{n} \in\left(W_{0}^{1, p}\left(\Omega^{\prime}, \mathbf{R}^{M}\right) \cap L_{\mu_{n}}^{p}\left(\Omega^{\prime}, \mathbf{R}^{M}\right)\right)^{\prime}$. We do not require any boundary data for $u_{n}$, while we assume that the sequence $\left(u_{n}\right)$ is bounded 
in $W^{1, p}\left(\Omega^{\prime}, \mathbf{R}^{M}\right)$, which implies in particular that, up to a subsequence, $\left(u_{n}\right)$ converges weakly to some $u$ in $W^{1, p}\left(\Omega^{\prime}, \mathbf{R}^{M}\right)$. For the sequence $\left(f_{n}\right)$, we shall assume a notion of convergence specified by the following definition.

Definition 5.1. Let $\left(\mu_{n}\right)$ be a sequence of $\mathcal{M}_{0}^{p}(\Omega)$ which $\gamma^{-\Delta_{p}}$-converges to a measure $\mu$. Let $\left(f_{n}\right)$ be a sequence of functionals, with $f_{n} \in\left(W_{0}^{1, p}\left(\Omega^{\prime}, \mathbf{R}^{M}\right) \cap\right.$ $\left.L_{\mu_{n}}^{p}\left(\Omega^{\prime}, \mathbf{R}^{M}\right)\right)^{\prime}$, and let $f \in\left(W_{0}^{1, p}\left(\Omega^{\prime}, \mathbf{R}^{M}\right) \cap L_{\mu}^{p}\left(\Omega^{\prime}, \mathbf{R}^{M}\right)\right)^{\prime}$. We shall say that the sequence $\left(f_{n}\right)$ converges to $f$ in the sense of $\left(\mathcal{H}_{\Omega^{\prime}}\right)$ if the following condition is satisfied:

$\left(\mathcal{H}_{\Omega^{\prime}}\right)$ If $v \in W_{0}^{1, p}\left(\Omega^{\prime}, \mathbf{R}^{M}\right) \cap L_{\mu}^{p}\left(\Omega^{\prime}, \mathbf{R}^{M}\right), v_{n} \in W_{0}^{1, p}\left(\Omega^{\prime}, \mathbf{R}^{M}\right) \cap L_{\mu_{n}}^{p}\left(\Omega^{\prime}, \mathbf{R}^{M}\right)$ for every $n$, and $\left(v_{n}\right)$ converges to $v$ weakly in $W_{0}^{1, p}\left(\Omega^{\prime}, \mathbf{R}^{M}\right)$, then $\left\langle f_{n}, v_{n}\right\rangle \rightarrow$ $\langle f, v\rangle$.

The next lemma gives an estimate of the norm in $\left(W_{0}^{1, p}\left(\Omega^{\prime}, \mathbf{R}^{M}\right) \cap L_{\mu_{n}}^{p}\left(\Omega^{\prime}, \mathbf{R}^{M}\right)\right)^{\prime}$ of a sequence of functionals $\left(f_{n}\right)$ which converges in the sense of $\left(\mathcal{H}_{\Omega^{\prime}}\right)$, while Proposition 5.3 gives a local estimate of the norm in $L_{\mu_{n}}^{p}\left(\Omega^{\prime}, \mathbf{R}^{M}\right)$ of the corresponding solutions $u_{n}$ of problem (5.2).

LEMmA 5.2. Let $f \in\left(W_{0}^{1, p}\left(\Omega^{\prime}, \mathbf{R}^{M}\right) \cap L_{\mu}^{p}\left(\Omega^{\prime}, \mathbf{R}^{M}\right)\right)^{\prime}$, and let $f_{n} \in\left(W_{0}^{1, p}\left(\Omega^{\prime}, \mathbf{R}^{M}\right) \cap\right.$ $\left.L_{\mu_{n}}^{p}\left(\Omega^{\prime}, \mathbf{R}^{M}\right)\right)^{\prime}$ for every $n$. If $\left(f_{n}\right)$ converges to $f$ in the sense of $\left(\mathcal{H}_{\Omega^{\prime}}\right)$, then $\left(\left\|f_{n}\right\|\right)$ converges to $\|f\|$, where the norm of $f_{n}$ (resp., $\left.f\right)$ is taken in the space $\left(W_{0}^{1, p}\left(\Omega^{\prime}, \mathbf{R}^{M}\right) \cap L_{\mu_{n}}^{p}\left(\Omega^{\prime}, \mathbf{R}^{M}\right)\right)^{\prime}$ (resp., $\left.\left(W_{0}^{1, p}\left(\Omega^{\prime}, \mathbf{R}^{M}\right) \cap L_{\mu}^{p}\left(\Omega^{\prime}, \mathbf{R}^{M}\right)\right)^{\prime}\right)$.

Proof. Let $\left(\zeta_{n}\right)$ be a sequence such that $\zeta_{n} \in W_{0}^{1, p}\left(\Omega^{\prime}, \mathbf{R}^{M}\right) \cap L_{\mu_{n}}^{p}\left(\Omega^{\prime}, \mathbf{R}^{M}\right)$, with unit norm and $\left\|f_{n}\right\|=\left\langle f_{n}, \zeta_{n}\right\rangle$. Then, up to a subsequence, $\left(\zeta_{n}\right)$ converges weakly in $W_{0}^{1, p}\left(\Omega^{\prime}, \mathbf{R}^{M}\right)$ to some function $\zeta$, by Theorem $4.4 \zeta \in W_{0}^{1, p}\left(\Omega^{\prime}, \mathbf{R}^{M}\right) \cap L_{\mu}^{p}\left(\Omega^{\prime}, \mathbf{R}^{M}\right)$, and $\|\zeta\| \leq 1$. Since $\left(f_{n}\right)$ converges in the sense of $\left(\mathcal{H}_{\Omega^{\prime}}\right)$ we have that

$$
\lim _{n \rightarrow \infty}\left\|f_{n}\right\|=\lim _{n \rightarrow \infty}\left\langle f_{n}, \zeta_{n}\right\rangle=\langle f, \zeta\rangle \leq\|f\| .
$$

In order to prove the opposite inequality let us consider the function $\zeta$ such that $\zeta \in W_{0}^{1, p}\left(\Omega^{\prime}, \mathbf{R}^{M}\right) \cap L_{\mu}^{p}\left(\Omega^{\prime}, \mathbf{R}^{M}\right)$, with unit norm, $\|f\|=\langle f, \zeta\rangle$, and let $\left(\psi_{m}\right)$ be a sequence in $C_{0}^{\infty}\left(\Omega^{\prime}, \mathbf{R}^{M}\right)$ such that $\left(w \psi_{m}\right)$ converges strongly to $\zeta$ in $W_{0}^{1, p}\left(\Omega^{\prime}, \mathbf{R}^{M}\right) \cap$ $L_{\mu}^{p}\left(\Omega^{\prime}, \mathbf{R}^{M}\right)$. By Lemma 4.2 we have that the norm in the space $W_{0}^{1, p}\left(\Omega^{\prime}, \mathbf{R}^{M}\right) \cap$ $L_{\mu_{n}}^{p}\left(\Omega^{\prime}, \mathbf{R}^{M}\right)$ of the functions $w_{n} \psi_{m}$ converges to the norm of $w \psi_{m}$ in the space $W_{0}^{1, p}\left(\Omega^{\prime}, \mathbf{R}^{M}\right) \cap L_{\mu}^{p}\left(\Omega^{\prime}, \mathbf{R}^{M}\right)$. Thus, since $\left(w_{n} \psi_{m}\right)$ converges weakly in $W_{0}^{1, p}\left(\Omega^{\prime}, \mathbf{R}^{M}\right)$ to $w \psi_{m}$, we have

$$
\begin{gathered}
\|f\|=\langle f, \zeta\rangle=\lim _{m \rightarrow \infty} \lim _{n \rightarrow \infty}\left\langle f_{n}, w_{n} \psi_{m}\right\rangle \\
\leq \lim _{m \rightarrow \infty} \liminf _{n \rightarrow \infty}\left\|f_{n}\right\|\left\|w_{n} \psi_{m}\right\|=\liminf _{n \rightarrow \infty}\left\|f_{n}\right\|\|\zeta\|=\liminf _{n \rightarrow \infty}\left\|f_{n}\right\| .
\end{gathered}
$$

Proposition 5.3. Let $\left(u_{n}\right)$ be a sequence of solutions of problems (5.2). If the sequence $\left(u_{n}\right)$ is bounded in $W^{1, p}\left(\Omega^{\prime}, \mathbf{R}^{M}\right)$, then

$$
\int_{\Omega^{\prime}}\left|u_{n}\right|^{p} \varphi d \mu_{n} \leq M
$$

for every $\varphi \in C_{0}^{1}\left(\Omega^{\prime}\right)$, with $\varphi \geq 0$, where the constant $M$ depends on the norm in $C_{0}^{1}\left(\Omega^{\prime}\right)$ of $\varphi$.

Proof. The proof follows immediately, taking $u_{n} \varphi$ as test function in (5.2), by Lemma 5.2 and conditions (v) and (VI).

The following proposition shows that, without any additional assumption, the sequence $\left(u_{n}\right)$ converges strongly in $W^{1, r}\left(\Omega^{\prime}, \mathbf{R}^{M}\right)$ for every $1 \leq r<p$. 
Proposition 5.4. Let $\left(u_{n}\right)$, with $u_{n} \in W^{1, p}\left(\Omega^{\prime}, \mathbf{R}^{M}\right) \cap L_{\mu_{n}}^{p}\left(\Omega^{\prime}, \mathbf{R}^{M}\right)$, be a sequence which converges to some function $u$ weakly in $W^{1, p}\left(\Omega^{\prime}, \mathbf{R}^{M}\right)$. Suppose that there exists a sequence $\left(f_{n}\right)$, with $f_{n} \in\left(W_{0}^{1, p}\left(\Omega^{\prime}, \mathbf{R}^{M}\right) \cap L_{\mu_{n}}^{p}\left(\Omega^{\prime}, \mathbf{R}^{M}\right)\right)^{\prime}$, which converges to $f \in\left(W_{0}^{1, p}\left(\Omega^{\prime}, \mathbf{R}^{M}\right) \cap L_{\mu}^{p}\left(\Omega^{\prime}, \mathbf{R}^{M}\right)\right)^{\prime}$ in the sense of $\left(\mathcal{H}_{\Omega^{\prime}}\right)$, such that $u_{n}$ satisfies problem (5.2). Then $\left(u_{n}\right)$ converges to u strongly in $W^{1, r}\left(\Omega^{\prime}, \mathbf{R}^{M}\right)$ for every $r<p$, and hence a subsequence of $\left(D u_{n}\right)$ converges to Du pointwise a.e. in $\Omega^{\prime}$.

Proof. The proof follows the lines of the one given in [16] (see also [1]).

In the course this proof we shall denote by $C$ a positive constant independent on $n$. Let $\Psi: \mathbf{R} \mapsto \mathbf{R}$ be a $C^{1}$ function which satisfies the following properties:

$$
\begin{aligned}
& \Psi(t)=1 \quad \text { if }|t|<1, \quad \Psi(t)=0 \quad \text { if } \quad|t| \geq 2, \\
& |\Psi(t)| \leq 1 \quad \forall t \in \mathbf{R}, \quad\left|\Psi^{\prime}(t)\right| \leq 2 \quad \forall t \in \mathbf{R},
\end{aligned}
$$

and let $\Phi(y)=\Psi(|y|) y$. Let $\delta>0$ and, for every $n \in \mathbf{N}$, let $\delta_{n} \leq \delta$ be a positive real number that we shall fix later. For every such a $\delta_{n}$ we define the function $\Phi_{\delta_{n}}(y)=$ $\delta_{n} \Phi\left(y / \delta_{n}\right)$. Given $\varphi \in C_{0}^{1}\left(\Omega^{\prime}\right)$, with $\varphi \geq 0$, we can take $\left(\Phi_{\delta_{n}}\left(u_{n}\right)+w_{n} \Phi_{\delta_{n}}\left(u_{n}-u\right)\right) \varphi$ as test function in problem (5.2), and we obtain

$$
\begin{gathered}
\int_{\Omega^{\prime}} a\left(x, D u_{n}\right) D \Phi_{\delta_{n}}\left(u_{n}\right) D u_{n} \varphi d x \\
+\int_{\Omega^{\prime}}\left(a\left(x, D u_{n}\right)-a(x, D u)\right) D \Phi_{\delta_{n}}\left(u_{n}-u\right) D\left(u_{n}-u\right) w_{n} \varphi d x \\
+\int_{\Omega^{\prime}} a\left(x, D u_{n}\right)\left(\Phi_{\delta_{n}}\left(u_{n}\right) \otimes D \varphi+\Phi_{\delta_{n}}\left(u_{n}-u\right) \otimes D\left(w_{n} \varphi\right)\right) d x \\
+\int_{\Omega^{\prime}} F_{n}\left(x, u_{n}\right)\left(w_{n} \Phi_{\delta_{n}}\left(u_{n}-u\right)+\Phi_{\delta_{n}}\left(u_{n}\right)\right) \varphi d \mu_{n} \\
=\left\langle f_{n},\left(w_{n} \Phi_{\delta_{n}}\left(u_{n}-u\right)+\Phi_{\delta_{n}}\left(u_{n}\right)\right) \varphi\right\rangle \\
-\int_{\Omega^{\prime}} a(x, D u) D \Phi_{\delta_{n}}\left(u_{n}-u\right) D\left(u_{n}-u\right) w_{n} \varphi d x .
\end{gathered}
$$

Since $\left(u_{n}\right)$ is bounded in $W^{1, p}\left(\Omega^{\prime}, \mathbf{R}^{M}\right),\left(w_{n}\right)$ is bounded in $W^{1, p}(\Omega)$, and $\left|\Phi_{\delta_{n}}\right| \leq$ $2 \delta_{n} \leq 2 \delta$, by condition (iv), we have

$$
\left|\int_{\Omega^{\prime}} a\left(x, D u_{n}\right)\left(\Phi_{\delta_{n}}\left(u_{n}\right) \otimes D \varphi+\Phi_{\delta_{n}}\left(u_{n}-u\right) \otimes D\left(w_{n} \varphi\right)\right) d x\right| \leq C \delta .
$$

From property (V), Hölder's inequality, and Proposition 5.3 it follows that

$$
\left|\int_{\Omega^{\prime}} F_{n}\left(x, u_{n}\right) \Phi_{\delta_{n}}\left(u_{n}-u\right) w_{n} \varphi d \mu_{n}\right| \leq C \delta,
$$

while from property (VI) and the definition of the function $\Phi$ we get

$$
\int_{\Omega^{\prime}} F_{n}\left(x, u_{n}\right) \Phi_{\delta_{n}}\left(u_{n}\right) \varphi d \mu_{n} \geq 0
$$

Since $\left(\Phi_{\delta_{n}}\left(u_{n}-u\right) w_{n} \varphi\right)$ converges weakly to zero in $W_{0}^{1, p}\left(\Omega, \mathbf{R}^{M}\right)$ and $\left(f_{n}\right)$ converges in the sense of $\left(\mathcal{H}_{\Omega^{\prime}}\right)$, we have

$$
\left\langle f_{n}, w_{n} \Phi_{\delta_{n}}\left(u_{n}-u\right) \varphi\right\rangle=o_{n} .
$$


Moreover, as $0<\delta_{n} \leq \delta$ and the sequence $\left(\Phi_{\delta_{n}}\right)$ is uniformly Lipschitz, it is easy to see that there exists a positive number $\tilde{\delta} \leq \delta$ such that

$$
\limsup _{n \rightarrow \infty}\left\langle f_{n}, \Phi_{\delta_{n}}\left(u_{n}\right) \varphi\right\rangle=\left\langle f, \Phi_{\tilde{\delta}}(u) \varphi\right\rangle .
$$

Finally, since $\left(D \Phi_{\delta_{n}}\left(u_{n}-u\right) D\left(u_{n}-u\right)\right)$ converges weakly to zero in $L^{p}\left(\Omega^{\prime}, \mathbf{R}^{M}\right)$ we also obtain that

$$
\int_{\Omega^{\prime}} a(x, D u) D \Phi_{\delta_{n}}\left(u_{n}-u\right) D\left(u_{n}-u\right) w_{n} \varphi d x=o_{n} .
$$

Thus, by assumptions (i)-(v), by (5.4)-(5.10), and by the definition of the function $\Phi$, we get

$$
\begin{aligned}
& \int_{\left\{\left|u_{n}\right|<\delta_{n}\right\}_{\Omega^{\prime}}}\left|D u_{n}\right|^{p} \varphi d x,+\int_{\left\{\left|u_{n}-u\right|<\delta_{n}\right\}_{\Omega^{\prime}}}\left|D\left(u_{n}-u\right)\right|^{p} w_{n} \varphi d x \\
\leq & C \int_{\left\{\delta_{n} \leq\left|u_{n}-u\right|<2 \delta_{n}\right\}_{\Omega^{\prime}}}\left(h+|D u|+\left|D u_{n}\right|\right)^{p-2}\left|D\left(u_{n}-u\right)\right|^{2} d x \\
+ & C \int_{\left\{\delta_{n} \leq\left|u_{n}\right|<2 \delta_{n}\right\}_{\Omega^{\prime}}}\left(k+\eta\left|D u_{n}\right|^{p-1}\right)\left|D u_{n}\right| d x+\left\langle f, \Phi_{\tilde{\delta}}(u) \varphi\right\rangle+C \delta+o_{n},
\end{aligned}
$$

where we also used the fact that the sequence $\left(w_{n}\right)$ is bounded in $L^{\infty}(\Omega)$. Now, since $\left(u_{n}\right)$ is bounded in $W^{1, p}\left(\Omega^{\prime}, \mathbf{R}^{M}\right)$, there exists a positive constant $K$ such that

$$
\int_{\Omega^{\prime}}\left(h+|D u|+\left|D u_{n}\right|\right)^{p-2}\left|D\left(u_{n}-u\right)\right|^{2} d x+\int_{\Omega^{\prime}}\left(k+\eta\left|D u_{n}\right|^{p-1}\right)\left|D u_{n}\right| d x \leq K .
$$

In particular, if we fix $J \in \mathbf{N}$ and $\gamma>0$, then we have

$$
\begin{aligned}
& \sum_{j=1}^{J}\left(\int_{\left\{2^{j-1} \gamma \leq\left|u_{n}-u\right|<2^{j} \gamma\right\}_{\Omega^{\prime}}}\left(h+|D u|+\left|D u_{n}\right|\right)^{p-2}\left|D\left(u_{n}-u\right)\right|^{2} d x\right. \\
& \left.\quad+\int_{\left\{2^{j-1} \gamma \leq\left|u_{n}\right|<2^{j} \gamma\right\}_{\Omega^{\prime}}}\left(k+\eta\left|D u_{n}\right|^{p-1}\right)\left|D u_{n}\right| d x\right) \leq K
\end{aligned}
$$

so that, for every $n \in \mathbf{N}$, there exists $j(n) \in\{1, \ldots, J\}$ such that

$$
\begin{gathered}
\int_{\left\{2^{j(n)-1} \gamma \leq\left|u_{n}-u\right|<2^{j(n)} \gamma\right\}_{\Omega^{\prime}}}\left(h+|D u|+\left|D u_{n}\right|\right)^{p-2}\left|D\left(u_{n}-u\right)\right|^{2} d x \\
+\int_{\left\{2^{j(n)-1} \gamma \leq\left|u_{n}\right|<2^{j(n)} \gamma\right\}_{\Omega^{\prime}}}\left(k+\eta\left|D u_{n}\right|^{p-1}\right)\left|D u_{n}\right| d x \leq \frac{K}{J} .
\end{gathered}
$$

If in (5.11) we take $\delta=2^{J} \gamma$ and $\delta_{n}=2^{j(n)-1} \gamma$, then we get

$$
\begin{gathered}
\int_{\left\{\left|u_{n}\right|<\gamma\right\}_{\Omega^{\prime}}}\left|D u_{n}\right|^{p} \varphi d x+\int_{\left\{\left|u_{n}-u\right|<\gamma\right\}_{\Omega^{\prime}}}\left|D\left(u_{n}-u\right)\right|^{p} w_{n} \varphi d x \\
\leq \frac{C}{J}+C 2^{J} \gamma+\left\langle f, \Phi_{\tilde{\delta}}(u) \varphi\right\rangle+o_{n},
\end{gathered}
$$

where we used the fact that $\delta_{n} \geq \gamma$ for every $n \in \mathbf{N}$. By Rellich's theorem the sequence $\left(u_{n}\right)$ converges to $u$ strongly in $L_{\text {loc }}^{p}\left(\Omega^{\prime}, \mathbf{R}^{M}\right)$, and hence, up to a subsequence, 
pointwise a.e. in $\Omega^{\prime}$. Thus, by Egorov's theorem, for every $\sigma>0$ there exists a subset $S$ of $\Omega^{\prime}$, with $|S|<\sigma$, such that $\left(u_{n}\right)$ converges to $u$ uniformly on $\Omega^{\prime} \backslash S$.

Now let $\varepsilon>0$. If we choose $J \in \mathbf{N}$ and $\gamma>0$ such that $1 / J<\varepsilon$ and $\delta=2^{J} \gamma=\varepsilon$, then by (5.12) we have

$\limsup _{n \rightarrow \infty}\left(\int_{\left\{\left|u_{n}\right|<\gamma\right\}_{\Omega^{\prime}}}\left|D u_{n}\right|^{p} \varphi d x+\int_{\left\{\left|u_{n}-u\right|<\gamma\right\}_{\Omega^{\prime}}}\left|D\left(u_{n}-u\right)\right|^{p} w_{n} \varphi d x\right) \leq C \varepsilon+\left\langle f, \Phi_{\tilde{\delta}}(u) \varphi\right\rangle$.

Moreover, for $n$ large enough we have that $\Omega^{\prime} \backslash S \subseteq\left\{\left|u_{n}-u\right|<\gamma\right\}_{\Omega^{\prime}}$ and $\{u=$ $0\}_{\Omega^{\prime}} \backslash S \subseteq\left\{\left|u_{n}\right|<\gamma\right\}_{\Omega^{\prime}}$, so that we get

$$
\begin{aligned}
& \limsup _{n \rightarrow \infty}\left(\int_{\{u=0\}_{\Omega^{\prime}} \backslash S}\left|D u_{n}\right|^{p} \varphi d x+\int_{\Omega^{\prime} \backslash S}\left|D\left(u_{n}-u\right)\right|^{p} w_{n} \varphi d x\right) \\
& \quad \leq C \varepsilon+\left\langle f, \Phi_{\tilde{\delta}}(u) \varphi\right\rangle,
\end{aligned}
$$

which, by using that $0 \leq \tilde{\delta} \leq \delta=\varepsilon$ and $\Phi_{\tilde{\delta}}(u) \varphi$ converges strongly to zero in $W_{0}^{1, p}\left(\Omega, \mathbf{R}^{M}\right) \cap L_{\mu}^{p}\left(\Omega, \mathbf{R}^{M}\right)$ as $\varepsilon$ tends to zero, gives

$$
\lim _{n \rightarrow \infty}\left(\int_{\{u=0\}_{\Omega^{\prime}} \backslash S}\left|D u_{n}\right|^{p} \varphi d x+\int_{\Omega^{\prime} \backslash S}\left|D\left(u_{n}-u\right)\right|^{p} w_{n} \varphi d x\right)=0 .
$$

By the arbitrariness of $\sigma$, we get, up to a subsequence, that $\left(D\left(u_{n}-u\right) w_{n}\right)$ and $\left(D u_{n} 1_{\{|u|=0\}_{\Omega^{\prime}}}\right)$ converges to zero pointwise a.e. in $\Omega^{\prime}$. Moreover, since $\left(w_{n}\right)$ converges to $w$ strongly in $L^{p}\left(\Omega^{\prime}, \mathbf{R}^{M}\right)$ and by Lemma $4.5\{w>0\} \supseteq\{|u|>0\}_{\Omega^{\prime}}$, this implies that $\left(D u_{n}\right)$ converges pointwise to $D u$ a.e. in $\{|u|>0\}_{\Omega^{\prime}}$ and hence, as $|D u|=0$ a.e. in $\{|u|=0\}_{\Omega^{\prime}},\left(D u_{n}\right)$ converges pointwise to $D u$ a.e. in $\Omega^{\prime}$.

Finally, since $\left(u_{n}\right)$ is bounded in $W^{1, p}\left(\Omega^{\prime}, \mathbf{R}^{M}\right)$, we obtain that $\left(u_{n}\right)$ converges to $u$ strongly in $W^{1, r}\left(\Omega^{\prime}, \mathbf{R}^{M}\right)$ for every $r<p$.

Remark 5.5. Under the same assumptions of Proposition 5.4, by (v) and Proposition 5.4 we have that $\left(a\left(x, D u_{n}\right)\right)$ converges to $a(x, D u)$ weakly in $L^{p^{\prime}}\left(\Omega^{\prime}, \mathbf{M}^{M \times N}\right)$ and strongly in $L^{r}\left(\Omega^{\prime}, \mathbf{M}^{M \times N}\right)$ for every $1 \leq r<p^{\prime}$. Similarly we deduce that $\left(a\left(x, D\left(u_{n}-\right.\right.\right.$ $u))$ ) converges to zero weakly in $L^{p^{\prime}}\left(\Omega^{\prime}, \mathbf{M}^{M \times N}\right)$ and strongly in $L^{r}\left(\Omega^{\prime}, \mathbf{M}^{M \times N}\right)$ for every $1 \leq r<p^{\prime}$.

6. The limit problem. In this section we shall prove the main result of this paper (Theorem 6.4). We shall consider a sequence $\left(u_{n}\right)$ of solutions of problems (5.2), with $F_{n} \in \mathcal{F}(L)$ and $\mu_{n} \in \mathcal{M}_{0}^{p}(\Omega)$, which satisfies

$$
\int_{\Omega^{\prime}}\left|D u_{n}\right|^{p} d x+\int_{\Omega^{\prime}}\left|u_{n}\right|^{p} d \mu_{n} \leq M
$$

where $M$ is a positive constant which depends on the sequence $\left(u_{n}\right)$. We shall show that a cluster point $u$ of such a sequence is a solution of a variational problem similar to (5.2). Namely we shall prove that the limit problem will be of the form

$$
\left\{\begin{array}{l}
u \in W^{1, p}\left(\Omega^{\prime}, \mathbf{R}^{M}\right) \cap L_{\mu}^{p}\left(\Omega^{\prime}, \mathbf{R}^{M}\right), \\
\int_{\Omega^{\prime}} a(x, D u) D v d x+\int_{\Omega^{\prime}} F(x, u) v d \mu=\langle f, v\rangle \\
\forall v \in W_{0}^{1, p}\left(\Omega^{\prime}, \mathbf{R}^{M}\right) \cap L_{\mu}^{p}\left(\Omega^{\prime}, \mathbf{R}^{M}\right),
\end{array}\right.
$$

where $\mu$ is a measure in $\mathcal{M}_{0}^{p}(\Omega)$ and $F(x, s)$ is a vector function in $\mathcal{F}(\alpha, C, 1 /(p-1))$ for a constant $C$ which depends only on $\alpha, \beta, L, N$, and $p$. 
Remark 6.1. Let $\mu \in \mathcal{M}_{0}^{p}(\Omega)$ and let $\tilde{F}, F \in \mathcal{F}\left(c_{1}, c_{2}, \sigma\right)$ be two functions such that for every $s \in \mathbf{R}^{M} F(x, s)=\tilde{F}(x, s) \mu$-a.e. in $\{w>0\}$, where $w$ is the solution of problem (3.3). If in problem (6.2) we change $F$ by $\tilde{F}$ we obtain an equivalent problem. In particular the function $F(x, s)$ can be defined arbitrarily in the set $\{w=0\}$.

Let us introduce now a notion of convergence in the space $\mathcal{M}_{0}^{p}(\Omega) \times \mathcal{F}\left(c_{1}, c_{2}, \sigma\right)$, with $c_{1}>0, c_{2}>0$, and $0<\sigma \leq 1$.

Definition 6.2. Let $\left(\mu_{n}\right)$ be a sequence in $\mathcal{M}_{0}^{p}(\Omega)$, let $\left(F_{n}\right)$ be a sequence in $\mathcal{F}\left(c_{1}, c_{2}, \sigma\right)$, let $\mu \in \mathcal{M}_{0}^{p}(\Omega)$ and $F \in \mathcal{F}\left(c_{1}, c_{2}, \sigma\right)$. We say that the pairs $\left(\mu_{n}, F_{n}\right) \gamma^{A}$ converge (in $\Omega$ ) to the pair $(\mu, F)$ if the following property holds: for any open set $\Omega^{\prime} \subseteq$ $\Omega$, for any sequence of functionals $\left(f_{n}\right)$ with $f_{n} \in\left(W_{0}^{1, p}\left(\Omega^{\prime}, \mathbf{R}^{M}\right) \cap L_{\mu_{n}}^{p}\left(\Omega^{\prime}, \mathbf{R}^{M}\right)\right)^{\prime}$, which converges to some $f \in\left(W_{0}^{1, p}\left(\Omega^{\prime}, \mathbf{R}^{M}\right) \cap L_{\mu}^{p}\left(\Omega^{\prime}, \mathbf{R}^{M}\right)\right)^{\prime}$ in the sense of $\left(\mathcal{H}_{\Omega^{\prime}}\right)$ (according with Definition 5.1), and for any sequence $\left(u_{n}\right)$ of solutions of problems (5.2) satisfying (6.1), all cluster points of the sequence $\left(u_{n}\right)$ in the weak topology of $W^{1, p}\left(\Omega^{\prime}, \mathbf{R}^{M}\right)$ satisfy problem (6.2).

The most important property of the $\gamma^{A}$ convergence is the following result.

Proposition 6.3. Let $\left(\left(\mu_{n}, F_{n}\right)\right)$ be a sequence in $\mathcal{M}_{0}^{p}(\Omega) \times \mathcal{F}\left(c_{1}, c_{2}, \sigma\right)$ which $\gamma^{A}$-converges to a pair $(\mu, F)$. Then for any open set $\Omega^{\prime} \subseteq \Omega$ and for any sequence $\left(f_{n}\right)$, with $f_{n} \in\left(W_{0}^{1, p}\left(\Omega^{\prime}, \mathbf{R}^{M}\right) \cap L_{\mu_{n}}^{p}\left(\Omega^{\prime}, \mathbf{R}^{M}\right)\right)^{\prime}$, which converges to some $f \in\left(W_{0}^{1, p}\left(\Omega^{\prime}, \mathbf{R}^{M}\right) \cap L_{\mu}^{p}\left(\Omega^{\prime}, \mathbf{R}^{M}\right)\right)^{\prime}$ in the sense of $\left(\mathcal{H}_{\Omega^{\prime}}\right)$, the unique solution $u_{n}$ of the problem

$$
\left\{\begin{array}{l}
u_{n} \in W_{0}^{1, p}\left(\Omega^{\prime}, \mathbf{R}^{M}\right) \cap L_{\mu_{n}}^{p}\left(\Omega^{\prime}, \mathbf{R}^{M}\right), \\
\int_{\Omega^{\prime}} a\left(x, D u_{n}\right) D v d x+\int_{\Omega^{\prime}} F_{n}\left(x, u_{n}\right) v d \mu_{n}=\left\langle f_{n}, v\right\rangle \\
\forall v \in W_{0}^{1, p}\left(\Omega^{\prime}, \mathbf{R}^{M}\right) \cap L_{\mu_{n}}^{p}\left(\Omega^{\prime}, \mathbf{R}^{M}\right)
\end{array}\right.
$$

converges weakly in $W_{0}^{1, p}\left(\Omega^{\prime}, \mathbf{R}^{M}\right)$ to the unique solution $u$ of the problem

$$
\left\{\begin{array}{l}
u \in W_{0}^{1, p}\left(\Omega^{\prime}, \mathbf{R}^{M}\right) \cap L_{\mu}^{p}\left(\Omega^{\prime}, \mathbf{R}^{M}\right), \\
\int_{\Omega^{\prime}} a(x, D u) D v d x+\int_{\Omega^{\prime}} F(x, u) v d \mu=\langle f, v\rangle \\
\forall v \in W_{0}^{1, p}\left(\Omega^{\prime}, \mathbf{R}^{M}\right) \cap L_{\mu}^{p}\left(\Omega^{\prime}, \mathbf{R}^{M}\right) .
\end{array}\right.
$$

Proof. By using $u_{n}$ as a test function in (6.3) and by taking into account Lemma 5.2 , we deduce that the sequence $\left(u_{n}\right)$ satisfies (6.1). This implies in particular that there exists a subsequence of $\left(u_{n}\right)$ which converges weakly in $W_{0}^{1, p}\left(\Omega^{\prime}, \mathbf{R}^{M}\right)$ to a function $u \in W_{0}^{1, p}\left(\Omega^{\prime}, \mathbf{R}^{M}\right)$. By the definition of $\gamma^{A}$-convergence, the function $u$ satisfies (6.4). Since this problem has a unique solution, the whole sequence $\left(u_{n}\right)$ converges to $u$.

The following theorem gives a compactness result for the $\gamma^{A}$-convergence.

THEOREM 6.4. Let $\left(\mu_{n}\right)$ be a sequence of measures in $\mathcal{M}_{0}^{p}(\Omega)$ and let $\left(F_{n}\right)$ be a sequence in $\mathcal{F}(L)$, with $L>0$. Then there exist an increasing sequence of integers $\left(n_{j}\right)$, a measure $\mu \in \mathcal{M}_{0}^{p}(\Omega)$, and a function $F \in \mathcal{F}(\alpha, C, 1 /(p-1))$ such that the pairs $\left(\mu_{n_{j}}, F_{n_{j}}\right) \gamma^{A}$-converge to $(\mu, F)$ in $\Omega$ (according to Definition 6.2), where $C$ is a positive constant which depends only on $\alpha, \beta, L, N$, and $p$.

Remark 6.5. The compactness result stated in Theorem 6.4 can be proved under more general assumptions on $\left(F_{n}\right)$. Namely, if the sequence $\left(F_{n}\right)$ belongs to 
$\mathcal{F}\left(c_{1}, c_{2}, \sigma\right)$, for some constants $c_{1}>0, c_{2}>0$, and $0<\sigma \leq 1$, then there exist an increasing sequence of integers $\left(n_{j}\right)$, a measure $\mu \in \mathcal{M}_{0}^{p}(\Omega)$, and a function $F \in \mathcal{F}\left(c_{1}^{\prime}, c_{2}^{\prime}, \sigma^{\prime}\right)$ such that the pairs $\left(\mu_{n_{j}}, F_{n_{j}}\right) \gamma^{A}$-converge to $(\mu, F)$ in $\Omega$. The positive constants $c_{1}$ and $c_{2}$ depend only on $\alpha, \beta, c_{1}, c_{2}, N, p$, and $\sigma$; while $\sigma^{\prime}=\min \{\sigma, 1 /(p-\sigma)\}$.

In order to simplify the exposition of the proof, we shall prove only the compactness result as stated in Theorem 6.4 (the proof of the general case stated in Remark 6.5 being analogous). Before proving Theorem 6.4 we need additional information on the behavior of the sequence $\left(u_{n}\right)$ of solutions of problems (5.2). To this aim we shall compare $\left(u_{n}\right)$ with the sequences $\left(w_{n} \psi_{m}\right), \psi_{m} \in C_{0}^{\infty}\left(\Omega, \mathbf{R}^{M}\right)$, of correctors for the $p$-Laplacian, studied in section 4.

In Lemma 6.6 and Propositions 6.7 and 6.8 , we shall consider an open set $\Omega^{\prime} \subseteq \Omega$, a sequence of measures $\left(\mu_{n}\right)$, a sequence of functions $\left(F_{n}\right)$, two sequences of functionals $\left(f_{n}\right),\left(g_{n}\right)$, two sequences of functions $\left(u_{n}\right),\left(z_{n}\right)$, a measure $\mu$, two functionals $f, g$, and two functions $u$ and $z$ such that

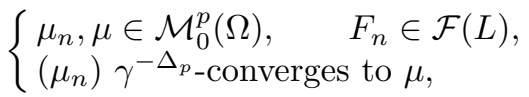

$$
\begin{aligned}
& \left\{\begin{array}{l}
f_{n}, g_{n} \in\left(W_{0}^{1, p}\left(\Omega^{\prime}, \mathbf{R}^{M}\right) \cap L_{\mu_{n}}^{p}\left(\Omega^{\prime}, \mathbf{R}^{M}\right)\right)^{\prime} \\
f, g \in\left(W_{0}^{1, p}\left(\Omega^{\prime}, \mathbf{R}^{M}\right) \cap L_{\mu}^{p}\left(\Omega^{\prime}, \mathbf{R}^{M}\right)\right)^{\prime} \\
f_{n} \rightarrow f \text { in the sense of }\left(\mathcal{H}_{\Omega^{\prime}}\right) \\
g_{n} \rightarrow g \text { in the sense of }\left(\mathcal{H}_{\Omega^{\prime}}\right)
\end{array}\right. \\
& \left\{\begin{array}{l}
u_{n}, z_{n} \in W^{1, p}\left(\Omega^{\prime}, \mathbf{R}^{M}\right) \cap L_{\mu_{n}}^{p}\left(\Omega^{\prime}, \mathbf{R}^{M}\right), \\
u, z \in W^{1, p}\left(\Omega^{\prime}, \mathbf{R}^{M}\right) \cap L_{\mu}^{p}\left(\Omega^{\prime}, \mathbf{R}^{M}\right), \\
u_{n} \rightarrow u \text { in } W^{1, p}\left(\Omega^{\prime}, \mathbf{R}^{M}\right), \\
z_{n} \rightarrow z \text { in } W^{1, p}\left(\Omega^{\prime}, \mathbf{R}^{M}\right),
\end{array}\right. \\
& \left\{\begin{array}{c}
\int_{\Omega^{\prime}} a\left(x, D u_{n}\right) D v d x+\int_{\Omega^{\prime}} F_{n}\left(x, u_{n}\right) v d \mu_{n}=\left\langle f_{n}, v\right\rangle \\
\forall v \in W_{0}^{1, p}\left(\Omega^{\prime}, \mathbf{R}^{M}\right) \cap L_{\mu_{n}}^{p}\left(\Omega^{\prime}, \mathbf{R}^{M}\right), \\
\int_{\Omega^{\prime}} a\left(x, D z_{n}\right) D v d x+\int_{\Omega^{\prime}} F_{n}\left(x, z_{n}\right) v d \mu_{n}=\left\langle f_{n}, v\right\rangle \\
\forall v \in W_{0}^{1, p}\left(\Omega^{\prime}, \mathbf{R}^{M}\right) \cap L_{\mu_{n}}^{p}\left(\Omega^{\prime}, \mathbf{R}^{M}\right) .
\end{array}\right.
\end{aligned}
$$

Lemma 6.6. Assume that (6.5), (6.6), (6.7), and (6.8) hold. Then for every function $\varphi \in C_{c}^{\infty}\left(\Omega^{\prime}\right)$, with $\varphi \geq 0$, we have the estimates

$$
\limsup _{n \rightarrow \infty}\left(\int_{\Omega^{\prime}}\left|D\left(u_{n}-u\right)\right|^{p} \varphi d x+\int_{\Omega^{\prime}}\left|u_{n}\right|^{p} \varphi d \mu_{n}\right) \leq C \int_{\Omega^{\prime}}|u|^{p} \varphi d \mu
$$

and

$$
\begin{gathered}
\limsup _{n \rightarrow \infty}\left(\int_{\Omega^{\prime}}\left|D\left(\left(u_{n}-z_{n}\right)-(u-z)\right)\right|^{p} \varphi d x+\int_{\Omega^{\prime}}\left|u_{n}-z_{n}\right|^{p} \varphi d \mu_{n}\right) \\
\leq C\left(\int_{\Omega^{\prime}}|u|^{p} \varphi d \mu+\int_{\Omega^{\prime}}|z|^{p} \varphi d \mu\right)^{\frac{p-2}{p-1}}\left(\int_{\Omega^{\prime}}|u-z|^{p} \varphi d \mu\right)^{\frac{1}{p-1}}
\end{gathered}
$$

where $C$ is a positive constant which depends only on $\alpha, \beta, L, N$, and $p$.

Proof. By Theorem 4.4 we have that $u$ and $z$ belong to $W^{1, p}\left(\Omega, \mathbf{R}^{M}\right) \cap L_{\mu}^{p}\left(\Omega, \mathbf{R}^{M}\right)$. Let $\varphi \in C_{c}^{\infty}\left(\Omega^{\prime}\right)$, with $\varphi \geq 0$, let $w_{n}$ and $w$ be the solutions of problems (3.5) and (3.3). By Proposition 3.6 there exists a sequence $\left(\psi_{m}\right)$ in $C_{0}^{\infty}\left(\Omega^{\prime}, \mathbf{R}^{M}\right)$ such that $\left(w \psi_{m}\right)$ converges to $u-z$ strongly in $W_{\text {loc }}^{1, p}\left(\Omega^{\prime}\right) \cap L_{\mu}^{p}\left(\Omega^{\prime}\right)$. Thus, taking $\left(u_{n}-z_{n}-w_{n} \psi_{m}\right) \varphi$ 
as a test function in the difference of the equations in (6.8), we get

$$
\begin{gathered}
\int_{\Omega^{\prime}}\left[a\left(x, D u_{n}\right)-a\left(x, D z_{n}\right)\right] D\left(u_{n}-z_{n}-w_{n} \psi_{m}\right) \varphi d x \\
+\int_{\Omega^{\prime}}\left[F_{n}\left(x, u_{n}\right)-F_{n}\left(x, z_{n}\right)\right]\left(u_{n}-z_{n}-w_{n} \psi_{m}\right) \varphi d \mu_{n} \\
=-\int_{\Omega^{\prime}}\left[a\left(x, D u_{n}\right)-a\left(x, D z_{n}\right)\right]\left(u_{n}-z_{n}-w_{n} \psi_{m}\right) \otimes D \varphi d x \\
\quad+\left\langle f_{n}-g_{n},\left(u_{n}-z_{n}-w_{n} \psi_{m}\right) \varphi\right\rangle=o_{m, n} .
\end{gathered}
$$

Let us estimate the terms which appear in (6.11). By using assumption (ii) and Proposition 5.4, the sequences $\left(\mid a\left(x, D u_{n}\right)-a\left(x,\left.D\left(u_{n}-u\right)\right|^{p^{\prime}}\right)\right.$ and $\left(\mid a\left(x, D z_{n}\right)-\right.$ $a\left(x,\left.D\left(z_{n}-z\right)\right|^{p^{\prime}}\right)$ are uniformly integrable and pointwise convergent respectively to $|a(x, D u)|^{p^{\prime}}$ and $|a(x, D z)|^{p^{\prime}}$. Therefore they converge strongly in $L^{1}\left(\Omega^{\prime}, \mathbf{M}^{M \times N}\right)$ and hence, from (6.11), we deduce

$$
\begin{gathered}
\int_{\Omega^{\prime}}\left[a\left(x, D\left(u_{n}-u\right)\right)-a\left(x, D\left(z_{n}-z\right)\right)\right] D\left(u_{n}-z_{n}-(u-z)\right) \varphi d x \\
+\int_{\Omega^{\prime}}\left[F_{n}\left(x, u_{n}\right)-F_{n}\left(x, z_{n}\right)\right]\left(u_{n}-z_{n}\right) \varphi d \mu_{n} \\
=o_{m, n}-\int_{\Omega^{\prime}}[a(x, D u)-a(x, D z)] D\left(u_{n}-z_{n}-w_{n} \psi_{m}\right) \varphi d x \\
+\int_{\Omega^{\prime}}\left[a\left(x, D\left(u_{n}-u\right)\right)-a\left(x, D\left(z_{n}-z\right)\right)\right] D\left(w_{n} \psi_{m}-(u-z)\right) \varphi d x \\
+\int_{\Omega^{\prime}}\left[F_{n}\left(x, u_{n}\right)-F_{n}\left(x, z_{n}\right)\right] w_{n} \psi_{m} \varphi d \mu_{n} .
\end{gathered}
$$

Since

$$
\int_{\Omega^{\prime}}[a(x, D u)-a(x, D z)] D\left(u_{n}-z_{n}-w_{n} \psi_{m}\right) \varphi d x=o_{m, n}
$$

by properties (i) and (ii) of $a$ and properties (II) and (III) of $F_{n}$, we get

$$
\begin{gathered}
\alpha \int_{\Omega^{\prime}}\left|D\left(u_{n}-z_{n}-(u-z)\right)\right|^{p} \varphi d x+\alpha \int_{\Omega^{\prime}}\left|u_{n}-z_{n}\right|^{p} \varphi d \mu_{n} \\
\leq \beta \int_{\Omega^{\prime}}\left(h+\left|D\left(u_{n}-u\right)\right|+\left|D\left(z_{n}-z\right)\right|\right)^{p-2}\left|D\left(u_{n}-z_{n}-(u-z)\right)\right|\left|D\left(w_{n} \psi_{m}-(u-z)\right)\right| \varphi d x \\
+L \int_{\Omega^{\prime}}\left(\left|u_{n}\right|+\left|z_{n}\right|\right)^{p-2}\left|u_{n}-z_{n}\right|\left|w_{n} \psi_{m}\right| \varphi d \mu_{n}+o_{m, n} \\
=\beta \int_{\Omega^{\prime}}\left(\left|D\left(u_{n}-u\right)\right|+\left|D\left(z_{n}-z\right)\right|\right)^{p-2}\left|D\left(u_{n}-z_{n}-(u-z)\right)\right|\left|D\left(w_{n} \psi_{m}-(u-z)\right)\right| \varphi d x \\
+L \int_{\Omega^{\prime}}\left(\left|u_{n}\right|+\left|z_{n}\right|\right)^{p-2}\left|u_{n}-z_{n}\right|\left|w_{n} \psi_{m}\right| \varphi d \mu_{n}+o_{m, n} .
\end{gathered}
$$


Using Young's inequality and then Hölder's inequality in (6.12), we obtain

$$
\begin{gathered}
\int_{\Omega^{\prime}}\left|D\left(\left(u_{n}-z_{n}\right)-(u-z)\right)\right|^{p} \varphi d x+\int_{\Omega^{\prime}}\left|u_{n}-z_{n}\right|^{p} \varphi d \mu_{n} \\
\leq C\left(\int_{\Omega^{\prime}}\left(\left|D\left(u_{n}-u\right)\right|+\left|D\left(z_{n}-z\right)\right|\right)^{p} \varphi d x\right)^{\frac{p-2}{p-1}}\left(\int_{\Omega^{\prime}}\left|D\left(w_{n} \psi_{m}-(u-z)\right)\right|^{p} \varphi d x\right)^{\frac{1}{p-1}} \\
+C\left(\int_{\Omega^{\prime}}\left(\left|u_{n}\right|+\left|z_{n}\right|\right)^{p} \varphi d \mu_{n}\right)^{\frac{p-2}{p-1}}\left(\int_{\Omega^{\prime}}\left|w_{n} \psi_{m}\right|^{p} \varphi d \mu_{n}\right)^{\frac{1}{p-1}}+o_{m, n} .
\end{gathered}
$$

Taking $z_{n}=z=0$ (and then $g_{n}=0$ ), in estimate (6.13), by Young's inequality, we get

$$
\begin{aligned}
& \int_{\Omega^{\prime}}\left|D\left(u_{n}-u\right)\right|^{p} \varphi d x+\int_{\Omega^{\prime}}\left|u_{n}\right|^{p} \varphi d \mu_{n} \\
& \quad \leq C \int_{\Omega^{\prime}}\left|D\left(w_{n} \psi_{m}-u\right)\right|^{p} \varphi d x+C \int_{\Omega^{\prime}}\left|w_{n} \psi_{m}\right|^{p} \varphi d \mu_{n}+o_{m, n},
\end{aligned}
$$

which by Lemma 4.3 implies (6.9).

Finally, in order to get (6.10), it is enough to apply, in estimate (6.13), estimate (6.9) for $u_{n}$ and $z_{n}$, and Lemma 4.3.

The following proposition gives a first version of the limit problem satisfied by $u$.

Proposition 6.7. Let us assume (6.5), (6.6), (6.7), and (6.8). Then there exists a w $\mu$-measurable vector function $H$, uniquely defined $\mu$-a.e. in $\Omega^{\prime}$, such that the function $u$ satisfies the problem

$$
\left\{\begin{array}{l}
u \in W^{1, p}\left(\Omega^{\prime}, \mathbf{R}^{M}\right) \cap L_{\mu}^{p}\left(\Omega^{\prime}, \mathbf{R}^{M}\right), \\
\int_{\Omega^{\prime}} a(x, D u) D v d x+\int_{\Omega^{\prime}} H v d \mu=\langle f, v\rangle \\
\forall v \in W_{0}^{1, p}\left(\Omega^{\prime}, \mathbf{R}^{M}\right) \cap L_{\mu}^{p}\left(\Omega^{\prime}, \mathbf{R}^{M}\right),
\end{array}\right.
$$

and

$$
|H| \leq C|u|^{p-1} \quad \text {-a.e. in } \Omega^{\prime} .
$$

Moreover, for every $\phi \in C_{c}^{\infty}\left(\Omega^{\prime}, \mathbf{R}^{M}\right)$ we have

$$
\begin{gathered}
\int_{\Omega^{\prime}} H w \phi d \mu \\
=\lim _{n \rightarrow \infty}\left[\int_{\Omega^{\prime}} a\left(x, D\left(u_{n}-u\right)\right) \phi \otimes D\left(w_{n}-w\right) d x+\int_{\Omega^{\prime}} F_{n}\left(x, u_{n}\right) w_{n} \phi d \mu_{n}\right],
\end{gathered}
$$

where $w_{n}$ and $w$ are the solutions of problems (3.5) and (3.3).

Proof. Given $\phi \in C_{c}^{\infty}\left(\Omega^{\prime}, \mathbf{R}^{M}\right)$, we take $w_{n} \phi$ as the test function in the equation satisfied by $u_{n}$ (see (6.8)) and we get

$$
\begin{gathered}
\int_{\Omega^{\prime}} a\left(x, D u_{n}\right) \phi \otimes D w_{n} d x+\int_{\Omega^{\prime}} a\left(x, D u_{n}\right) D \phi w_{n} d x \\
+\int_{\Omega^{\prime}} F_{n}\left(x, u_{n}\right) w_{n} \phi d \mu_{n}=\left\langle f_{n}, w_{n} \phi\right\rangle .
\end{gathered}
$$


By Remark 5.5 we have

$$
\lim _{n \rightarrow \infty}\left(\left\langle f_{n}, w_{n} \phi\right\rangle-\int_{\Omega^{\prime}} a\left(x, D u_{n}\right) D \phi w_{n} d x\right)=\langle f, w \phi\rangle-\int_{\Omega^{\prime}} a(x, D u) D \phi w d x .
$$

Let us define a distribution $T$ in $\Omega^{\prime}$ by

$$
\begin{aligned}
\langle T, \phi\rangle=\lim _{n \rightarrow \infty}[ & \int_{\Omega^{\prime}} a\left(x, D u_{n}\right) \phi \otimes D w_{n} d x \\
& \left.\quad-\int_{\Omega^{\prime}} a(x, D u) \phi \otimes D w d x+\int_{\Omega^{\prime}} F_{n}\left(x, u_{n}\right) w_{n} \phi d \mu_{n}\right]
\end{aligned}
$$

for every $\phi \in C_{c}^{\infty}\left(\Omega^{\prime}, \mathbf{R}^{M}\right)$. Since the norm of $w_{n}$ in $W_{0}^{1, p}(\Omega) \cap L_{\mu_{n}}^{p}(\Omega)$ is bounded, by (6.1) and property $(\mathrm{V})$ we have

$$
\int_{\Omega}\left|a\left(x, D u_{n}\right)\right|\left|D w_{n}\right| d x+\int_{\Omega}\left|F_{n}\left(x, u_{n}\right)\right|\left|w_{n}\right| d \mu_{n} \leq C,
$$

and hence $T$ is continuous with respect to the uniform convergence and it can be represented by a vector Radon measure $\left(T_{1}, \ldots, T_{M}\right)$ such that

$$
\langle T, \phi\rangle=\sum_{i=1}^{M} \int_{\Omega^{\prime}} \phi_{i} d T_{i} \quad \forall \phi \in C_{c}^{\infty}\left(\Omega^{\prime}, \mathbf{R}^{M}\right),
$$

where $\phi_{1}, \ldots, \phi_{M}$ are the components of the vector function $\phi$. Thus taking the limit in (6.17) we obtain

$$
\int_{\Omega^{\prime}} a(x, D u) D(w \phi) d x+\langle T, \phi\rangle=\langle f, w \phi\rangle .
$$

Since by conditions (ii) and (iv) and Proposition 5.4, $a\left(x, D u_{n}\right)-a\left(x, D\left(u_{n}-u\right)\right)$ converges to $a(x, D u)$ strongly in $L^{p^{\prime}}\left(\Omega, \mathbf{M}^{M \times N}\right)$, we can write

$$
\langle T, \phi\rangle=\lim _{n \rightarrow \infty}\left[\int_{\Omega^{\prime}} a\left(x, D\left(u_{n}-u\right)\right) \phi \otimes D\left(w_{n}-w\right) d x+\int_{\Omega^{\prime}} F_{n}\left(x, u_{n}\right) w_{n} \phi d \mu_{n}\right] .
$$

Let us prove (6.16). For every $\phi \in C_{c}^{\infty}\left(\Omega^{\prime}, \mathbf{R}^{M}\right)$, with $\phi \geq 0$, by assumptions (V) and (v), Proposition 5.4, Hölder's inequality, Lemma 4.3, and estimate (6.9), we have

$$
\begin{aligned}
|\langle T, \phi\rangle| \leq C \limsup _{n \rightarrow \infty}\left[\int_{\Omega^{\prime}}\left(k(x)+\eta\left|D\left(u_{n}-u\right)\right|^{p-1}\right)\left|D\left(w_{n}-w\right)\right||\phi| d x\right. \\
\left.\quad+\int_{\Omega^{\prime}}\left|F_{n}\left(x, u_{n}\right)\right| w_{n}|\phi| d \mu_{n}\right] \\
\leq C \limsup _{n \rightarrow \infty}\left[\int_{\Omega}\left|D\left(u_{n}-u\right)\right|^{p-1}\left|D\left(w_{n}-w\right)\right||\phi| d x+\int_{\Omega^{\prime}}\left|u_{n}\right|^{p-1} w_{n}|\phi| d \mu_{n}\right] \\
(6.21) \leq C \limsup _{n \rightarrow \infty}\left[\left(\int_{\Omega^{\prime}}\left|D\left(u_{n}-u\right)\right|^{p}|\phi| d x\right)^{\frac{p-1}{p}}\left(\int_{\Omega}\left|D\left(w_{n}-w\right)\right|^{p}|\phi| d x\right)^{\frac{1}{p}}\right. \\
\left.\quad+\left(\int_{\Omega^{\prime}}\left|u_{n}\right|^{p}|\phi| d \mu_{n}\right)^{\frac{p-1}{p}}\left(\int_{\Omega^{\prime}}\left|w_{n}\right|^{p}|\phi| d \mu_{n}\right)^{\frac{1}{p}}\right] \\
\leq C\left(\int_{\Omega^{\prime}}|u|^{p}|\phi| d \mu\right)^{\frac{p-1}{p}}\left(\int_{\Omega^{\prime}}|w|^{p}|\phi| d \mu\right)^{\frac{1}{p}} .
\end{aligned}
$$


Let us denote by $\left|T_{i}\right|$ the total variation of the measures $T_{i}, i=1 \ldots, M$. Taking into account that for every open subset $A$ of $\Omega^{\prime}$ we have

$$
\left|T_{i}\right|(A)=\sup \left\{\left\langle T_{i}, \varphi\right\rangle: \varphi \in C_{0}^{\infty}(A), \sup |\varphi| \leq 1\right\},
$$

by $(6.21)$ we get

$$
\left|T_{i}\right|(A) \leq C\left(\int_{A}|u|^{p} d \mu\right)^{\frac{p-1}{p}}\left(\int_{A}|w|^{p} d \mu\right)^{\frac{1}{p}}
$$

for every open subset $A$ of $\Omega^{\prime}$. Since $|u|^{p} \mu,|w|^{p} \mu$, and $\left|T_{i}\right|$ are finite measures, (6.22) holds for every Borel subset of $\Omega^{\prime}$. This implies that the measures $T_{i}$ are absolutely continuous with respect to the measure $|w|^{p} \mu$, and hence to the measure $w \mu$. Since $w \mu$ is a $\sigma$-finite measure we can apply the Radon-Nikodým derivation theorem and we find a $w \mu$-measurable vector function $H=\left(H_{1}, \ldots, H_{M}\right)$ such that

$$
T_{i}(A)=\int_{A} H_{i} w d \mu
$$

for every Borel subset $A$ of $\Omega^{\prime}$ and $i=1, \ldots, M$, so that, by (6.20) and (6.18), (6.16) holds. We can suppose that

$$
H_{i}(x)=0 \quad \text { for } \mu \text {-a.e. } x \text { in }\{w=0\} \quad \forall i=1, \ldots, M .
$$

Thus by (6.22) we get

$$
\int_{A}\left|H_{i}\right| w d \mu \leq C\left(\int_{A}|u|^{p} d \mu\right)^{\frac{p-1}{p}}\left(\int_{A}|w|^{p} d \mu\right)^{\frac{1}{p}}
$$

for every Borel subset $A$ of $\Omega^{\prime}$. Using Young's inequality, we obtain

$$
\int_{A}\left|H_{i}\right| w d \mu \leq C\left(\frac{1}{p^{\prime} \varepsilon^{p^{\prime}}} \int_{A}|u|^{p} d \mu+\frac{\varepsilon^{p}}{p} \int_{A}|w|^{p} d \mu\right)
$$

for every Borel subset $A$ of $\Omega^{\prime}$ and for every $\varepsilon>0$. Thus (first reasoning for $\varepsilon \in \mathbf{Q}$ and then arguing by density) we get

$$
\left|H_{i}(x)\right| w(x) \leq C\left(\frac{1}{p^{\prime} \varepsilon^{p^{\prime}}}|u(x)|^{p}+\frac{\varepsilon^{p}}{p}|w(x)|^{p}\right)
$$

for $\mu$-a.e. $x$ in $\Omega^{\prime}$ and for every $\varepsilon>0$. If $x \in \Omega^{\prime}$ satisfies $w(x)>0$ and (6.24) holds true for any $\varepsilon$, by choosing $\varepsilon=|u(x)|^{\frac{p-1}{p}} /|w(x)|^{\frac{p-1}{p}}$ in (6.24) and taking into account (6.23), we get

$$
\left|H_{i}(x)\right| \leq C|u(x)|^{p-1}, \quad \mu \text {-a.e. } x \in \Omega^{\prime},
$$

and hence (6.15) is proved. Condition (6.14) follows from (6.19), (6.18), and the density result given by Proposition 3.6. Finally the vector function $H$ is uniquely determined $\mu$-a.e. in $\Omega^{\prime}$ by (6.14) and (6.15). Indeed, by (6.14) $H$ is uniquely determined $\mu$-a.e. in $\{w>0\}$, and by $(6.15)$ we have $H=0 \mu$-a.e. in $\{|u|=0\}_{\Omega^{\prime}}$. Then the conclusion follows by Lemma 4.5.

In order to study the dependence of the function $H$ on the function $u$, let us consider a sequence of functionals $\left(g_{n}\right)$ and a sequence of functions $\left(z_{n}\right)$ which satisfy 
(6.6), (6.7), and (6.8). By Proposition 6.7, applied to $\left(z_{n}\right)$, we get that there exists a $w \mu$-measurable vector function $H^{\prime}$, uniquely defined $\mu$-a.e. in $\Omega^{\prime}$, such that

$$
\begin{aligned}
& \left\{\begin{array}{l}
z \in W^{1, p}\left(\Omega^{\prime}, \mathbf{R}^{M}\right) \cap L_{\mu}^{p}\left(\Omega^{\prime}, \mathbf{R}^{M}\right), \\
\int_{\Omega^{\prime}} a(x, D z) D v d x+\int_{\Omega^{\prime}} H^{\prime} v d \mu=\langle g, v\rangle \\
\forall v \in W_{0}^{1, p}\left(\Omega^{\prime}, \mathbf{R}^{M}\right) \cap L_{\mu}^{p}\left(\Omega^{\prime}, \mathbf{R}^{M}\right),
\end{array}\right. \\
& \left|H^{\prime}\right| \leq C|z|^{p-1}, \quad \mu \text {-a.e. in } \Omega^{\prime},
\end{aligned}
$$

and

$$
\begin{gathered}
\int_{\Omega^{\prime}} H^{\prime} w \phi d \mu \\
=\lim _{n \rightarrow \infty}\left[\int_{\Omega^{\prime}} a\left(x, D\left(z_{n}-z\right)\right) \phi \otimes D\left(w_{n}-w\right) d x+\int_{\Omega^{\prime}} F_{n}\left(x, z_{n}\right) w_{n} \phi d \mu_{n}\right] .
\end{gathered}
$$

The following proposition compares the function $H$ with the function $H^{\prime}$.

Proposition 6.8. The vector functions $H$ and $H^{\prime}$ satisfy

$$
\left|H-H^{\prime}\right| \leq C(|u|+|z|)^{\frac{p-2}{p-1}}|u-z|^{\frac{1}{p-1}}, \quad \mu \text {-a.e. in } \Omega^{\prime}
$$

and

$$
\left(H-H^{\prime}\right)(u-z) \geq \alpha|u-z|^{p}, \quad \mu \text {-a.e. in } \Omega^{\prime} .
$$

Proof. Let us first prove (6.28). Consider $\phi \in C_{c}^{\infty}\left(\Omega^{\prime}, \mathbf{R}^{M}\right)$ and let $w_{n}$ and $w$ be the solutions of problems (3.5) and (3.3). By (6.16), (6.27), and by assumptions (ii) and (III), we have

$$
\begin{gathered}
\left|\int_{\Omega^{\prime}}\left(H-H^{\prime}\right) w \phi d \mu\right| \\
\leq\left|\int_{\Omega^{\prime}}\left(a\left(x, D\left(u_{n}-u\right)\right)-a\left(x, D\left(z_{n}-z\right)\right)\right) \phi \otimes D\left(w_{n}-w\right) d x\right| \\
+\left|\int_{\Omega^{\prime}}\left(F_{n}\left(x, u_{n}\right)-F_{n}\left(x, z_{n}\right)\right) w_{n} \phi d \mu_{n}\right|+o_{n} \\
\leq C \int_{\Omega^{\prime}}\left(h+\left|D\left(u_{n}-u\right)\right|+\left|D\left(z_{n}-z\right)\right|\right)^{p-2}\left|D\left(\left(u_{n}-z_{n}\right)-(u-z)\right)\right|\left|D\left(w_{n}-w\right)\right||\phi| d x \\
+C \int_{\Omega^{\prime}}\left(\left|u_{n}\right|+\left|z_{n}\right|\right)^{p-2}\left|u_{n}-z_{n}\right||\phi| w_{n} d \mu_{n}+o_{n} \\
\leq C \int_{\Omega^{\prime}}\left(\left|D\left(u_{n}-u\right)\right|+\left|D\left(z_{n}-z\right)\right|\right)^{p-2}\left|D\left(\left(u_{n}-z_{n}\right)-(u-z)\right)\right|\left|D\left(w_{n}-w\right)\right||\phi| d x \\
+C \int_{\Omega^{\prime}}\left(\left|u_{n}\right|+\left|z_{n}\right|\right)^{p-2}\left|u_{n}-z_{n}\right||\phi| w_{n} d \mu_{n}+o_{n} .
\end{gathered}
$$

By using Hölder's inequality, (6.9), applied to $u_{n}$ and $z_{n}$ and (6.10), we get

$$
\begin{gathered}
\left|\int_{\Omega^{\prime}}\left(H-H^{\prime}\right) w \phi d \mu\right| \\
\leq C\left(\int_{\Omega^{\prime}}|u|^{p}|\phi| d \mu+\int_{\Omega}|z|^{p}|\phi| d \mu\right)^{\frac{p-2}{p-1}}\left(\int_{\Omega^{\prime}}|u-z|^{p}|\phi| d \mu\right)^{\frac{1}{p(p-1)}}\left(\int_{\Omega^{\prime}}|w|^{p}|\phi| d \mu\right)^{\frac{1}{p}} .
\end{gathered}
$$


Then we conclude as in the proof of Proposition 6.7 and we obtain (6.28).

In order to prove (6.29), let us consider a function $\varphi \in C_{c}^{\infty}\left(\Omega^{\prime}\right)$, with $\varphi \geq 0$. Using $\left(u_{n}-z_{n}\right) \varphi$ as a test function in the difference of the two equations in (6.8), we obtain

$$
\begin{gathered}
\int_{\Omega^{\prime}}\left[a\left(x, D u_{n}\right)-a\left(x, D z_{n}\right)\right] D\left(u_{n}-z_{n}\right) \varphi d x \\
+\int_{\Omega^{\prime}}\left[a\left(x, D u_{n}\right)-a\left(x, D z_{n}\right)\right]\left(u_{n}-z_{n}\right) \otimes D \varphi d x \\
+\int_{\Omega^{\prime}}\left[F_{n}\left(x, u_{n}\right)-F_{n}\left(x, z_{n}\right)\right]\left(u_{n}-z_{n}\right) \varphi d \mu_{n}=\left\langle f_{n}-g_{n},\left(u_{n}-z_{n}\right) \varphi\right\rangle .
\end{gathered}
$$

We can rewrite this formula as

$$
\begin{gathered}
\int_{\Omega^{\prime}}\left(\left[a\left(x, D u_{n}\right)-a\left(x, D z_{n}\right)\right] D\left(u_{n}-z_{n}\right)-\alpha\left|D\left(u_{n}-z_{n}\right)\right|^{p}\right) \varphi d x \\
(6.31)+\alpha \int_{\Omega^{\prime}}\left|D\left(u_{n}-z_{n}\right)\right|^{p} \varphi d x+\int_{\Omega^{\prime}}\left[F_{n}\left(x, u_{n}\right)-F_{n}\left(x, z_{n}\right)\right]\left(u_{n}-z_{n}\right) \varphi d \mu_{n} \\
+\int_{\Omega^{\prime}}\left[a\left(x, D u_{n}\right)-a\left(x, D z_{n}\right)\right]\left(u_{n}-z_{n}\right) \otimes D \varphi d x=\left\langle f_{n}-g_{n},\left(u_{n}-z_{n}\right) \varphi\right\rangle .
\end{gathered}
$$

By assumption (II) and Theorem 4.4, we have

$$
\begin{gathered}
\alpha \int_{\Omega^{\prime}}\left|D\left(u_{n}-z_{n}\right)\right|^{p} \varphi d x+\int_{\Omega^{\prime}}\left[F_{n}\left(x, u_{n}\right)-F_{n}\left(x, z_{n}\right)\right]\left(u_{n}-z_{n}\right) \varphi d \mu_{n} \\
\geq \alpha \int_{\Omega^{\prime}}|D(u-z)|^{p} \varphi d x+\alpha \int_{\Omega^{\prime}}|u-z|^{p} \varphi d \mu+o_{n} .
\end{gathered}
$$

Moreover, by Remark 5.5 , the sequence $\left(a\left(x, D u_{n}\right)-a\left(x, D z_{n}\right)\right)$ converges to $a(x, D u)-$ $a(x, D z)$ pointwise a.e. in $\Omega^{\prime}$ and weakly in $L^{p^{\prime}}\left(\Omega^{\prime}, \mathbf{M}^{M \times N}\right)$. Then by condition (i) we can apply Fatou's lemma to the first integrand of (6.31) and, taking the limit, we obtain

$$
\begin{gathered}
\int_{\Omega^{\prime}}\left([a(x, D u)-a(x, D z)] D(u-z)-\alpha|D(u-z)|^{p}\right) \varphi d x \\
+\alpha \int_{\Omega^{\prime}}|D(u-z)|^{p} \varphi d x+\alpha \int_{\Omega^{\prime}}|u-z|^{p} \varphi d \mu \\
+\int_{\Omega^{\prime}}[a(x, D u)-a(x, D z)](u-z) \otimes D \varphi d x \leq\langle f-g,(u-z) \varphi\rangle,
\end{gathered}
$$

that is,

$$
\int_{\Omega^{\prime}}[a(x, D u)-a(x, D z)] D(\varphi(u-z)) d x+\alpha \int_{\Omega^{\prime}}|u-z|^{p} \varphi d \mu \leq\langle f-g,(u-z) \varphi\rangle .
$$

Thus by (6.14) and (6.25) we get

$$
\int_{\Omega^{\prime}}\left(H-H^{\prime}\right)(u-z) \varphi d \mu \geq \alpha \int_{\Omega^{\prime}}|u-z|^{p} \varphi d \mu
$$

for every $\varphi \in C_{c}^{\infty}\left(\Omega^{\prime}\right)$, with $\varphi \geq 0$. This implies (6.29).

Proposition 6.8 will imply that the function $H$ defined by (6.16) depends on $u$ only through its pointwise values, i.e., there exists a function $F(x, s)$ such that 
$H(x)=F(x, u(x)) \mu$-a.e. in $\Omega$. This construction allows us to define the function $F(x, s)$ only on the pairs $(x, s)$ such that $s=u(x)$, where $u$ is the limit of a sequence of solutions of problems (5.2). We shall prove a penalization result (Theorem 6.9) which shows that, in some sense, it is possible to obtain any real number $s$ as the "limit" of a sequence of solutions.

Theorem 6.9. Let $s \in \mathbf{R}^{M}$. For every $m \in \mathbf{N}$, let $s_{n}^{m}$ be the unique solution of the problem

$$
\left\{\begin{array}{l}
s_{n}^{m} \in W_{0}^{1, p}\left(\Omega, \mathbf{R}^{M}\right) \cap L_{\mu_{n}}^{p}\left(\Omega, \mathbf{R}^{M}\right), \\
\int_{\Omega} a\left(x, D s_{n}^{m}\right) D v d x+\int_{\Omega} F_{n}\left(x, s_{n}^{m}\right) v d \mu_{n} \\
=m \int_{\Omega}\left(\left|w_{n} s\right|^{p-2} w_{n} s-\left|s_{n}^{m}\right|^{p-2} s_{n}^{m}\right) v d x \\
\forall v \in W_{0}^{1, p}\left(\Omega, \mathbf{R}^{M}\right) \cap L_{\mu_{n}}^{p}\left(\Omega, \mathbf{R}^{M}\right) .
\end{array}\right.
$$

Then there exists an increasing sequence of indices $\left(n_{j}\right)$ such that for every $m$ the sequence $\left(s_{n_{j}}^{m}\right)_{j \in \mathbf{N}}$ converges to some function $s^{m}$ weakly in $W_{0}^{1, p}\left(\Omega, \mathbf{R}^{M}\right)$. The sequence $\left(s^{m}\right)$ converges to ws strongly in $W_{0}^{1, p}\left(\Omega, \mathbf{R}^{M}\right) \cap L_{\mu}^{p}\left(\Omega, \mathbf{R}^{M}\right)$ and satisfies

$$
\lim _{m \rightarrow \infty} m \int_{\Omega}\left|s^{m}-w s\right|^{p} d x=0
$$

Moreover, there exists a unique wh-measurable function $H_{s}^{m}$, with

$$
\left|H_{s}^{m}\right| \leq C\left|s^{m}\right|^{p-1}, \quad \mu \text {-a.e. in } \Omega \text {, }
$$

such that the function $s^{m}$ satisfies the problem

$$
\left\{\begin{array}{l}
s^{m} \in W_{0}^{1, p}\left(\Omega, \mathbf{R}^{M}\right) \cap L_{\mu}^{p}\left(\Omega, \mathbf{R}^{M}\right), \\
\int_{\Omega} a\left(x, D s^{m}\right) D v d x+\int_{\Omega} H_{s}^{m} v d \mu \\
=m \int_{\Omega}\left(\left|w_{n} \psi\right|^{p-2} w_{n} \psi-\left|s^{m}\right|^{p-2} s^{m}\right) v d x \\
\forall v \in W_{0}^{1, p}\left(\Omega, \mathbf{R}^{M}\right) \cap L_{\mu}^{p}\left(\Omega, \mathbf{R}^{M}\right) .
\end{array}\right.
$$

The sequence $\left(H_{s}^{m}\right)$ converges in $L_{\mu}^{p^{\prime}}\left(\Omega, \mathbf{R}^{M}\right)$ to a function $H_{s}$ which satisfies

$$
\left|H_{s}\right| \leq C\left|s^{m}\right|^{p-1}, \quad \mu \text {-a.e. in } \Omega \text {. }
$$

Proof. Let $s \in \mathbf{R}^{M}$ and let $s_{n}^{m} \in W_{0}^{1, p}\left(\Omega, \mathbf{R}^{M}\right) \cap L_{\mu_{n}}^{p}\left(\Omega, \mathbf{R}^{M}\right)$ be the solution of 
problem (6.32). Taking $\left(s_{n}^{m}-w_{n} s\right)$ as the test function in (6.32) we get

$$
\begin{gathered}
\int_{\Omega}\left(a\left(x, D s_{n}^{m}\right)-a\left(x, D\left(w_{n} s\right)\right)\right) D\left(s_{n}^{m}-w_{n} s\right) d x \\
+\int_{\Omega}\left(F\left(x, s_{n}^{m}\right)-F\left(x, w_{n} s\right)\right)\left(s_{n}^{m}-w_{n} s\right) d \mu_{n} \\
+m \int_{\Omega}\left(\left|s_{n}^{m}\right|^{p-2} s_{n}^{m}-\left|w_{n} s\right|^{p-2} w_{n} s\right)\left(s_{n}^{m}-w_{n} s\right) d x \\
=-\int_{\Omega} a\left(x, D\left(w_{n} s\right)\right) D\left(s_{n}^{m}-w_{n} s\right) d x-\int_{\Omega} F\left(x, w_{n} s\right)\left(s_{n}^{m}-w_{n} s\right) d \mu_{n} .
\end{gathered}
$$

Using assumptions (i) and (iv) of $a$, (II) and (V) of $F_{n}$, and (4.3) we obtain

$$
\begin{aligned}
& \alpha \int_{\Omega}\left|D\left(s_{n}^{m}-w_{n} s\right)\right|^{p} d x+\alpha \int_{\Omega}\left|s_{n}^{m}-w_{n} s\right|^{p} d \mu_{n}+m 2^{2-p} \int_{\Omega}\left|s_{n}^{m}-w_{n} s\right|^{p} d x \\
\leq & \int_{\Omega}\left(k(x)+\eta\left|D\left(w_{n} s\right)\right|^{p-1}\right)\left|D\left(s_{n}^{m}-w_{n} s\right)\right| d x+L \int_{\Omega}\left|w_{n} s\right|^{p-1}\left|s_{n}^{m}-w_{n} s\right| d \mu_{n} .
\end{aligned}
$$

Then, by Young's inequality and the fact that $\int_{\Omega}\left|D w_{n}\right|^{p} d x+\int_{\Omega}\left|w_{n}\right|^{p} d \mu_{n}$ is bounded, it is easy to see that there exists a constant $C$ such that

$$
\int_{\Omega}\left|D\left(s_{n}^{m}-w_{n} s\right)\right|^{p} d x+\int_{\Omega}\left|s_{n}^{m}-w_{n} s\right|^{p} d \mu_{n}+m \int_{\Omega}\left|s_{n}^{m}-w_{n} s\right|^{p} d x \leq C|s|^{p} .
$$

Then there exists an increasing sequence of indices $\left(n_{j}\right)$ which, by a diagonal procedure, we can assume independent on $m$, such that for every $m \in \mathbf{N}$ the sequence $\left(s_{n_{j}}^{m}\right)_{j \in \mathbf{N}}$ converges to some function $s^{m}$ weakly in $W_{0}^{1, p}\left(\Omega, \mathbf{R}^{M}\right)$. Moreover, by Theorem 4.4 we have

$$
\int_{\Omega}\left|D\left(s^{m}-w s\right)\right|^{p} d x+\int_{\Omega}\left|s^{m}-w s\right|^{p} d \mu+m \int_{\Omega}\left|s^{m}-w s\right|^{p} d x \leq C|s|^{p} .
$$

This implies that $\left(s^{m}\right)$ converges weakly in $W_{0}^{1, p}\left(\Omega, \mathbf{R}^{M}\right)$ to $w s$. In particular $\left|s^{m}-w s\right|$ converges to zero weakly in $W_{0}^{1, p}(\Omega)$, and by Theorem 3.5 we get

$$
\lim _{m \rightarrow \infty} \int_{\Omega}\left|s^{m}-w s\right| w^{p-1} d \mu=\lim _{m \rightarrow \infty} \int_{\Omega}\left|s^{m}-w s\right| d \nu=0 .
$$

Thus up to a subsequence $\left(s^{m}\right)$ converges to $w s \nu$-a.e. in $\Omega$ and hence by Lemma 4.5 $\mu$-a.e. in $\Omega$. Moreover, since by $(6.39)\left(s^{m}\right)$ is bounded in $L_{\mu}^{p}\left(\Omega, \mathbf{R}^{M}\right)$, it converges to ws weakly in $L_{\mu}^{p}\left(\Omega, \mathbf{R}^{M}\right)$.

By Proposition 6.7, for every $m \in \mathbf{N}$, there exists a $w \mu$-measurable vector function $H_{s}^{m}$, uniquely defined $\mu$-a.e. in $\Omega$, which satisfies (6.33) and such that $s^{m}$ is the solution of the problem (6.34). By Proposition 6.8, for every $m, k \in \mathbf{N}$, we have

$$
\left|H_{s}^{m}-H_{s}^{k}\right| \leq C\left(\left|s^{m}\right|+\left|s^{k}\right|\right)^{\frac{p-2}{p-1}}\left|s^{m}-s^{k}\right|^{\frac{1}{p-1}}, \quad \mu \text {-a.e. in } \Omega .
$$

This implies that there exists a function $H_{s}$, which satisfies (6.35), such that $H_{s}^{m}$ converges to $H_{s} \mu$-a.e. in $\Omega$. Moreover, by Proposition 6.8 , for every $m, k \in \mathbf{N}$, we have

$$
\left(H_{s}^{m}-H_{s}^{k}\right)\left(s^{m}-s^{k}\right) \geq \alpha\left|s^{m}-s^{k}\right|^{p}, \quad \mu \text {-a.e. in } \Omega,
$$


and then, taking the limit as $k \rightarrow \infty$, we obtain

$$
\left(H_{s}^{m}-H_{s}\right)\left(s^{m}-w s\right) \geq \alpha\left|s^{m}-w s\right|^{p}, \quad \mu \text {-a.e. in } \Omega .
$$

Now, taking $\left(s^{m}-w s\right)$ as a test function in $(6.34)$, we get

$$
\begin{aligned}
\int_{\Omega}\left(a\left(x, D s^{m}\right)\right. & -a(x, D(w s))) D\left(s^{m}-w s\right) d x+\int_{\Omega}\left(H_{s}^{m}-H_{s}\right)\left(s^{m}-w s\right) d \mu \\
& +m \int_{\Omega}\left(\left|s^{m}\right|^{p-2} s^{m}-|w s|^{p-2} w s\right)\left(s^{m}-w s\right) d x \\
=- & \int_{\Omega} a(x, D(w s)) D\left(s^{m}-w s\right) d x-\int_{\Omega} H_{s}\left(s^{m}-w s\right) d \mu .
\end{aligned}
$$

Then by (6.41), assumption (i), and the inequality (4.3), we obtain

$$
\begin{gathered}
\alpha \int_{\Omega}\left|D\left(s^{m}-w s\right)\right|^{p} d x+\alpha \int_{\Omega}\left|s^{m}-w s\right|^{p} d \mu \\
\leq-\int_{\Omega} a(x, D(w s)) D\left(s^{m}-w s\right) d x-\int_{\Omega} H_{s}\left(s^{m}-w s\right) d \mu .
\end{gathered}
$$

The conclusion follows by the weak convergence of $\left(s^{m}\right)$ to $w s$ in $W_{0}^{1, p}\left(\Omega, \mathbf{R}^{M}\right) \cap$ $L_{\mu}^{p}\left(\Omega, \mathbf{R}^{M}\right)$.

We are now in a position to prove Theorem 6.4.

Proof of Theorem 6.4. We start by defining the sequence $\left(n_{j}\right)$, the measure $\mu$, and the function $F$. By Theorem 3.4 we can suppose that there exists a measure

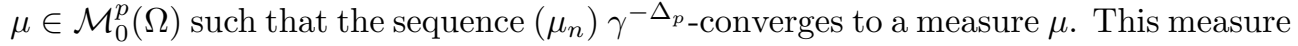
will be the measure which appears in the statement.

For any $q \in \mathbf{Q}^{M}$, let $q_{n}^{m}$ be the solutions of the problems

$$
\left\{\begin{array}{l}
q_{n}^{m} \in W_{0}^{1, p}\left(\Omega, \mathbf{R}^{M}\right) \cap L_{\mu}^{p}\left(\Omega, \mathbf{R}^{M}\right) \\
\int_{\Omega} a\left(x, D q_{n}^{m}\right) D v d x+\int_{\Omega} F_{n}\left(x, q_{n}^{m}\right) v d \mu_{n} \\
\quad=m \int_{\Omega}\left(\left|w_{n} q\right|^{p-2} w_{n} q-\left|q_{n}^{m}\right|^{p-2} q_{n}^{m}\right) v d x \\
\forall v \in W_{0}^{1, p}\left(\Omega, \mathbf{R}^{M}\right) \cap L_{\mu}^{p}\left(\Omega, \mathbf{R}^{M}\right) .
\end{array}\right.
$$

By Theorem 6.9 and a diagonal argument, there exists an increasing sequence $\left(n_{j}\right)$ such that for every $q \in \mathbf{Q}^{M}$, the sequence $\left(q_{n_{j}}^{m}\right)$ converges weakly in $W_{0}^{1, p}\left(\Omega, \mathbf{R}^{M}\right)$ to a function $q^{m} \in W_{0}^{1, p}\left(\Omega, \mathbf{R}^{M}\right) \cap L_{\mu}^{p}\left(\Omega, \mathbf{R}^{M}\right)$ when $j$ tends to infinity, and the sequence $\left(q^{m}\right)$ converges strongly in $W_{0}^{1, p}\left(\Omega, \mathbf{R}^{M}\right) \cap L_{\mu}^{p}\left(\Omega, \mathbf{R}^{M}\right)$ to $q w$ when $m$ tends to infinity. Moreover, there exists a sequence $\left(H_{q}^{m}\right)$ in $L_{\mu}^{p^{\prime}}\left(\Omega, \mathbf{R}^{M}\right)$ such that the sequence $\left(q^{m}\right)$ 
satisfies the problem

$$
\left\{\begin{array}{l}
q^{m} \in W_{0}^{1, p}\left(\Omega, \mathbf{R}^{M}\right) \cap L_{\mu}^{p}\left(\Omega, \mathbf{R}^{M}\right) \\
\int_{\Omega} a\left(x, D q^{m}\right) D v d x+\int_{\Omega} H_{q}^{m} v d \mu \\
\quad=m \int_{\Omega}\left(\left|w_{n} q\right|^{p-2} w_{n} q-\left|q^{m}\right|^{p-2} q^{m}\right) v d x \\
\forall v \in W_{0}^{1, p}\left(\Omega, \mathbf{R}^{M}\right) \cap L_{\mu}^{p}\left(\Omega, \mathbf{R}^{M}\right)
\end{array}\right.
$$

and such that it converges strongly in $L_{\mu}^{p^{\prime}}\left(\Omega, \mathbf{R}^{M}\right)$ to a function $H_{q}$ which satisfies

$$
\left|H_{q}\right| \leq C|w q|^{p-1}, \quad \mu \text {-a.e. in } \Omega .
$$

Applying Proposition 6.8 to $q_{n}^{m}$ and $\left(q^{\prime}\right)_{n}^{m}$ and then taking the limit in $H_{q}^{m}$ and $H_{q^{\prime}}^{m}$, we also have

$$
\begin{aligned}
& \left|H_{q}(x)-H_{q^{\prime}}(x)\right| \leq C\left(|q|+\left|q^{\prime}\right|\right)^{p \frac{p-2}{p-1}}\left|q-q^{\prime}\right|^{\frac{1}{p-1}} w(x)^{p-1} \\
& \quad \forall q, q^{\prime} \in \mathbf{Q}^{M}, \mu \text {-a.e. } x \text { in } \Omega
\end{aligned}
$$

and

$$
\left(H_{q}(x)-H_{q^{\prime}}(x)\right)\left(q-q^{\prime}\right) \geq \alpha\left|q-q^{\prime}\right|^{p} w(x)^{p} \quad \forall q, q^{\prime} \in \mathbf{Q}^{M}, \mu \text {-a.e. } x \text { in } \Omega .
$$

We define a function $G: \Omega \times \mathbf{Q}^{M} \mapsto \mathbf{R}^{M}$ by

$$
G(x, q)=H_{q}(x) \quad \forall q \in \mathbf{Q}^{M} \text {, } \mu \text {-a.e. } x \text { in } \Omega
$$

and then we extend $G$ to $\Omega \times \mathbf{R}^{M}$ by continuity (see (6.45)). The function $G$ satisfies

$$
\left\{\begin{array}{l}
|G(x, s)| \leq C|s|^{p-1} w(x)^{p-1} \\
\left|G(x, s)-G\left(x, s^{\prime}\right)\right| \leq C\left(|s|+\left|s^{\prime}\right|\right)^{p \frac{p-2}{p-1}}\left|s_{1}-s_{2}\right|^{\frac{1}{p-1}} w(x)^{p-1} \\
\left(G(x, s)-G\left(x, s^{\prime}\right)\right)\left(s-s^{\prime}\right) \geq \alpha\left|s-s^{\prime}\right|^{p} w(x)^{p^{p}}
\end{array}\right.
$$

for every $s$ and $s^{\prime}$ in $\mathbf{R}^{M}$ and for $\mu$-almost every $x$ in $\Omega$, and it is a Carathéodory function with respect to the $\sigma$-finite measure $w \mu$. Therefore, there exists a Borel function $F: \Omega \times \mathbf{R}^{M} \rightarrow \mathbf{R}^{M}$ such that

$$
F(x, s)=G\left(x, \frac{s}{w(x)}\right) 1_{\{w>0\}}(x)+\alpha|s|^{p-2} s 1_{\{w=0\}} \quad \forall s \in \mathbf{R}^{M}, \mu \text {-a.e. } x \text { in } \Omega,
$$

so that, by (6.48), $F \in \mathcal{F}(\alpha, C, 1 /(p-1))$.

In order to prove Theorem 6.4 it remains only to show that the pairs $\left(\mu_{n_{j}}, F_{n_{j}}\right)$ $\gamma^{A}$-converge to $(\mu, F)$. To carry this out, consider an open subset $\Omega^{\prime}$ of $\Omega$ and a sequence of functionals $\left(f_{n_{j}}\right)$, with $f_{n_{j}} \in\left(W_{0}^{1, p}\left(\Omega^{\prime}, \mathbf{R}^{M}\right) \cap L_{\mu_{n_{j}}}^{p}\left(\Omega^{\prime}, \mathbf{R}^{M}\right)\right)^{\prime}$, which converges in the sense of $\left(\mathcal{H}_{\Omega^{\prime}}\right)$ to a functional $f \in\left(W_{0}^{1, p}\left(\Omega^{\prime}, \mathbf{R}^{M}\right) \cap L_{\mu}^{p}\left(\Omega^{\prime}, \mathbf{R}^{M}\right)\right)^{\prime}$. We have to prove that if $u_{n_{j}} \in W_{0}^{1, p}\left(\Omega^{\prime}, \mathbf{R}^{M}\right) \cap L_{\mu_{n_{j}}}^{p}\left(\Omega^{\prime}, \mathbf{R}^{M}\right)$ satisfies (5.2) and (6.1) 
(with $n$ replaced by $n_{j}$ ), then any cluster point $u$ of $u_{n_{j}}$ in the weak topology of $W^{1, p}\left(\Omega^{\prime}, \mathbf{R}^{M}\right)$ satisfies (6.2). To simplify the notation, let us assume that the whole sequence $\left(u_{n_{j}}\right)$ converges weakly in $W^{1, p}\left(\Omega^{\prime}, \mathbf{R}^{M}\right)$ to $u$.

By Proposition 6.7, there exists a function $H \in L_{\mu}^{p^{\prime}}\left(\Omega^{\prime}, \mathbf{R}^{M}\right)$ such that $u$ satisfies (6.14). Estimate (6.28), applied with $u_{n}$ and $z_{n}$ replaced by $u_{n_{j}}$ and $q_{n_{j}}^{m}$, gives

$$
\left|H-H_{q}^{m}\right| \leq C\left(|u|+\left|q^{m}\right|\right)^{\frac{p-2}{p-1}}\left|u-q^{m}\right|^{\frac{1}{p-1}}, \quad \mu \text {-a.e. in } \Omega^{\prime},
$$

and therefore, taking the limit as $m$ tends to infinity we obtain

$$
|H-F(x, q w)| \leq C(|u|+|q w|)^{p \frac{p-2}{p-1}}|u-q w|^{\frac{1}{p-1}}, \quad \mu \text {-a.e. in } \Omega^{\prime},
$$

which implies that for any step function $\zeta=\sum_{i=i}^{m} q_{i} 1_{B_{i}}$, with $B_{i}$ Borel subset of $\Omega^{\prime}$ and $q_{i}$ in $\mathbf{Q}^{M}$, we get

$$
|H-F(x, \zeta w)| \leq C(|u|+|\zeta w|)^{p \frac{p-2}{p-1}}|u-\zeta w|^{\frac{1}{p-1}}, \quad \mu \text {-a.e. in } \Omega^{\prime} .
$$

Finally, Proposition 3.6 and the continuity property (III) of $F$ imply that $H(x)=$ $F(x, u(x)) \mu$-a.e. in $\Omega^{\prime}$, which concludes the proof.

7. Corrector. In this section, we shall fix the sequence $\left(\mu_{n}\right)$ in $\mathcal{M}_{0}^{p}(\Omega)$ and the sequence $\left(F_{n}\right)$ in $\mathcal{F}(L)$, with $L>0$, and we shall assume that $\left(\mu_{n}\right) \gamma^{-\Delta_{p_{-}}}$ converges to $\mu$ and the pairs $\left(\mu_{n}, F_{n}\right) \gamma^{A}$-converge to $(\mu, F)$, where $\mu \in \mathcal{M}_{0}^{p}(\Omega)$ and $F \in \mathcal{F}(\alpha, C, 1 /(p-1))$. This implies that in Theorem 6.9 the solutions $s_{n}^{m}$ of the problems (6.32) converge weakly in $W_{0}^{1, p}\left(\Omega, \mathbf{R}^{M}\right)$ to $s^{m}$ when $n$ tends to infinity without extracting any subsequence. Let us define $R_{n}^{m}: \Omega \times \mathbf{R}^{M} \mapsto \mathbf{M}^{M \times N}$ by

$$
R_{n}^{m}(x, s)=D s_{n}^{m}-D(s w) .
$$

The following result gives an approach in $L^{p}\left(\Omega, \mathbf{M}^{M \times N}\right)$ of the gradient of the solution $u_{n}$ of problem (5.2).

THEOREM 7.1. Let $\Omega^{\prime}$ be an open subset of $\Omega$. Let $\left(u_{n}\right)$ be a sequence, with $u_{n} \in$ $W^{1, p}\left(\Omega^{\prime}, \mathbf{R}^{M}\right) \cap L_{\mu_{n}}^{p}\left(\Omega^{\prime}, \mathbf{R}^{M}\right)$, which converges to a function $u$ weakly in $W^{1, p}\left(\Omega^{\prime}, \mathbf{R}^{M}\right)$ and satisfies (6.1). Suppose there exists a sequence $\left(f_{n}\right)$, with $f_{n} \in\left(W_{0}^{1, p}(\Omega) \cap\right.$ $\left.L_{\mu_{n}}^{p}(\Omega)\right)^{\prime}$, which converges to $f \in\left(W_{0}^{1, p}(\Omega) \cap L_{\mu_{n}}^{p}(\Omega)\right)^{\prime}$ in the sense of $\left(\mathcal{H}_{\Omega^{\prime}}\right)$ and such that $u_{n}$ satisfies problem (5.2).

Then, for every function $\zeta=\sum_{i=1}^{l} s_{i} 1_{K_{i}}$ with $s_{i}$ in $\mathbf{R}^{M}$ and $K_{i}$ closed subsets of $\Omega^{\prime}$ such that $w=0 \mu$-a.e. on $K_{i} \cap K_{j}$ for $i \neq j$, we have

$$
\begin{gathered}
\limsup _{m \rightarrow \infty} \lim _{n \rightarrow \infty} \int_{K}\left|D u_{n}-D u-R_{n}^{m}(x, \zeta)\right|^{p} d x \\
\leq C\left(\int_{K}|u|^{p} d \mu+\int_{K}|w \zeta|^{p} d \mu\right)^{\frac{p-2}{p-1}}\left(\int_{K}|u-w \zeta|^{p} d \mu\right)^{\frac{1}{p-1}},
\end{gathered}
$$

where $K=\bigcup_{i=i}^{l} K_{i}$ and $C$ is a positive constant which depends only on $\alpha, \beta$, and $L$.

Remark 7.2. The heuristic idea of Theorem 7.1 is to show that the sequence of the gradients of $u_{n}$ is, except for a sequence which converges strongly to zero in $L^{p}\left(\Omega, \mathbf{R}^{M}\right)$, equal to the gradient of $u$ plus a sequence of nonlinear functions of the variables $x$ and $u(x)$. This explains the nonlinearity of the function $F$. If it were possible to apply (7.2) by replacing $\zeta$ by $u / w$, we would get

$$
\lim _{k \rightarrow \infty} \limsup _{n \rightarrow \infty} \int_{\Omega}\left|D u_{n}-D u-R_{n}^{m}\left(x, \frac{u}{w}\right)\right|^{p} d x=0 .
$$


But the choice $\zeta=u / w$ in (7.2) is not possible since we do not know, a priori, if $R_{n}^{m}(x, s)$ is a Carathéodory function; so $R_{n}^{m}(x, u(x))$ may not even be measurable. We avoid this problem using the function $w \zeta$ to approach $u$. This approach is always possible by Proposition 3.6 part b.

Remark 7.3. When we consider $R_{n}^{m}(x, \zeta)$, the value of $\zeta$ on $K_{i} \cap K_{j}, i \neq j$, is not relevant. Indeed by taking in (7.2) $u_{n}=u=0$ (then $f_{n}=0$ ) and $\zeta=s 1_{K_{i} \cap K_{j}}$ we deduce

$$
\limsup _{m \rightarrow \infty} \limsup _{n \rightarrow \infty} \int_{K_{i} \cap K_{j}} R_{n}^{m}(x, s) d x=0 \quad \forall s \in \mathbf{R}^{M} .
$$

Remark 7.4. If $K$ is a compact subset of $\Omega^{\prime}$ such that $\mu(K)=0$, estimate (7.2) with $\zeta=0$ implies that $D u_{n}$ converges strongly to $D u$ in $L^{p}\left(K, \mathbf{R}^{M}\right)$.

Proof of Theorem 7.1. Let $s \in \mathbf{R}^{M}$ and let $K$ be a closed subset of $\Omega^{\prime}$. By Lemma 6.6 , for every $\varphi \in C_{c}^{\infty}\left(\Omega^{\prime}, \mathbf{R}^{M}\right)$, with $\varphi \geq 1_{K}$ in $\Omega^{\prime}$, we have

$$
\begin{gathered}
\limsup _{n \rightarrow \infty} \int_{K}\left|D\left(\left(u_{n}-s_{n}^{m}\right)-\left(u-s^{m}\right)\right)\right|^{p} d x \\
\leq C\left(\int_{\Omega^{\prime}}|u|^{p} \varphi d \mu+\int_{\Omega^{\prime}}\left|s^{m}\right|^{p} \varphi d \mu\right)^{\frac{p-2}{p-1}}\left(\int_{\Omega^{\prime}}\left|u-s^{m}\right|^{p} \varphi d \mu\right)^{\frac{1}{p-1}} .
\end{gathered}
$$

If $\varphi$ now decreases to $1_{K}$, by the fact that $\left(s^{m}\right)$ tends to $s w$ strongly in $W_{0}^{1, p}\left(\Omega, \mathbf{R}^{M}\right) \cap$ $L_{\mu}^{p}\left(\Omega, \mathbf{R}^{M}\right)$ and from (7.3) we deduce

$$
\begin{gathered}
\limsup _{m \rightarrow \infty} \limsup _{n \rightarrow \infty} \int_{K}\left|D\left(\left(u_{n}-s_{n}^{m}\right)-\left(u-s^{m}\right)\right)\right|^{p} d x \\
\leq C\left(\int_{K}|u|^{p} d \mu+\int_{K}|s w|^{p} d \mu\right)^{\frac{p-2}{p-1}}\left(\int_{K}|u-s w|^{p} d \mu\right)^{\frac{1}{p-1}} .
\end{gathered}
$$

Moreover, by inequality

$$
\left.|| \xi_{1}\right|^{p}-\left|\xi_{2}\right|^{p}\left|\leq p\left(\left|\xi_{1}\right|^{p-1}+\left|\xi_{2}\right|^{p-1}\right)\right| \xi_{1}-\xi_{2} \mid \quad \forall \xi_{1}, \xi_{2} \in \mathbf{M}^{M \times N},
$$

we get

$$
\begin{gathered}
\left.|| D\left(\left(u_{n}-s_{n}^{m}\right)-\left(u-s^{m}\right)\right)\right|^{p}-\left|D\left(\left(u_{n}-s_{n}^{m}\right)-(u-s w)\right)\right|^{p} \mid \\
\leq p\left(\left|D\left(\left(u_{n}-s_{n}^{m}\right)-\left(u-s^{m}\right)\right)\right|^{p-1}+\left|D\left(\left(u_{n}-s_{n}^{m}\right)-(u-s w)\right)\right|^{p-1}\right)\left|D\left(s^{m}-s w\right)\right|,
\end{gathered}
$$

and then by the strong convergence of $\left(s^{m}\right)$ in $W_{0}^{1, p}\left(\Omega, \mathbf{R}^{M}\right)$ we deduce

$\lim _{m \rightarrow \infty} \limsup _{n \rightarrow \infty} \int_{K}\left|D\left(\left(u_{n}-s_{n}^{m}\right)-\left(u-s^{m}\right)\right)\right|^{p} d x-\left|D\left(\left(u_{n}-s_{n}^{m}\right)-(u-s w)\right)\right|^{p} \mid d x=0$.

Thus by (7.4) and the definition of $R_{n}^{m}$, we get

$$
\begin{gathered}
\limsup _{m \rightarrow \infty} \limsup _{n \rightarrow \infty} \int_{K}\left|D\left(u_{n}-u-R_{n}^{m}(x, s)\right)\right|^{p} d x \\
\leq C\left(\int_{K}|u|^{p} d \mu+\int_{K}|s w|^{p} d \mu\right)^{\frac{p-2}{p-1}}\left(\int_{K}|u-s w|^{p} d \mu\right)^{\frac{1}{p-1}} .
\end{gathered}
$$


Consider now $\zeta=\sum_{i=1}^{l} s_{i} 1_{K_{i}}$, with $s_{i} \in \mathbf{R}^{M}$ and $K_{i}$ closed subsets of $\Omega^{\prime}$ such that $w=0 \mu$-a.e. on $K_{i} \cap K_{j}$, for $i \neq j$. By Lemma 4.5, we also have $|u|=0 \mu$-a.e. on $K_{i} \cap K_{j}$, for $i \neq j$. Then, if $K=\bigcup_{i=1}^{l} K_{i}$, by using (7.5) and Hölder's inequality we get

$$
\begin{gathered}
\limsup _{m \rightarrow \infty} \limsup _{n \rightarrow \infty} \int_{K}\left|D u_{n}-D u-R_{n}^{m}(x, \zeta)\right|^{p} d x \\
\leq \limsup _{m \rightarrow \infty} \limsup _{n \rightarrow \infty} \sum_{i=1}^{l} \int_{K_{i}}\left|D\left(u_{n}-u-R_{n}^{m}\left(x, s_{i}\right)\right)\right|^{p} d x \\
\leq C \sum_{i=1}^{l}\left(\int_{K_{i}}|u|^{p} d \mu+\int_{K_{i}}\left|w s_{i}\right|^{p} d \mu\right)^{\frac{p-2}{p-1}}\left(\int_{K_{i}}\left|u-w s_{i}\right|^{p} d \mu\right)^{\frac{1}{p-1}} \\
=C\left(\int_{K}|u|^{p} d \mu+\int_{K}|w \zeta|^{p} d \mu\right)^{\frac{p-2}{p-1}}\left(\int_{K}|u-w \zeta|^{p} d \mu\right)^{\frac{1}{p-1}}
\end{gathered}
$$

which concludes the proof.

8. Particular cases. In this section, we shall prove that some assumptions on the function $a$, as homogeneity or linearity, are inherited by function $F$. In [6] we construct an example which shows that the function $F$ in general can be nonlinear and nonhomogeneous.

Homogeneous case. Let $a$ be a function which satisfies conditions (i)-(v), as at the beginning of section 5 . Let us assume in addition that $a$ satisfies the following homogeneity condition:

(vi) for a.e. $x \in \Omega$, for every $t \in \mathbf{R}$, and for every $\xi \in \mathbf{M}^{M \times N}$,

$$
a(x, t \xi)=|t|^{p-2} t a(x, t \xi) .
$$

Moreover, let $\left(\mu_{n}\right)$ be a sequence in $\mathcal{M}_{0}^{p}(\Omega)$, and let $\left(F_{n}\right)$ be a sequence of functions in $\mathcal{F}(L)$ which satisfies the following condition:

(VII) for every $x \in \Omega$, for every $t \in \mathbf{R}$, and for every $s \in \mathbf{R}^{M}$,

$$
F_{n}(x, t s)=|t|^{p-2} t F_{n}(x, s) .
$$

Under these assumptions we have the following result.

THEOREM 8.1. If the function a satisfies conditions (i)-(vi) and the sequence $\left(F_{n}\right)$ satisfies conditions (I)-(VII), then in Theorem 6.4 the function $F$ can be chosen satisfying

$$
F(x, t s)=|t|^{p-2} t F(x, s)
$$

for every $x \in \Omega$, for every $t \in \mathbf{R}$, and for every $s \in \mathbf{R}^{M}$.

Proof. Assumptions (vi) and (VII) imply that for every $t \in \mathbf{R}$ and for every $q \in \mathbf{Q}^{M}$, the solution $q_{n}^{m}$ of (6.42) satisfies

$$
(t q)_{n}^{m}=t q_{n}^{m}, \quad \mu \text {-a.e. in } \Omega,
$$

where $(t q)_{n}^{m}$ is the solution of problem (6.42) with $q$ replaced by $t q$, which converges, according with Theorem 6.9 , to some function $(t q)^{m}$ weakly in $W_{0}^{1, p}\left(\Omega, \mathbf{R}^{M}\right)$ for every $m \in \mathbf{N}$. Then taking the limit as $n \rightarrow \infty$ we have

$$
(t q)^{m}=t q^{m}, \quad \mu \text {-a.e. in } \Omega .
$$


Therefore, the functions $H_{q}^{m}$ and $H_{t q}^{m}$ defined by (6.43) satisfy

$$
H_{t q}^{m}=t H_{q}^{m}, \quad \mu \text {-a.e. in } \Omega
$$

for every $t \in \mathbf{R}$ and for every $q \in \mathbf{Q}^{M}$. Thus, using that for every $q \in \mathbf{Q}^{M}$, the function $G(x, q)$ in the proof of Theorem 6.4 is defined as the limit in $m$ of $H_{q}^{m}$, the continuity of $G(x, s)$ with respect to the variable $s$ and that the function $F(x, s)$ satisfies (6.49), we conclude the proof.

In this special case we have the following result for the correctors defined by (7.1).

THEOREM 8.2. Assume that the function $a$ and the sequence $\left(F_{n}\right)$ satisfy, respectively, properties (vi) and (VII). Then, the function $R_{n}^{m}$ defined by (7.1) satisfies

$$
R_{n}^{m}(x, t s)=t R_{n}^{m}(x, s)
$$

for almost every $x \in \Omega$, for every $s \in \mathbf{R}^{M}$, and for every $t \in \mathbf{R}$.

Proof. Assumptions (vi) and (VII) imply that, for every $t \in \mathbf{R}$ and for every $s \in \mathbf{R}^{M},(t s)_{n}^{m}=t s_{n}^{m}$, where $s_{n}^{m}$ is the solution of (6.32) and $(t s)_{n}^{m}$ is the solution of problem (6.32) with $s$ replaced by $t s$. Thus the conclusion follows by the definition of $R_{n}^{m}$.

Linear case. Let us consider now the linear case, i.e., let us assume, with slight abuse of notation, that the function $a(x, \xi)$ is of the form $a(x) \xi$, where $a(x)$ is a measurable function from $\Omega$ on the linear applications from $\mathbf{M}^{M \times N}$ to $\mathbf{M}^{M \times N}$ which satisfies these hypotheses:

$\left(\mathrm{i}_{l}\right)$ there exists a constant $\alpha>0$ such that for every $\xi \in \mathbf{M}^{M \times N}$ and for a.e. $x \in \Omega$, we have

$$
a(x) \xi \xi \geq \alpha|\xi|^{2}
$$

(ii $l_{l}$ ) there exists a constant $\beta>0$ such that for every $\xi \in \mathbf{M}^{M \times N}$ and for a.e. $x \in \Omega$, we have

$$
|a(x) \xi| \leq \beta|\xi| .
$$

Remark 8.3. Hypotheses $\left(\mathrm{i}_{l}\right)$ and $\left(\mathrm{ii}_{l}\right)$ imply $(\mathrm{i})-(\mathrm{v})$ at the beginning of section 5 for $p=2$.

Let us denote by $\mathcal{F}_{l}(L)$, with $L>0$, the class of all vector functions from $\Omega \times \mathbf{R}^{M}$ to $\mathbf{R}^{M}$ which are linear in the second argument (i.e., of the form $\left.F(x) s\right)$ and which satisfy the following two conditions:

$\left(\mathrm{I}_{l}\right)$ for every $s \in \mathbf{R}^{M}$ and for every $x \in \Omega$ we have

$$
F(x) s s \geq \alpha|s|^{2} ;
$$

$\left(\mathrm{II}_{l}\right)$ for every $s \in \mathbf{R}^{M}$ and for every $x \in \Omega$ we have

$$
|F(x) s| \leq L|s| .
$$

Remark 8.4. It is easy to see that the class $\mathcal{F}_{l}(L)$ defined above is contained in the class $\mathcal{F}(L)$ defined in section 5 .

We are now in a position to state the following result.

Theorem 8.5. Assume that in Theorem $6.4, A u=-\operatorname{div}(a(x) D u)$, with a $(x)$ satisfying $\left(\mathrm{i}_{l}\right)$ and $\left(\mathrm{ii}_{l}\right)$, and that the sequence $\left(F_{n}\right)$ belongs to $\mathcal{F}_{l}(L)$. Then, the function $F$ which appears in the statement of Theorem 6.4 can be chosen in the class $\mathcal{F}_{l}\left(L^{\prime}\right)$, with $L^{\prime}>0$ different, in general, from $L$. 
Proof. We have already shown in Theorem 8.1 that $F$ is homogeneous in its second argument. The additivity of $F$ can be proved essentially with the same argument.

For the corrector result, as in section 7, let us assume that $\left(\mu_{n}\right) \gamma^{-\Delta_{\text {-converges }}}$ to $\mu$ and that the pairs $\left(\mu_{n}, F_{n}\right) \gamma^{A}$-converge to $(\mu, F)$ according with Definition 6.2 (where $A u=-\operatorname{div}(a(x) D u)$ ). In this case the function $R_{n}^{m}: \Omega \times \mathbf{R}^{M} \rightarrow \mathbf{M}^{M \times N}$ is given by $R_{n}^{m}(x, s)=D s_{n}^{m}(x)-D(w s)(x)$, where for every $s \in \mathbf{R}^{M}, s_{n}^{m}$ is the solution of the problem

$$
\left\{\begin{array}{l}
s_{n}^{m} \in H_{0}^{1}\left(\Omega, \mathbf{R}^{M}\right) \cap L_{\mu_{n}}^{2}\left(\Omega, \mathbf{R}^{M}\right), \\
\int_{\Omega} a(x) D s_{n}^{m} D v d x+\int_{\Omega} F_{n}(x) s_{n}^{m} v d \mu_{n}=m \int_{\Omega}\left(w_{n} s-s_{n}^{m}\right) v d x \\
\forall v \in H_{0}^{1}\left(\Omega, \mathbf{R}^{M}\right) \cap L_{\mu_{n}}^{2}\left(\Omega, \mathbf{R}^{M}\right) .
\end{array}\right.
$$

Clearly, the functions $R_{n}^{m}$ are linear in their second argument, and hence they are Carathéodory functions. This allows us to improve Theorem 7.1.

THEOREM 8.6. Let $\Omega^{\prime}$ be an open subset of $\Omega$. Let $\left(u_{n}\right)$, with $u_{n} \in H^{1}\left(\Omega^{\prime}, \mathbf{R}^{M}\right) \cap$ $L_{\mu_{n}}^{2}\left(\Omega^{\prime}, \mathbf{R}^{M}\right)$, be a sequence which converges weakly in $H^{1}\left(\Omega^{\prime}, \mathbf{R}^{M}\right)$ to some function $u$ and satisfies (6.1). Assume also that there exists a sequence $\left(f_{n}\right)$, with $f_{n}$ belonging to $\left(H_{0}^{1}\left(\Omega^{\prime}, \mathbf{R}^{M}\right) \cap L_{\mu_{n}}^{2}\left(\Omega^{\prime}, \mathbf{R}^{M}\right)\right)^{\prime}$, converging to some functional $f \in\left(H_{0}^{1}\left(\Omega, \mathbf{R}^{M}\right) \cap\right.$ $\left.L_{\mu}^{2}\left(\Omega, \mathbf{R}^{M}\right)\right)^{\prime}$ in the sense of $\left(\mathcal{H}_{\Omega^{\prime}}\right)$, such that $\left(u_{n}\right)$ satisfies the following problem:

$$
\left\{\begin{array}{l}
u_{n} \in H^{1}\left(\Omega^{\prime}, \mathbf{R}^{M}\right) \cap L_{\mu_{n}}^{2}\left(\Omega^{\prime}, \mathbf{R}^{M}\right), \\
\int_{\Omega} a(x) D u_{n} D v d x+\int_{\Omega} F_{n}(x) u_{n} v d \mu_{n}=\left\langle f_{n}, v\right\rangle \\
\forall v \in H_{0}^{1}\left(\Omega^{\prime}, \mathbf{R}^{M}\right) \cap L_{\mu_{n}}^{2}\left(\Omega^{\prime}, \mathbf{R}^{M}\right) .
\end{array}\right.
$$

Then, for every function $\psi \in H^{1}\left(\Omega^{\prime}, \mathbf{R}^{M}\right) \cap L^{\infty}\left(\Omega^{\prime}, \mathbf{R}^{M}\right)$ and for every closed set $K \subset \Omega$, we have

$$
\limsup _{m \rightarrow \infty} \limsup _{n \rightarrow \infty} \int_{K}\left|D u_{n}-D u-R_{n}^{m}(x) \psi\right|^{2} d x \leq C \int_{K}|u-w \psi|^{2} d \mu .
$$

In particular, if $u / w$ belongs to $L^{\infty}\left(K, \mathbf{R}^{M}\right)$, then we have

$$
\limsup _{m \rightarrow \infty} \limsup _{n \rightarrow \infty} \int_{K}\left|D u_{n}-D u-R_{n}^{m}(x) \frac{u}{w}\right|^{2} d x=0 .
$$

In order to prove Theorem 8.6, we need some preliminary lemmas.

Lemma 8.7. Let $W=\sup \left\{\left\|w_{n}\right\|_{L^{\infty}(\Omega)}\right\}$. Then for every $s \in \mathbf{R}^{M}$, the solutions $s_{n}^{m}$ of (8.2) satisfy

$$
\limsup _{m \rightarrow \infty} \limsup _{n \rightarrow \infty} \int_{\left\{\left|s_{n}^{m}\right| \geq 2^{k} W|s|\right\}}\left|D s_{n}^{m}\right|^{2} d x \leq C \frac{|s|^{2}}{k} \quad \forall k \in \mathbf{N} .
$$

Proof. For any $j \in \mathbf{N}$, let us consider the function $\Phi_{j}: \mathbf{R}^{M} \mapsto \mathbf{R}^{M}$ defined by

$$
\Phi_{j}(\zeta)= \begin{cases}0 & \text { if }|\zeta| \leq 2^{j-1} W|s|, \\ \frac{|\zeta|-2^{j-1} W|s|}{2^{j-1} W|s|} \zeta & \text { if } 2^{j-1} W|s|<|\zeta|<2^{j} W|s|, \\ \zeta & \text { if }|\zeta| \geq 2^{j} W|s| .\end{cases}
$$


Taking $\Phi_{j}\left(s_{n}^{m}\right)$ as a test function in (8.2), we get

$$
\int_{\Omega} a(x) D s_{n}^{m} D\left[\Phi_{j}\left(s_{n}^{m}\right)\right] d x+\int_{\Omega} F_{n}(x) s_{n}^{m} \Phi_{j}\left(s_{n}^{m}\right) d \mu_{n}+m \int_{\Omega}\left(s_{n}^{m}-w_{n} s\right) \Phi_{j}\left(s_{n}^{m}\right) d x=0,
$$

which implies

$$
\begin{gathered}
\alpha \int_{\left\{\left|s_{n}^{m}\right| \geq 2^{j} W|s|\right\}}\left|D s_{n}^{m}\right|^{2} d x \\
\leq-\int_{\left\{2^{j-1} W|s| \leq\left|s_{n}^{m}\right|<2^{j} W|s|\right\}} a(x) D s_{n}^{m} D\left[\Phi_{j}\left(s_{n}^{m}\right)\right] d x+m \int_{\Omega}\left|s_{n}^{m}-w_{n} s\right|\left|\Phi_{j}\left(s_{n}^{m}\right)\right| d x \\
\leq C \int_{\left\{2^{j-1} W|s| \leq\left|s_{n}^{m}\right|<2^{j} W|s|\right\}}\left|D s_{n}^{m}\right|^{2} d x+m \int_{\Omega}\left|s_{n}^{m}-w_{n} s\right|\left|\Phi_{1}\left(s_{n}^{m}\right)\right| d x,
\end{gathered}
$$

where we used that $\left|\Phi_{j}\left(s_{n}^{m}\right)\right| \leq\left|\Phi_{1}\left(s_{n}^{m}\right)\right|$ for every $j \in \mathbf{N}$, and the fact that, in the set $\left\{2^{j-1} W|s| \leq\left|s_{n}^{m}\right|<2^{j} W|s|\right\}$, we have $D\left[\Phi_{j}\left(s_{n}^{m}\right)\right]=D s_{n}^{m}\left(2\left|s_{n}^{m}\right|-2^{j-1} W|s|\right) / 2^{j-1} W|s|$, and hence $\left|D\left[\Phi_{j}\left(s_{n}^{m}\right)\right]\right| \leq 3\left|D s_{n}^{m}\right|$.

On the other hand, by (6.38) we can see that for every $k \in \mathbf{N}$ we have

$$
\sum_{j=1}^{k} \int_{\left\{2^{j-1} W|s| \leq\left|s_{n}^{m}\right|<2^{j} W|s|\right\}}\left|D s_{n}^{m}\right|^{2} d x \leq \int_{\Omega}\left|D s_{n}^{m}\right|^{2} d x \leq C|s|^{2}
$$

and therefore, for every $k \in \mathbf{N}$, there exists $j(k)$, with $1 \leq j(k) \leq k$, such that

$$
\int_{\left\{2^{j(k)-1} W|s| \leq\left|s_{n}^{m}\right|<2^{j(k)} W|s|\right\}}\left|D s_{n}^{m}\right|^{2} d x \leq C \frac{|s|^{2}}{k} .
$$

By (8.8), applied with $j(k)$, we deduce that for any $k \in \mathbf{N}$ we have

$$
\begin{aligned}
& \int_{\left\{\left|s_{n}^{m}\right| \geq 2^{k} W|s|\right\}}\left|D s_{n}^{m}\right|^{2} d x \\
& \quad \leq \int_{\left\{\left|s_{n}^{m}\right| \geq 2^{j(k)} W|s|\right\}}\left|D s_{n}^{m}\right|^{2} d x \leq C \frac{|s|^{2}}{k}+m \int_{\Omega}\left|s_{n}^{m}-w_{n} s \| \Phi_{1}\left(s_{n}^{m}\right)\right| d x,
\end{aligned}
$$

thus taking the limit as $n \rightarrow \infty$ and using that $\Phi_{1}(w s)=0$, we obtain

$$
\begin{gathered}
\limsup _{n \rightarrow \infty} \int_{\left\{\left|s_{n}^{m}\right| \geq 2^{k} W|s|\right\}}\left|D s_{n}^{m}\right|^{2} d x \leq C \frac{|s|^{2}}{k}+m \int_{\Omega}\left|s^{m}-w s\right|\left|\Phi_{1}\left(s^{m}\right)-\Phi_{1}(w s)\right| d x \\
\leq C \frac{|s|^{2}}{k}+C m \int_{\Omega}\left|s^{m}-w s\right|^{2} d x
\end{gathered}
$$

Since, by Theorem 6.9 , the second term on the right-hand side tends to zero when $m$ tends to infinity, estimate (8.6) is proved.

LEMmA 8.8. For every function $\varphi \in H_{0}^{1}(\Omega) \cap L^{\infty}(\Omega)$, with $\varphi \geq 0$, we have

$$
\limsup _{m \rightarrow \infty} \limsup _{n \rightarrow \infty} \int_{\Omega}\left|R_{n}^{m}(x)\right|^{2} \varphi d x \leq C \int_{\Omega} w^{2} \varphi d \mu .
$$

Proof. Let $s \in \mathbf{R}^{M}$, with $|s| \leq 1$. Let us define $\Psi_{k}: \mathbf{R}^{M} \mapsto \mathbf{R}^{M}$ by $\Psi_{k}(\zeta)=$ $\zeta-\Phi_{k}(\zeta)$, where $\Phi_{k}$ is the function defined by (8.7). Taking $\Psi_{k}\left(s_{n}^{m}-w_{n} s\right) \varphi$, with 
$\varphi \in H_{0}^{1}(\Omega) \cap L^{\infty}(\Omega)$ and $\varphi \geq 0$, as a test function in (8.2), we have

$$
\begin{aligned}
& \int_{\Omega} a(x) D s_{n}^{m} D\left[\Psi_{k}\left(s_{n}^{m}-w_{n} s\right)\right] \varphi d x+\int_{\Omega} a(x) D s_{n}^{m} \Psi_{k}\left(s_{n}^{m}-w_{n} s\right) \otimes D \varphi d x \\
+ & m \int_{\Omega}\left(s_{n}^{m}-w_{n} s\right) \Psi_{k}\left(s_{n}^{m}-w_{n} s\right) \varphi d x+\int_{\Omega} F_{n}(x) s_{n}^{m} \Psi_{k}\left(s_{n}^{m}-w_{n} s\right) \varphi d \mu_{n}=0,
\end{aligned}
$$

where the second term tends to zero when $n$ and then $m$ tend to infinity and where the third term in the left-hand side is positive. This permits us to write

$$
\begin{aligned}
& \alpha \int_{\left\{\left|s_{n}^{m}-w_{n} s\right| \leq 2^{k-1} W|s|\right\}}\left|D\left(s_{n}^{m}-w_{n} s\right)\right|^{2} \varphi d x+\int_{\Omega} F_{n}(x)\left(s_{n}^{m}-w_{n} s\right) \Psi_{k}\left(s_{n}^{m}-w_{n} s\right) \varphi d \mu_{n} \\
& \leq C \int_{\left\{2^{k-1} W|s|<\left|s_{n}^{m}-w_{n} s\right|<2^{k} W|s|\right\}}\left|D s_{n}^{m}\right|\left|D\left(s_{n}^{m}-w_{n} s\right)\right| \varphi d x \\
& +C \int_{\left\{\left|s_{n}^{m}-w_{n} s\right| \leq 2^{k-1} W|s|\right\}}\left|D\left(w_{n} s-w s\right)\right|\left|D\left(s_{n}^{m}-w_{n} s\right)\right| \varphi d x \\
& +C \int_{\left\{\left|s_{n}^{m}-w_{n} s\right| \leq 2^{k-1} W|s|\right\}}|D w s|\left|D\left(s_{n}^{m}-w_{n} s\right)\right| \varphi d x \\
& +C \int_{\Omega}\left|w_{n} s\right|\left|\Psi_{k}\left(s_{n}^{m}-w_{n} s\right)\right| \varphi d \mu_{n}+o_{m, n} .
\end{aligned}
$$

Since for $k \geq 2$

$$
\left|s_{n}^{m}\right| \geq\left|s_{n}^{m}-w_{n} s\right|-\left|w_{n} s\right| \geq\left|s_{n}^{m}-w_{n} s\right|-W|s| \geq 2^{k-2} W|s|
$$

in the set $\left\{\left|s_{n}^{m}-w_{n} s\right| \geq 2^{k-1} W|s|\right\}$, by Hölder's inequality, (6.38), and Lemma 8.7, we obtain

$$
\int_{\left\{2^{k-1} W|s|<\left|s_{n}^{m}-w_{n} s\right|<2^{k} W|s|\right\}}\left|D s_{n}^{m}\right|\left|D\left(s_{n}^{m}-w_{n} s\right)\right| \varphi d x \leq C \frac{\|\varphi\|_{L^{\infty}(\Omega)}}{\sqrt{k-2}}|s|^{2}+o_{m, n} .
$$

By the definition of $\Psi_{k}$ and $\left(\mathrm{I}_{l}\right)$, we have

$$
\begin{gathered}
\int_{\Omega} F_{n}(x)\left(s_{n}^{m}-w_{n} s\right) \Psi_{k}\left(s_{n}^{m}-w_{n} s\right) \varphi d \mu_{n} \geq \alpha \int_{\Omega}\left(s_{n}^{m}-w_{n} s\right) \Psi_{k}\left(s_{n}^{m}-w_{n} s\right) \varphi d \mu_{n} \\
\geq \alpha \int_{\Omega}\left|\Psi_{k}\left(s_{n}^{m}-w_{n} s\right)\right|^{2} \varphi d \mu_{n} .
\end{gathered}
$$

Therefore, using Young's inequality in (8.10) and taking into account that $|s| \leq 1$ and that the third term of the right-hand side of (8.10) tends to zero when $n$ and $m$ tend to infinity, we get

$$
\begin{gathered}
\int_{\left\{\left|s_{n}^{m}-w_{n} s\right| \leq 2^{k-1} W|s|\right\}}\left|D\left(s_{n}^{m}-w_{n} s\right)\right|^{2} \varphi d x+\int_{\Omega}\left|\Psi_{k}\left(s_{n}^{m}-w_{n} s\right)\right|^{2} \varphi d \mu_{n} \\
\leq C\left[\frac{\|\varphi\|_{L^{\infty}(\Omega)}}{\sqrt{k-2}}+\int_{\Omega}\left|D\left(w_{n} s-w s\right)\right|^{2} \varphi d x+\int_{\Omega}\left|w_{n} s\right|^{2} \varphi d \mu_{n}\right]+o_{m, n} \\
\leq C \frac{\|\varphi\|_{L^{\infty}(\Omega)}}{\sqrt{k-2}}+C \int_{\Omega} w^{2} \varphi d \mu+o_{m, n}
\end{gathered}
$$


where in the last inequality we used Lemma 4.2. Thus by (8.12), (8.11), and Lemmas 8.7 and 4.2 , we have

$$
\begin{gathered}
\int_{\Omega}\left|R_{n}^{m}(x) s\right|^{2} \varphi d x=\int_{\Omega}\left|D\left(s_{n}^{m}-w s\right)\right|^{2} \varphi d x \\
\leq 2 \int_{\left\{\left|s_{n}^{m}-w_{n} s\right| \leq 2^{k-1} W|s|\right\}}\left|D\left(s_{n}^{m}-w_{n} s\right)\right|^{2} \varphi d x+2 \int_{\left\{\left|s_{n}^{m}-w_{n} s\right| \leq 2^{k-1} W|s|\right\}}\left|D\left(w_{n} s-w s\right)\right|^{2} \varphi d x \\
+2 \int_{\left\{\left|s_{n}^{m}-w_{n} s\right|>2^{k-1} W|s|\right\}}\left|D s_{n}^{m}\right|^{2} \varphi d x+2 \int_{\left\{\left|s_{n}^{m}-w_{n} s\right|>2^{k-1} W|s|\right\}}|D(w s)|^{2} \varphi d x \\
\leq C\|\varphi\|_{L^{\infty}(\Omega)}\left(\frac{1}{\sqrt{k-2}}+\frac{1}{k-2}\right)+C \int_{\Omega} w^{2} \varphi d \mu+o_{m, n},
\end{gathered}
$$

which by the arbitrariness of $k$ implies

$$
\limsup _{m \rightarrow \infty} \limsup _{n \rightarrow \infty} \int_{\Omega}\left|R_{n}^{m}(x) s\right|^{2} \varphi d x \leq C \int_{\Omega} w^{2} \varphi d \mu .
$$

Since

$$
\left|R_{n}^{m}(x)\right|=\max \left\{\left|R_{n}^{m}(x) s\right|:|s| \leq 1\right\} \leq \sum_{i=1}^{N}\left|R_{n}^{m}(x) e_{i}\right|,
$$

where $\left\{e_{i}: i \leq i \leq N\right\}$ is the canonical basis of $\mathbf{R}^{N}$, Lemma 8.8 is proved.

Remark 8.9. If in Lemma 8.8, $\varphi$ belongs to $C_{c}^{\infty}(\Omega)$, then estimate (8.9) may be easily deduced from estimate (6.9) in Lemma 6.6. Remark also that Lemmas 8.7 and 8.8 can be easily generalized to the nonlinear case.

Proof of Theorem 8.6. By Lemma 8.8, for every closed $K \subset \Omega^{\prime}$ and for every function $\psi \in H^{1}\left(\Omega^{\prime}, \mathbf{R}^{M}\right)$, with $\psi \geq 0$, we have

$$
\limsup _{m \rightarrow \infty} \limsup _{n \rightarrow \infty} \int_{K}\left|R_{m}^{n}(x)\right|^{2} \psi d x \leq C \int_{K} w^{2} \psi d \mu .
$$

Indeed it is enough in (8.9) to take $\varphi$ equals to $\varphi_{n} \psi$, with $\varphi_{n} \in H_{0}^{1}\left(\Omega^{\prime}, \mathbf{R}^{M}\right) \cap$ $L^{\infty}\left(\Omega^{\prime}, \mathbf{R}^{M}\right)$ decreasing to the characteristic function of $K$.

Consider $\psi \in H^{1}\left(\Omega^{\prime}, \mathbf{R}^{M}\right) \cap L^{\infty}\left(\Omega^{\prime}, \mathbf{R}^{M}\right)$ and let $K$ be a closed subset of $\Omega^{\prime}$. By Theorem 7.1, for any function $\zeta=\sum_{i=1}^{l} s_{i} 1_{K_{i}}$, with $s_{i} \in \mathbf{R}$ and $K_{i}$ closed subsets of $\Omega^{\prime}$, such that $K=\bigcup_{i=1}^{l} K_{i}$ and $w=0 \mu$-a.e. on $K_{i} \cap K_{j}$, for $i \neq j$, we have

$$
\begin{gathered}
\int_{K}\left|D u_{n}-D u-R_{n}^{m}(x) \psi\right|^{2} d x \\
\leq 2 \int_{K}\left|D u_{n}-D u-R_{n}^{m}(x) \zeta\right|^{2} d x+2 \int_{K}\left|R_{n}^{m}(x)\right|^{2}|\psi-\zeta|^{2} d x \\
\leq C \int_{K}|u-w \zeta|^{2} d \mu+2 \sum_{i=1}^{l} \int_{K_{i}}\left|R_{n}^{m}(x)\right|^{2}\left|\psi-s_{i}\right|^{2} d x+o_{m, n} \\
\leq C \int_{K}|u-w \zeta|^{2} d \mu+C \sum_{i=1}^{l} \int_{K_{i}}\left|w \psi-s_{i} w\right|^{2} d \mu+o_{m, n} \\
=C \int_{K}|u-w \zeta|^{2} d \mu+C \int_{K}|w \psi-w \zeta|^{2} d \mu+o_{m, n},
\end{gathered}
$$


where we used (8.13). In order to obtain (8.4), it is enough to take $\zeta=\zeta_{k}$, where $\left(\zeta_{k}\right)$ is a sequence of step functions such that $\left(w \zeta_{k}\right)$ converges strongly to $w \psi$ in $L_{\mu}^{2}\left(\Omega^{\prime}, \mathbf{R}^{M}\right)$.

Assume now that $u / w$ belongs to $L^{\infty}\left(K, \mathbf{R}^{M}\right)$ and take $\varepsilon>0$. By estimates (8.4) and (8.13), we get

$$
\begin{gathered}
\int_{K}\left|D u_{n}-D u-R_{n}^{m}(x) \frac{u}{w}\right|^{2} d x \\
\leq \int_{K}\left|D u_{n}-D u-R_{n}^{m}(x) \frac{u}{w+\varepsilon}\right|^{2} d x+\int_{K}\left|R_{n}^{m}(x)\right|^{2}\left|\frac{u}{w+\varepsilon}-\frac{u}{w}\right|^{2} d x \\
\leq \int_{K}\left|u-\frac{w u}{w+\varepsilon}\right|^{2} d \mu+C \varepsilon^{2} \|\left.\frac{u}{w}\right|_{L^{\infty}\left(K, \mathbf{R}^{M}\right)} ^{2} \int_{K}\left|R_{n}^{m}(x)\right|^{2} \frac{1}{(w+\varepsilon)^{2}} d \mu+o_{m, n} \\
\leq \int_{K}\left|u-\frac{w u}{w+\varepsilon}\right|^{2} d \mu+C \varepsilon^{2}\left\|\frac{u}{w}\right\|_{L^{\infty}\left(K, \mathbf{R}^{M}\right)}^{2} \int_{K} \frac{w^{2}}{(w+\varepsilon)^{2}} d \mu+o_{m, n} .
\end{gathered}
$$

By using that $u$ belongs to $L_{\mu}^{2}\left(\Omega, \mathbf{R}^{M}\right)$ and the dominated convergence theorem, the first integral of the right-hand side of (8.15) tends to zero when $\varepsilon$ tends to zero. Since

$$
\frac{\varepsilon w}{(w+\varepsilon)^{2}}=\frac{\varepsilon}{w+\varepsilon} \frac{w}{w+\varepsilon} \leq 1,
$$

and hence by the fact that $\nu=w^{p-1} \mu$ is a Radon measure,

$$
\varepsilon \int_{K} \frac{w^{2}}{(w+\varepsilon)^{2}} d \mu \leq \int_{K} w d \mu<+\infty
$$

we get that the second integral on the right-hand side of (8.15) tends to zero when $\varepsilon$ tends to zero. We deduce (8.5) taking the limit in $n, m$, and then in $\varepsilon$.

9. Asymptotically equivalent operators. We saw in the previous sections that the properties of the function $F$ which appears in the limit problem (6.2) are strictly related to the properties of the function $a$ which define the differential operator $A$. The next proposition shows, in some sense, how the function $F$ depends on the behavior of $a(x, \xi)$ when $|\xi|$ is large.

Let $\tilde{a}: \Omega \times \mathbf{M}^{M \times N} \rightarrow \mathbf{M}^{M \times N}$ be a Carathéodory function which satisfies conditions (i)-(v), and suppose that the following property

$$
\lim _{|\xi| \rightarrow \infty} \frac{|a(x, \xi)-\tilde{a}(x, \xi)|}{|\xi|^{p-1}}=0
$$

holds uniformly with respect to $x$ in $\Omega$. Let $\tilde{A}$ be the differential operator given by $\tilde{A} u=-\operatorname{div}(\tilde{a}(x, D u))$.

Proposition 9.1. Suppose that the pair $\left(\mu_{n}, F_{n}\right)$, according to Definition 6.2, $\gamma^{A}$-converges to $(\mu, F)$.

If the functions $a$ and $\tilde{a}$ satisfy condition (9.1), then we also have that $\left(\mu_{n}, F_{n}\right)$ $\gamma^{\tilde{A}}$-converges to $(\mu, F)$.

Proof. According to the definition of the $\gamma^{\tilde{A}}$-convergence, we have to show that for any open subset $\Omega^{\prime}$ of $\Omega$, for any sequence of functionals $\left(f_{n}\right)$, with $f_{n} \in$ $\left(W_{0}^{1, p}\left(\Omega^{\prime}, \mathbf{R}^{M}\right) \cap L_{\mu_{n}}^{p}\left(\Omega^{\prime}, \mathbf{R}^{M}\right)\right)^{\prime}$, which converges to some $f \in\left(W_{0}^{1, p}\left(\Omega^{\prime}, \mathbf{R}^{M}\right) \cap\right.$ 
$\left.L_{\mu}^{p}\left(\Omega^{\prime}, \mathbf{R}^{M}\right)\right)^{\prime}$ in the sense of $\left(\mathcal{H}_{\Omega^{\prime}}\right)$, and for any sequence $\left(u_{n}\right)$ which satisfies $(6.1)$ and

$$
\left\{\begin{array}{l}
u_{n} \in W^{1, p}\left(\Omega^{\prime}, \mathbf{R}^{M}\right) \cap L_{\mu_{n}}^{p}\left(\Omega^{\prime}, \mathbf{R}^{M}\right), \\
\int_{\Omega^{\prime}} \tilde{a}\left(x, D u_{n}\right) D v d x+\int_{\Omega^{\prime}} F_{n}\left(x, u_{n}\right) v d \mu_{n}=\left\langle f_{n}, v\right\rangle \\
\forall v \in W_{0}^{1, p}\left(\Omega^{\prime}, \mathbf{R}^{M}\right) \cap L_{\mu_{n}}^{p}\left(\Omega^{\prime}, \mathbf{R}^{M}\right),
\end{array}\right.
$$

every cluster point of the sequence $\left(u_{n}\right)$ in the weak topology of $W^{1, p}\left(\Omega^{\prime}, \mathbf{R}^{M}\right)$ satisfies problem

$$
\left\{\begin{array}{l}
u \in W^{1, p}\left(\Omega^{\prime}, \mathbf{R}^{M}\right) \cap L_{\mu}^{p}\left(\Omega^{\prime}, \mathbf{R}^{M}\right), \\
\int_{\Omega^{\prime}} \tilde{a}(x, D u) D v d x+\int_{\Omega^{\prime}} F(x, u) v d \mu=\langle f, v\rangle \\
\forall v \in W_{0}^{1, p}\left(\Omega^{\prime}, \mathbf{R}^{M}\right) \cap L_{\mu}^{p}\left(\Omega^{\prime}, \mathbf{R}^{M}\right) .
\end{array}\right.
$$

If $u_{n}$ satisfies (9.2), then it also satisfies

$$
\left\{\begin{array}{l}
u_{n} \in W^{1, p}\left(\Omega^{\prime}, \mathbf{R}^{M}\right) \cap L_{\mu_{n}}^{p}\left(\Omega^{\prime}, \mathbf{R}^{M}\right), \\
\int_{\Omega^{\prime}} a\left(x, D u_{n}\right) D v d x+\int_{\Omega^{\prime}} F_{n}\left(x, u_{n}\right) v d \mu_{n}=\left\langle g_{n}, v\right\rangle \\
\forall v \in W_{0}^{1, p}\left(\Omega^{\prime}, \mathbf{R}^{M}\right) \cap L_{\mu_{n}}^{p}\left(\Omega^{\prime}, \mathbf{R}^{M}\right),
\end{array}\right.
$$

where $g_{n}=f_{n}-\operatorname{div}\left[a\left(x, D u_{n}\right)-\tilde{a}\left(x, D u_{n}\right)\right]$. Therefore, once we show that $\left(\operatorname{div}\left[a\left(x, D u_{n}\right)-\right.\right.$ $\left.\left.\tilde{a}\left(x, D u_{n}\right)\right]\right)$ converges in the sense of $\left(\mathcal{H}_{\Omega^{\prime}}\right)$ to $\operatorname{div}[a(x, D u)-\tilde{a}(x, D u)]$, by the $\gamma^{A}$ convergence of $\left(\mu_{n}, F_{n}\right)$ to $(\mu, F)$, we can deduce that $u$ satisfies $(9.3)$.

In order to prove that $\left(-\operatorname{div}\left[\tilde{a}\left(x, D u_{n}\right)-a\left(x, D u_{n}\right)\right]\right)$ converges in the sense of $\left(\mathcal{H}_{\Omega^{\prime}}\right)$, let us consider $v_{n} \in W_{0}^{1, p}\left(\Omega^{\prime}, \mathbf{R}^{M}\right) \cap L_{\mu_{n}}^{p}\left(\Omega^{\prime}, \mathbf{R}^{M}\right)$ such that $\left(v_{n}\right)$ converges weakly to some $v$ in $W_{0}^{1, p}\left(\Omega^{\prime}, \mathbf{R}^{M}\right)$. Since by Proposition 5.4 the sequence $\left(D u_{n}\right)$ converges to $D u$ pointwise a.e. in $\Omega^{\prime}$, by Egorov's theorem, for every $\delta>0$, there exists a set $E \subseteq \Omega^{\prime}$, with $|E|<\delta$, such that $\left(D u_{n}\right)$ converges uniformly to $D u$ in $\Omega^{\prime} \backslash E$. Thus we get

$$
\begin{gathered}
\lim _{n \rightarrow \infty} \int_{\Omega^{\prime}}\left[\tilde{a}\left(x, D u_{n}\right)-a\left(x, D u_{n}\right)\right] D v_{n} d x \\
=\int_{\Omega^{\prime} \backslash E}[\tilde{a}(x, D u)-a(x, D u)] D v d x+\lim _{n \rightarrow \infty} \int_{E}\left[\tilde{a}\left(x, D u_{n}\right)-a\left(x, D u_{n}\right)\right] D v_{n} d x
\end{gathered}
$$

Let us estimate the last limit in (9.4). By (9.1), for every $\varepsilon>0$ there exists $M>0$ such that

$$
|\tilde{a}(x, \xi)-a(x, \xi)| \leq \varepsilon|\xi|^{p-1}
$$

whenever $|\xi|>M$. Thus, since $\left(v_{n}\right)$ and $\left(u_{n}\right)$ are bounded in $W^{1, p}\left(\Omega^{\prime}, \mathbf{R}^{M}\right)$, by 
Hölder's inequality, we get

$$
\begin{gathered}
\lim _{n \rightarrow \infty}\left|\int_{E}\left[\tilde{a}\left(x, D u_{n}\right)-a\left(x, D u_{n}\right)\right] D v_{n} d x\right| \\
\leq \lim _{n \rightarrow \infty} \int_{E \cap\left\{\left|D u_{n}\right|>M\right\}_{\Omega^{\prime}}}\left|\tilde{a}\left(x, D u_{n}\right)-a\left(x, D u_{n}\right)\right|\left|D v_{n}\right| d x \\
+\int_{E \cap\left\{\left|D u_{n}\right| \leq M\right\}_{\Omega^{\prime}}}\left|\tilde{a}\left(x, D u_{n}\right)-a\left(x, D u_{n}\right)\right|\left|D v_{n}\right| d x \leq C\left(\varepsilon+M^{p-1} \delta^{(p-1) / p}\right) .
\end{gathered}
$$

Now taking the limit as $\delta$ goes to zero and then the limit as $M$ goes to infinity, by (9.4) and (9.5) we obtain

$$
\lim _{n \rightarrow \infty} \int_{\Omega^{\prime}}\left[\tilde{a}\left(x, D u_{n}\right)-a\left(x, D u_{n}\right)\right] D v_{n} d x=\int_{\Omega^{\prime}}[\tilde{a}(x, D u)-a(x, D u)] D v d x,
$$

which concludes the proof.

COROLlaRY 9.2. Let $\left(F_{n}\right)$ be a sequence in $\mathcal{F}(L)$ which satisfies condition (VI) and assume that the function a satisfies the following condition: There exists a Carathéodory function ã such that

$$
\lim _{t \rightarrow \infty} \frac{a(x, t \xi)}{|t|^{p-2} t}=\tilde{a}(x, \xi)
$$

uniformly in $x$, for every $\xi \in \mathbf{M}^{M \times N}$.

Suppose that the pair $\left(\mu_{n}, F_{n}\right)$, according to Definition $6.2, \gamma^{A}$-converges to $(\mu, F)$. Then the function $F$ also satisfies condition (VI).

Proof. It is easy to see that $\tilde{a}$ satisfies conditions (i)-(vi) and that condition (9.6) implies condition (9.1). Thus by the previous theorem the sequence of pairs $\left(F_{n}, \mu_{n}\right) \gamma^{\tilde{A}}$-converges to $(\mu, F)$ and by Theorem 8.1 the function $F$ satisfies condition (VI).

10. General operators. In this section we shall prove that the results given in the previous sections hold for a class of more general operators. Actually, let $2 \leq p<+\infty$ and let $b: \Omega \times \mathbf{R}^{M} \times \mathbf{M}^{M \times N} \mapsto \mathbf{M}^{M \times N}$ be a Carathéodory function such that:

(i') there exists a constant $\alpha>0$ such that

$$
\left(b\left(x, 0, \xi_{1}\right)-b\left(x, 0, \xi_{2}\right)\right)\left(\xi_{1}-\xi_{2}\right) \geq \alpha\left|\xi_{1}-\xi_{2}\right|^{p}
$$

for every $s \in \mathbf{R}^{M}$, for every $\xi_{1}, \xi_{2} \in \mathbf{M}^{M \times N}$, and for a.e. $x \in \Omega$;

(ii') there exists a constant $\beta>0$ and a function $h \in L^{\frac{p}{p-2}}(\Omega)(p /(p-2)=+\infty$ if $p=2$ ) such that

$$
\left|b\left(x, 0, \xi_{1}\right)-b\left(x, 0, \xi_{2}\right)\right| \leq \beta\left(h(x)+\left(\left|\xi_{1}\right|+\left|\xi_{2}\right|\right)^{p-2}\right)\left|\xi_{1}-\xi_{2}\right|
$$

for every $\xi_{1}, \xi_{2} \in \mathbf{M}^{M \times N}$ and for a.e. $x \in \Omega$;

(iii') there exists a constant $\gamma>0$ and a function $k \in L^{p^{\prime}}(\Omega)$ such that

$$
\left|b\left(x, s_{1}, \xi\right)-b\left(x, s_{2}, \xi\right)\right| \leq \gamma\left(k(x)+\left(\left|s_{1}\right|+\left|s_{2}\right|\right)^{q}+|\xi|^{r}\right) \min \left\{\left|s_{1}-s_{2}\right|, 1\right\}
$$

for every $s_{1}, s_{2} \in \mathbf{R}^{M}$, for every $\xi \in \mathbf{M}^{M \times N}$ and for a.e. $x \in \Omega$, where $q$ and $r$ are constants which satisfy $0 \leq q<N(p-1) /(N-p)$ if $p<N, q \geq 0$ if $p \geq N$ and $0 \leq r<p-1$. 
$\left(\mathrm{iv}^{\prime}\right) b(\cdot, 0,0) \in L^{p^{\prime}}(\Omega)$.

Under these hypotheses on the operator $B u=-\operatorname{div}(b(x, u, D u))$, we have the following generalizations of Definition 6.2 and Theorem 6.4.

Definition 10.1. Let $\left(\mu_{n}\right)$ be a sequence in $\mathcal{M}_{0}^{p}(\Omega)$, let $\left(F_{n}\right)$ be a sequence in $\mathcal{F}\left(c_{1}, c_{2}, \sigma\right)$, let $\mu \in \mathcal{M}_{0}^{p}(\Omega)$ and $F \in \mathcal{F}\left(c_{1}, c_{2}, \sigma\right)$. We say that the pairs $\left(\mu_{n}, F_{n}\right) \gamma^{B}$ converge (in $\Omega$ ) to the pair $(\mu, F)$ if the following property holds: for any open set $\Omega^{\prime} \subseteq$ $\Omega$, for any sequence of functionals $\left(f_{n}\right)$, with $f_{n} \in\left(W_{0}^{1, p}\left(\Omega^{\prime}, \mathbf{R}^{M}\right) \cap L_{\mu_{n}}^{p}\left(\Omega^{\prime}, \mathbf{R}^{M}\right)\right)^{\prime}$, which converges to some $f \in\left(W_{0}^{1, p}\left(\Omega^{\prime}, \mathbf{R}^{M}\right) \cap L_{\mu}^{p}\left(\Omega^{\prime}, \mathbf{R}^{M}\right)\right)^{\prime}$ in the sense of $\left(\mathcal{H}_{\Omega^{\prime}}\right)$ (according to Definition 5.1), and for any sequence $\left(u_{n}\right)$ of solutions of the problems

$$
\left\{\begin{array}{l}
u_{n} \in W^{1, p}\left(\Omega^{\prime}, \mathbf{R}^{M}\right) \cap L_{\mu_{n}}^{p}\left(\Omega^{\prime}, \mathbf{R}^{M}\right), \\
\int_{\Omega^{\prime}} b\left(x, u_{n}, D u_{n}\right) D v d x+\int_{\Omega^{\prime}} F_{n}\left(x, u_{n}\right) v d \mu_{n}=\left\langle f_{n}, v\right\rangle \\
\forall v \in W_{0}^{1, p}\left(\Omega^{\prime}, \mathbf{R}^{M}\right) \cap L_{\mu_{n}}^{p}\left(\Omega^{\prime}, \mathbf{R}^{M}\right)
\end{array}\right.
$$

satisfying (6.1), all cluster points of the sequence $\left(u_{n}\right)$ in the weak topology of $W^{1, p}\left(\Omega^{\prime}, \mathbf{R}^{M}\right)$ satisfy the following problem:

$$
\left\{\begin{array}{l}
u \in W^{1, p}\left(\Omega^{\prime}, \mathbf{R}^{M}\right) \cap L_{\mu}^{p}\left(\Omega^{\prime}, \mathbf{R}^{M}\right), \\
\int_{\Omega^{\prime}} b(x, u, D u) D v d x+\int_{\Omega^{\prime}} F(x, u) v d \mu=\langle f, v\rangle \\
\forall v \in W_{0}^{1, p}\left(\Omega^{\prime}, \mathbf{R}^{M}\right) \cap L_{\mu}^{p}\left(\Omega^{\prime}, \mathbf{R}^{M}\right) .
\end{array}\right.
$$

Remark 10.2. If $\left(u_{n}\right)$ is a sequence of solutions of problems (10.1) then the assertion of Proposition 5.4 can be proved using the same argument.

THEOREM 10.3. Let $\left(\mu_{n}\right)$ be a sequence of measures in $\mathcal{M}_{0}^{p}(\Omega)$ and let $\left(F_{n}\right)$ be a sequence in $\mathcal{F}(L)$, with $L>0$. Then there exists an increasing sequence of integers $\left(n_{j}\right)$, a measure $\mu \in \mathcal{M}_{0}^{p}(\Omega)$, and a function $F \in \mathcal{F}(\alpha, C, 1 /(p-1))$ such that the pairs $\left(\mu_{n_{j}}, F_{n_{j}}\right) \gamma^{B}$-converge to $(\mu, F)$ in $\Omega$ (according to Definition 10.1).

Proof. The above hypotheses on $b(x, s, \xi)$ imply that the application $a: \Omega \times$ $\mathbf{M}^{M \times N} \mapsto \mathbf{M}^{M \times N}$ defined by $a(x, \xi)=b(x, 0, \xi)-b(x, 0,0)$ satisfies conditions (i)-(v) in section 5 and then, by Theorem 6.4, there exists an increasing sequence of integers $\left(n_{j}\right)$, a measure $\mu \in \mathcal{M}_{0}^{p}(\Omega)$, and a function $F \in \mathcal{F}(\alpha, C, 1 /(p-1))$ such that the pairs $\left(\mu_{n_{j}}, F_{n_{j}}\right) \gamma^{A}$-converge to $(\mu, F)$ in $\Omega$ (according to Definition 6.2). Let us see that the pairs $\left(\mu_{n_{j}}, F_{n_{j}}\right) \gamma^{B}$-converge to $(\mu, F)$ in $\Omega$ (according to Definition 10.1). Let us consider a sequence of functionals $\left(f_{n_{j}}\right)$, with $f_{n_{j}} \in\left(W_{0}^{1, p}\left(\Omega^{\prime}, \mathbf{R}^{M}\right) \cap L_{\mu_{n_{j}}}^{p}\left(\Omega^{\prime}, \mathbf{R}^{M}\right)\right)^{\prime}$, which converges to some $f \in\left(W_{0}^{1, p}\left(\Omega^{\prime}, \mathbf{R}^{M}\right) \cap L_{\mu}^{p}\left(\Omega^{\prime}, \mathbf{R}^{M}\right)\right)^{\prime}$ in the sense of $\left(\mathcal{H}_{\Omega^{\prime}}\right)$, a sequence $\left(u_{n_{j}}\right)$ which satisfies (10.1) (with $n$ replaced by $n_{j}$ ) and (6.1), and a cluster point $u$ of the sequence $\left(u_{n_{j}}\right)$ in the weak topology of $W^{1, p}\left(\Omega^{\prime}, \mathbf{R}^{M}\right)$. We have to prove that $u$ satisfies problem (10.2). In order to simplify the notation, we shall still denote by $\left(u_{n_{j}}\right)$ the subsequence of $\left(u_{n_{j}}\right)$ which converges weakly in $W^{1, p}\left(\Omega^{\prime}, \mathbf{R}^{M}\right)$ to $u$. By (10.1), the sequence $\left(u_{n_{j}}\right)$ satisfies

$$
\left\{\begin{array}{l}
u_{n_{j}} \in W^{1, p}\left(\Omega^{\prime}, \mathbf{R}^{M}\right) \cap L_{\mu_{n_{j}}}^{p}\left(\Omega^{\prime}, \mathbf{R}^{M}\right), \\
\int_{\Omega^{\prime}} a\left(x, D u_{n_{j}}\right) D v d x+\int_{\Omega^{\prime}} F\left(x, u_{n_{j}}\right) v d \mu=\left\langle f_{n_{j}}, v\right\rangle-\left\langle g_{n_{j}}, v\right\rangle \\
\forall v \in W_{0}^{1, p}\left(\Omega^{\prime}, \mathbf{R}^{M}\right) \cap L_{\mu_{n_{j}}}^{p}\left(\Omega^{\prime}, \mathbf{R}^{M}\right),
\end{array}\right.
$$


where $g_{n_{j}}=-\operatorname{div}\left(b\left(x, u_{n_{j}}, D u_{n_{j}}\right)-b\left(x, 0, D u_{n_{j}}\right)-b(x, 0,0)\right)$. To conclude the proof it is enough to show that the sequence $\left(g_{n_{j}}\right)$ converges in the sense of $\left(\mathcal{H}_{\Omega^{\prime}}\right)$ to the functional $g=-\operatorname{div}(b(x, u, D u)-b(x, 0, D u)-b(x, 0,0))$. By (iii') we have

$$
\left|b\left(x, u_{n_{j}}, D u_{n_{j}}\right)-b\left(x, 0, D u_{n_{j}}\right)\right| \leq \gamma\left(k+\left|u_{n_{j}}\right|^{q}+\left|D u_{n_{j}}\right|^{r}\right)\left|u_{n_{j}}\right| .
$$

By Remark 10.2, $D u_{n_{j}}$ converges pointwise to $D u$, and then the left-hand side of (10.4) converges pointwise to $b(x, u, D u)-b(x, 0, D u)$, and the power $p^{\prime}$ of the right-hand side is uniformly integrable. This implies that $\left(b\left(x, u_{n_{j}}, D u_{n_{j}}\right)-b\left(x, 0, D u_{n_{j}}\right)\right)$ converges strongly in $L^{p^{\prime}}\left(\Omega^{\prime}\right)$ to $b(x, u, D u)-b(x, 0, D u)$, which concludes the proof.

\section{REFERENCES}

[1] L. Boccardo And F. Murat, Almost everywhere convergence of the gradients of solutions to elliptic and parabolic equations, Nonlinear Anal., 19 (1992), pp. 581-597.

[2] G. Buttazzo and G. Dal Maso, Shape optimization for Dirichlet problems: Relaxed solutions and optimality conditions, Appl. Math. Optim., 23 (1991), pp. 17-49.

[3] J. Casado-Diaz, Homogenization of Dirichlet problems for monotone operators in varying domains, Proc. Roy. Soc. Edinburgh Sect. A, 127 (1997), pp. 457-478.

[4] J. CASADO-Diaz, Existence of a sequence satisfying Cioranescu-Murat conditions in homogenization of Dirichlet problems in perforated domains, Rend. Mat. Appl. (7), 16 (1996), pp. 387-413.

[5] J. Casado-Diaz, Homogenization of pseudomonotone Dirichlet problems in varying domains, J. Math. Pures Appl., to appear.

[6] J. Casado-Diaz and A. Garroni, A non homogeneous extra term for the limit of Dirichlet problems in perforated domains, in Homogenization and Applications to Material Sciences, Math. Sciences and Appl. Series, Gakkokotosho, 1995.

[7] D. Cioranescu and F. Murat, Un Term Étrange Venu D'Ailleurs, in Nonlinear Partial Differential Equations and Their Applications, Collège de France seminar, Vol. II and III, H. Brézis and J.-L. Lions, eds., Research Notes in Math. 60 and 70, Pitman, London, 1982, pp. $98-138$ and pp. $154-178$.

[8] G. Dal Maso, On the integral representation of certain local functionals, Ricerche Mat., 32 (1983), pp. 85-113.

[9] G. Dal Maso, An Introduction to Г-Convergence, Birkhäuser, Boston, MA, 1993.

[10] G. Dal Maso And A. Defranceschi, Limits of nonlinear Dirichlet problems in varying domains, Manuscripta Math., 61 (1988), pp. 251-278.

[11] G. Dal Maso and A. Garroni, New results on the asymptotic behaviour of Dirichlet problems in perforated domains, Math. Models Methods Appl. Sci., 3 (1994), pp. 373-407.

[12] G. Dal Maso, A. Garroni, and I. V. Skrypnik, A capacitary method for the asymptotic analysis of Dirichlet problems for monotone operators, J. Anal. Math., 71 (1997), pp. $263-313$.

[13] G. Dal Maso and U. Mosco, Wiener-criterion and $\Gamma$-convergence, Appl. Math. Optim., 15 (1987), pp. 15-63.

[14] G. Dal Maso and U. Mosco, Wiener-criteria and energy decay for relaxed Dirichlet problems, Arch. Rational Mech. Anal., 95 (1986), pp. 345-387.

[15] G. Dal Maso and F. Murat, Asymptotic behaviour and correctors for Dirichlet problems in perforated domains with homogeneous monotone operators, Ann. Scuola Norm. Sup. Pisa Cl. Sci. (4), 24 (1997), pp. 239-290.

[16] G. Dal Maso and F. Murat, Dirichlet problems in perforated domains for homogeneous monotone operators on $H_{0}^{1}$, in Calculus of Variations, Homogenization and Continuum Mechanics, G. Bouchitté, G. Buttazzo, and P. Suquet, eds., Series Adv. Math. Appl. Sci. 18, World Scientific, Singapore, 1994, pp. 177-202.

[17] G. Dal Maso and I. V. Skrypnik, Capacitary theory for monotone operators, Potential Anal., 7 (1997), pp. 765-803.

[18] A. Defranceschi And E. Vitali, Limits of minimum problems with convex obstacles for vector valued functions, Appl. Anal., 52 (1994), pp. 1-33.

[19] L. C. Evans and R. F. Gariepy, Measure Theory and Fine Properties of Functions, Studies in Advanced Mathematics, CRC Press, Boca Raton, FL, 1992.

[20] E. YA. Khruslov, The method of orthogonal projections and the Dirichlet problems in domains with a fine-grained boundary, Math. USSR-Sb., 17 (1972), pp. 37-59. 
[21] N. Labani And C. Picard, Homogenization of a nonlinear Dirichlet problem in a periodically perforated domain, in Recent Advances in Nonlinear Elliptic and Parabolic Problems, P. Bénilan, M. Chipot, L. C. Evans, and M. Pierre, eds., Pitman Res. Notes Math. Ser. 208, Longman, Harlow, 1989, pp. 294-305.

[22] A. V. Marchenko and E. Ya. Khruslov, Boundary Value Problems in Domains with FineGranulated Boundaries, Naukova Dumka, Kiev, 1974 (in Russian).

[23] J. L. Lions, Quelques Méthodes de résolution des Problémes aux Limites Non Linéaires, Dunod, Gauthier-Villars, Paris, 1969.

[24] I. V. Skrypnik, Nonlinear Elliptic Boundary Value Problems, Teubner-Verlag, Leipzig, 1986.

[25] I. V. SKRYPNIK, Asymptotic behaviour of solutions of nonlinear elliptic problems in perforated domains, Math. Sbornik, 184, 10 (1993), pp. 67-90.

[26] I. V. SkRYPNik, Homogenization of nonlinear Dirichlet problems in perforated domains of general structure, Mat. Sb. (N.S.), to appear.

[27] I. V. SKRYPNiK, New conditions for the homogenization of nonlinear Dirichlet problems in perforated domains, Ukraïn. Mat. Zh., 48 (1996), pp. 675-694.

[28] W. P. Ziemer, Weakly Differentiable Functions, Springer-Verlag, Berlin, 1989. 